Universidade de São Paulo

Instituto de Física e Química de São Carlos

Tratamento Geral de Sistemas

Coulombianos de três Corpos pelo Formalismo Hiperesférico

Jean-Jacques G. S. De Groote

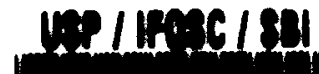 \\ 8.26Titit

Tese apresentada ao Instituto de

Física e Química de São Carlos para obtenção do título de Doutor em Física Básica.

Orientador: Prof. Dr. José Eduardo Martinho Hornos

Departamento de Física e Ciência dos Materiais São Carlos - 1993

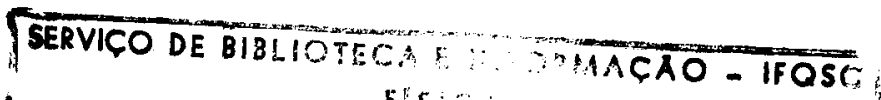




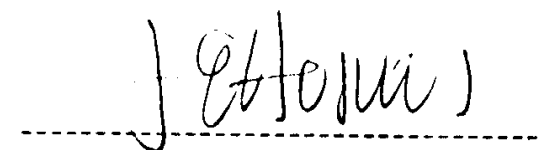

Prof.Dr. Jose Eduardo Hartinho Hornos

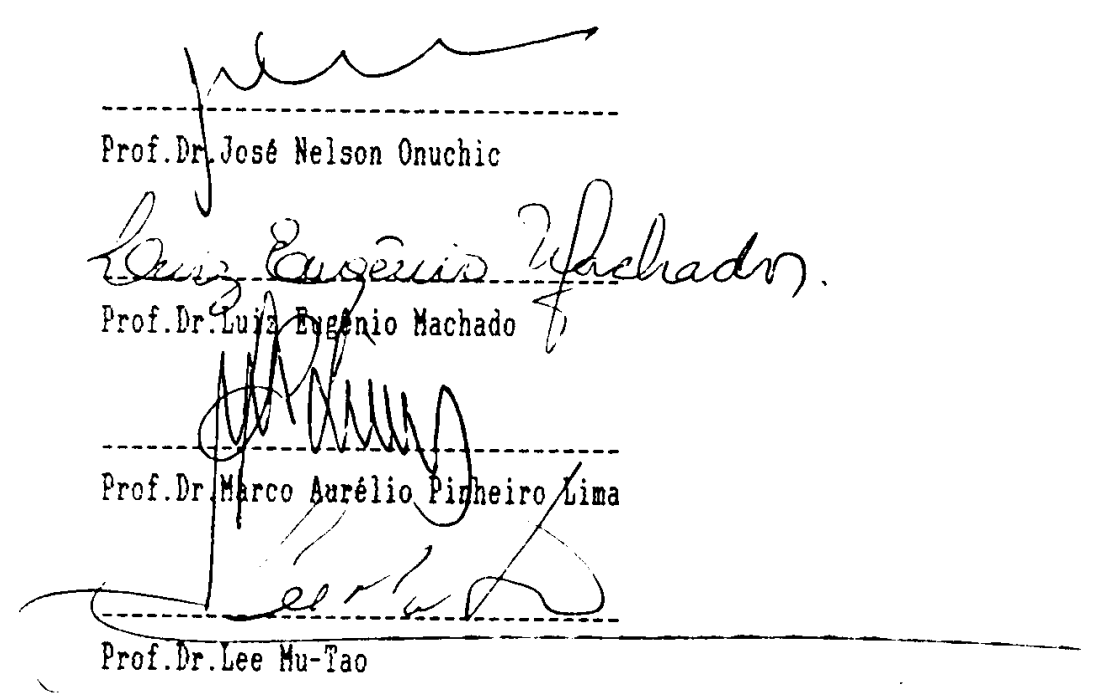


À meu pai, Robert, por tudo. 
à minha mãe Angela e meus irmãos, Michel, Roland, Francine e Suzanne. 
Este trabalho foi realizado com o apoio financeiro do CNPQ. 


\section{Agradecimentos}

A execução e conclusão deste trabalho só foram possíveis graças a ajuda direta ou indireta de muitas pessoas, algumas das quais cito abaixo como uma pequena forma de reconhecimento. Gostaria de agradeçer às seguintes pessoas,

- ao meu orientador, José Eduardo Hornos, responsável por minha formação científica. Sua amizade e dedicaşão incentivaram o desenvolvimento de meu traballıo, e foram fundamentais para sua conclusão, agradeço também a sua esposa, Yvone por todo apoio,

- aos meus amigos Mauro, Esmerindo, Ricardo, Alexandre, membros de meu grupo de trabalho pelo apoio e presença em qualquer dificuldade,

- aos funcionários do Instituto, em especial Irene, Marcia, Wladerez, pela ajuda e paciência,

- aos meus amigos da pós-graduação e graduação, entre eles Dani, Washington, Zé Paulo, Fernando, Gabriel, Facin, Jeremias, Vitor, Ubiraci, Salviano, Alexandre, Virgílio, Marcio, Diogenes, que tornam o ambiente de trabalho um lugar especial,

- ao Prof. H.T.Coelho, pela importante participação na discussão e publicação dos resultados obtidos,

- aos colegas de minha turma e grandes amigos, Aparecida, Marcel, Liliane, Reginaldo, Milled, Hemrique, Sergio, com quem compartilhei muitos anos de faculdade,

- aos membros da banca, José N. Onuchic, Marco A. P. Lima, Lee Mutao e Luiz E. Machado pela crítica construtiva ao trabalho e pelo incentivo e sugestões a sua continuação,

- e principalmente à minlıa esposa, Karina, por seu carinho e ajuda em todas as fases deste trabalho, me forneçendo um apoio que não posso expressar em palavras e certamente não poderia passar sem ele. 


\section{N D I C E}

RESUMO

ABSTRACT

CAPÍTULO 1: INTRODUÇÃ̃o 3

CAPÍTULO 2: O MÉTODO HIPERESFÉrICO ADIABÁTICO 11

2.1 - Fomalismo Hiperesférico 12

2.2 - Procedimento Hiperesférico Adiabático 14

$\begin{array}{ll}2.3 \text { - Propriedades da Equação angular } & 16\end{array}$

CAPÍTULO 3: SOLUÇÃO DAS EQUAÇÕES ANGULARES 20

3.1 - Auto-estados de Momento Angular- 21

3.2 - Soluções Exatas em Torno da Origem 23

3.3 - Resultados Analíticos para $R$ Finito 24

3.4 - Diagonalização pelas Funções de Laguerre-Jacobi 28

CAPÍTUlO 4: APLICAÇÕES 31

4.1 - Átomo de Hélio 32

4.2 - Aplicação da Diagonalização Laguerre-Jacobi para o Átomo de Hélio 56

4.3 - Excitons Ligados em Semicondutores

4.4 - Íon Negativo do Positrônio, $\mathrm{Ps}^{-} \quad 72$

$\begin{array}{ll}4.5-d d \mu \text { e } d_{2}^{+} & 75\end{array}$

CAPÍTULO 5: CONCLUSÕES $\quad 80$

APÊNDICES

A- Cálculo dos Coeficientes de Acoplamento Angular 83

B- Hyperspherical Formulation of Impurity-Bound Excitons

in Semiconductors $\quad 86$

C- General Theoretical Approach to Coulombic Three-Body

$\begin{array}{ll}\text { Systems by the Hyperspherical Formalism } & 95\end{array}$

$\begin{array}{ll}\text { BIBLIOGRAFIA } & 106\end{array}$ 


\section{Lista de Tabelas}

Tabela 1: Localização do pólos para vários sistemas físicos.

Tabela 2: Classificação das curvas de potencial para $\mathrm{L}=0 \operatorname{com} \ell_{1}=\ell_{2}=\ell$.

Tabela 3: Energias do estado fundamental do átomo de hélio

Tabela 4: Valores calculados de $U(R) / R^{2}$ (em i.u.) para a curva mais baixa do $I_{c}$ no seu ponto de mínimo, em função de $N$ e $\ell_{\max }$.

Tabela 5: Análise de convergência da diagonalização Laguerre-Jacobi no ponto de mínimo de $U_{0} / R^{2}$ para o $H_{e}$ atômico. Os índices $N_{\max }$ e $\ell_{\max }$ correspondem ao valor máximo de $N_{\lambda}$ e $\ell+1$ que definem os elementos da base. Os valores de comparação são fornecidos na tabela 4.

Tabela 6: Mesma análise de convergência da tabela 5, para a região assintótica com $R=10$.

Tabela 7: Valores do ponto de mínimo de $U(R) / R^{2}$ (em meV) para a curva mais baixa de um exciton ligado a uma impureza de carga $e$ no semicondutor $\mathrm{ZnSe}$.

Tạbela 8: Energia de ligação para excitons ligados a impurezas de carga e em vários materiais semicondutores. $E_{D}$ é a energia de dissociação. Os índices referem-se a cálculos variacionais: 1) referência 26 e 2) referência 27. As constantes dielétricas $\varepsilon$ são obtidas da referência 26.

Tabela 9: Energias ressonantes de excitons ligados a impurezas no $\mathrm{ZnSe}$ correspondentes a primeira curva excitada

Tabela 10: Análise de convergência da curva de potencial $-U(R)_{0} / R^{2}(\mathrm{em} \mathrm{eV})$ do $d d \mu$ para seu ponto de mínimo.

Tabela 11: Expressões para $t$ e $f$ da equação (69). 


\section{Lista de Figuras}

Figura 1: Pontos singulares da equação angular na variável $z$.

Figura 2: Potencial hiperesférico do átomo de hélio.

Figura 3: Densidade de probabilidade da solução angular 0.

Figura 4: Curvas de potencial mais baixas para o átomo de hélio.

Figura 5: Densidade de probabilidade da solução angular 1.

Figura 6: Densidade de probabilidade da solução angular 2.

Figura 7: Curva de potencial mais baixa do átomo de hélio com o estado fundamental e as primeiras energias excitadas.

Figura 8: Primeira curva de potencial excitada do átomo de hélio com as primeiras ressonâncias.

Figura 9: Segunda curva de potencial excitada do átomo de hélio com as primeiras ressonâncias.

Figura 10: Densidade de probabilidade radial correspondente às encrgias da figura 7 .

Figura 11: Densidade de probabilidade radial correspondente às energias da figura 8 .

Figura 12: Densidade de probabilidade radial correspondente às energias da figura 9 .

Figura 13: Densidade de probabilidade da função angular total correspondente a energia do estado fundamental.

Figura 14: Densidade de probabilidade da função angular total correspondente a segunda energia da curva 0.

Figura 15: Densidade de probabilidade da função angular total correspondente a terceira energia da curva 0.

Figura 16: Densidade de probabilidade da função angular total correspondente a primeira energia da curva 1. 
Figura 17: Densidade de probabilidade da função angular total correspondente a segunda energia da curva 1 .

Figura 18: Densidade de probabilidade da função angular total correspondente a primeira energia da curva 2 .

Figura 19: Densidade de probabilidade da funçào angular total correspondente a segunda energia da curva 2.

Figura 20: Potencial hiperesférico de um exciton ligado a uma impureza no semicondutor $\mathrm{ZnSe}$.

Figura 21: Curvas de potencial como função do número de canais angulares para o $\mathrm{ZnSe}$.

Figura 22: Curvas de potencial para diferentes materiais semicondutores em unidades adimensionais.

Figura 23: Curvas de potencial em meV para excitons ligados a impurezas.

Figura 24: Comportamento da energia de ligação de excitons ligados com relação a razão de massa $m$

Figura 25: Densidade de probabilidade radial de excitons ligados em função de $m$.

Figura 26: Comportamento do raio médio hiperesférico em função de $m$.

Figura 27: Curvas de potencial mais baixas para excitons ligados em impurezas no semicondutor $\mathrm{ZnSe}$.

Figura 28: Densidade de probabilidade radial de estados excitados de excitons ligados a impurezas no semicondutor $\mathrm{ZnSe}$.

Figura 29: Potencial hiperesférico para o $\mathrm{Ps}^{-}$.

Figura 30: Potencial hiperesférico para o sistema $d d \mu$.

Figura 31: Curvas de potencial em função do acoplamento de canais angulares para o sistema $d d \mu$.

Figura 32: Curva de potencial mais baixa do $d_{2}^{+}$com energias obtidas na aproximaçâo adiabática extrema. 


\section{RESUMO}

Nesse trabalho investigamos as propriedades de sistemas Coulombianos de três corpos usando coordenadas hiperesféricas. A partir da quasi-separabilidade da equação de Schrödinger nessas coordenadas desenvolvemos um procedimento que permite uma descrição unificada de sistemas atômicos, moleculares e ions exóticos. O método, denominado procedimento hiperesférico adiabático, permite o cálculo das propriedades do estado fundamental com grande precisão bem como, é adequado a análise de estados excitados, ressonâncias no contínuo c funções de espallıamento. Novas técnicas cle solução das equações diferenciais foram desenvolvidas de forma a incorporar efeitos de centro de massa a sistemas de massas díspares. Consequentemente foi possível a aplicação desta metodologia ao problema de excitons aprisionados em semicondutores, a mesomoléculas e a íons exóticos. Finalmente desenvolvemos uma nova técnica para o cálculo das curvas de potencial baseadas na construção de uma família de funções trancedentais ortogonais. O átomo de hélio é usado para o teste da eficiência do método. 


\begin{abstract}
Coulombic three-body systems are investigated using the hyperspherical adiabatic approach. By using a suitable variable $z=\operatorname{tg}(\alpha / 2)$ angular differential equation for the determination of the potential curves, we are able to obtain stable series expansion solutions, valid for small and large values of the hyperspherical radius. The analysis of the mathematical of the differential equations in the variable $z$, offers an insight into the physics of the problem and into the determination of stable converging solutions as well. In order to illustrate our investigation we apply this study to several carefully chosen systems: $H e, d d \mu, d_{2}^{+}$, and excitons boun Coulomb center in different semiconductors. Eigenenergies are obtained and compared with other methods. In this work we make use of a recently proved set o basic inequalities which provide, for the first time, a lower bound/upper bound for the ground state energies of the selected systems.
\end{abstract}

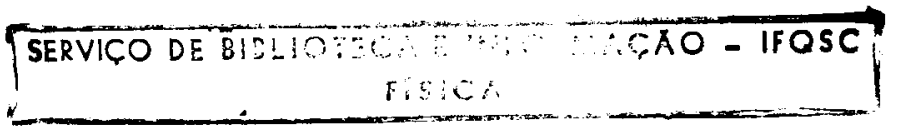


Capítulo 1

INTRODUÇÃO 
O problema Coulombiano de três corpos tem sido exaustivamente investigarlo teórica e experimentalmente descle os primórdios da mecünica quântica. Inicialmente os esforços foram direcionados ao estudo do estado fundamental de espécies típicas como o átomo de hélio e o íon molecular de hidrogênio, o $\mathrm{H}_{2}^{+}$. Na medida em que técnicas experimentais foram se desenvolvendo, tornando possivel a detegào de pecpuenas correçoes relativísticas, surgiu a necessidade de obtenção de funções de onda de alta precisão para estes sistemas. Nas últimas décadas novas possibilidades experimentais surgiram com o desenvolvimento de fontes de luz ultra violeta de vácuo. Propriedades como os estados autoionizantes do átomo de hélio puderam ser investigados [1]. Esse estados, estávcis na ausência da interação intereletrônica, decacm para o continuo quando as correlações cletrônicas são levadas em conta. Os átomos de dois elétrons como o $\mathrm{He}$ c o $\mathrm{H}^{-}$, bem como os íons moleculares $\mathrm{H}_{2}^{+}$e $D_{2}^{+}$se constituem nos exemplos mais simples não triviais, cujo estudo pode levar à compreenção de parte dos processos químicos que envolvam correlações eletrônicas. Do ponto de vista da física molecular ainda não lá uma comprecnsão satisfatória dos processos de dissociação molecular. O estudo destes fenômenos requer um formalismo que permita o cálculo do conjunto das propriedades de sistemas Coulombianos de três corpos de uma forma unificada e transparente.

Vários métodos para tratar do problema de três corpos são encontrados na literatura. O método variacional fornece energias precisas para o estado fundamental, no entanto sua eficiência é reduzida ao ser aplicado a estados excitados ou no contínuo. A mesma limitaçào é obscrvanda para o método das múltiplas configuraçós c para o de llartrec-Fock. 
Outra alternativa amplannente utilizarla em fisica atômica ć o mótodo das partículas independentes, onde a correlaçào entre as partículas mais leves é inicialmente desprezada. Este procedimento usa como ponto de partida combinações de soluções hidrogenóides, enquanto que os efeitos de correlação sào introduzidos perturbativamente, de forma controlada. En física molecular o movimento núclear é separado do eletronico num procedimento adiabático conhecido como método Born-Oppenheimer (BO). A equação eletrônica é resolvida para valores fixos das distâncias internucleares, gerando um conjunto infinito de curvas de potencial. Estes métodos, apesar da sua eficiência na análise de fenômenos específicos em física atômica e molecular, não permitem uma visão unificada dos sistemas de três corpos.

Coordenadas hiperesféricas são coordenadas esféricas em seis dimensões. Ao invés das variáveis $\left(\theta_{1}, \phi_{1}, r_{1}\right)$ e $\left(\theta_{2}, \phi_{2}, r_{2}\right)$ para cada uma das partículas, são introduzidas as variáveis $R=\sqrt{r_{1}^{2}+r_{2}^{2}} \mathrm{e} \alpha=t g^{-1} r_{1} / r_{2}$ que incorporam os aspectos coletivos do sistema. As coordenadas hiperesféricas correlacionam as variáveis radiais esféricas de tal forma que o movimento do sistema fica descrito por apenas uma coordenada radial, $R$, sendo as demais compactas. A equaçâo de Schrödinger em coordenadas hiperesféricas apresenta termos puramente radiais e termos angulares com uma dependência linear em $R$. A forma simples com que $R$ participa dos termos angulares sugere uma separação parcial da função de onda total do sistema em uma parte radial e outra angular, sendo que nesta, o hiper-raio participa de forma paramétrica. O procedimento descrito é conhecido como Método Hiperesférico Adiabático (HAA), e foi introduzido em física atômica em 
1968 por Macek [2]. Seu trabalho teve por objetivo o estudo das ressonâncias autoionizantes do átomo de hélio. Foram obtidos resultados para a posição dessas ressonâncias em concordância com dados experimentais. Listes estados podem ser representados qualitativamente por combinaçòes de funçòes hidrogenóides excitadas de dois elétrons, c decaem devido a sua correlação. No Método Iliperesférico Adiabático eles surgem de modo natural como autoestados de curvas de potencial, semellantes às de Born - Oppenheimer. Analogamente ao que ocorre no $\mathrm{BO}$, a variável $R$ é prefixada e a equação de Schrödinger resolvida como função paramétrica do hiper-raio gerando uma familia de curvas de potencial. Na medida em que os efeitos de correlaçào que caracterizam um sistema particular estão incluidos no cálculo destas curvas, elas contém as informações físicas fundamentais de cada uma das espécies. Assim, é de grande importância a precisão com que estas curvas são calculadas.

O procedimento tradicional para obtenção das curvas de potencial consiste na expansão da função de onda angular em suas soluções exatas para $R=0$, os hiperesféricos harmônicos. A diagonalizaçào do hamiltoniano com esta base apresenta bons resultados para potenciais de curto alcance, como o potencial nuclear[3,4]. No caso atômicomolecular a convergência da expansão em hiperesféricos harmônicos se torna cada vêz mais lenta para valores crescentes de $R$ devido ao longo alcance das forças Coulombianas. Neste limite o sistema está perto do limiar de ionização, e a função de onda é do tipo hidrogenóide, ou scja, funçòs de Laguerre. $\Lambda$ literatura contém uma enorme diversidade de contribuições para o problema, desenvolvidas basicamente pelo grupo de U. Fano [5- 
9]. Inicialmente os esforços se concentraram na obtenção semi-quantitativa de curvas de potencial, na classificação de estados e na generalização dos métodos a outros sistemas. Estes esforços, não foram suficientes para a transformação do HAA num método quantitativamente competitivo na medida em que as obstruções a acuidade não foram eliminadas. As dificuldades citadas foram resolviclas parcialunconte em 1986 por Ilornos, MacDowell e C.D.Caldwell [10] com a introdução da variável $z=\operatorname{tg}(\alpha / 2)$, que transforma os coeficientes da equação angular em funções racionais. Na sua forma original estes coeficientes contém funções trigonométricas, de dificil expansão. Com isso, o sistema de equações angular pode ser resolvido diretamente através da expansão das funções angulares, tambem chamadas de funções de canal, em séries de potências. Incorporando à solução propriedades analíticas de $R=0$ e $R \rightarrow \infty$, obteve-se pela primeira vêz técnicas eficientes para o cálculo de curvas de potencial e, consequentemente, resultados não adiabáticos para o estado fundamental do átomo de hélio acoplando vários estados de momento angular e resolvendo-se duas equações radiais acopladas. Considerando no método HAA uma única curva de potencial e calculanclo os níveis de energia suportados por esta curva, obtem-se valores aproximados para as energias de ligação do sistema. Essa aproximação drástica é denominada aproximação adiabática extrema (EAA). O passo seguinte consiste em permitir a interação entre essas curvas dando origem a uma sucessão de aproximações denominadas aproximação adiabática acoplada (CAA). Em 1991 [11] provou-se que a energia do estado fundamental do problema de três corpos obedece a desigualdade $\varepsilon_{E A A} \leq \varepsilon \leq \varepsilon_{C A A}$. Ficam assim estabelecidos limites inferiores e superiores para a enegia 
exata $\varepsilon$.

Nesta tese generaliza-se o método de expansão das funções angulares na variável $z$ de modo que possa ser aplicado ao problema geral de três corpos. Inicialmente as técnicas desenvolvidas sio introdugidas em física da maléria condensada no cestudo de excilons lig-

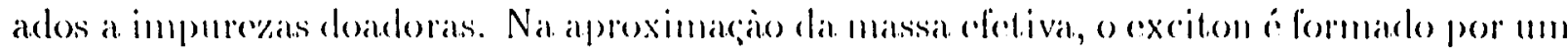
par elétron - buraco com massas c cargas efetivas que simulam o efeito da rede de material semicondutor . A existência de massas distintas, que caracteriza os excitons aprisionados, requer a redefiniçào das condiçoes de contorno apropriadas, e a consequente solução das equações nessas novas condições de contorno. A repulsão do buraco pela impureza, considerada fixa, faz com que a energia de ligação do sistema seja muito pequena, tornando critico o acoplamento de muitos estados de momento angular. Os resultados obtidos para energias de ligação, funções de onda para vários materiais na aproximação adiabática extrema, e uma análise do problema da massa crítica estão detalhados na referência [12], apêndice A. A seguir, os esforcos foram direcionados para os sistemas moleculares, onde os efeitos de centro de massa devem ser incorporados à solução. Devido a estrutura de singularidades do sistema cle equaçòes, a expansào em séries de potencias da função de onda deste problema é complexa, exigindo, técnicas especiais que evitem a propagação de erros. A solução encontrada consiste cm transladar a equação angular de um intervalo $z_{0}$, definindo $z \rightarrow z^{\prime}+z_{0}$. Esta transformação nào muda a forma polinômial dos coeficientes do conjunto de çuaçòes diferenciais, permitindo a propagaçào das funçòes de canal em intervalos estabelecidos pela precisão desejada. Outra aplicação foi feita com o estudo do 
ín negativo do pósitron, o $\mathrm{Ps}^{-}$. Seu interesse é devido à possibilidade de aniquilamento elétron-pósitron, mas também, por ser constituido de partículas de massa comparável.

Visando demonstrar a competitividade do método hiperesférico no cálculo preciso de energias de ligação, curvas de potencial com dez canais de momento angular acoplados foram obtidas e termos de acoplannento para três canais radiais foram calculados para o átomo de hélio [13].

Finalmente, uma técnica de obtenção de uma base para diagonalização do operador angular, que chamamos de Diagonalizaçào Laguerre-Jacobi [14], é apresentada. A principal diferença com relaçào aos métodos de diagonalização tradicionais é a da inclusão de informações do potencial hiperesférico nos elementos da base, o que conduz a resultados convergentes tanto para $R=0$ quanto para $R \rightarrow \infty$. Estes elementos são gerados a partir dos termos desacoplados da equaçào angular. Com isso, no limite $R=0$ a base é formada por polinômios de Jacobi, e para $R \rightarrow \infty$ polinômios de Laguerre. O desenvolvimento de um método do diagonalizaçào eficionte tem por objetivo uma aplicação futura em problemas de quatro corpos, como o Lítio, onde a solução direta das equações acopladas é dificultada pela complexidade destes sistemas. Como teste procurou-se reproduzir os valores de curva de potencial do átomo de hélio, obtidos pelo acoplamento das equações angulares. Os bons resultados indicam que este método pode vir a se tornar um importante meio de tratar problemas mais complexos.

A estrutura deste trabalho é fornecida a seguir. No capítulo II desenvolve-se o método hiperesférico adiabático, onde é descrita a separaçào das partes radiais e angular da função 
de onda total do sistema. A forma com que sào obtidas as funções de canal e curvas de potencial, está descrita no capítulo III. No capítulo IV o procedimento matemático desenvolvido neste trabalho é aplicado a vários sistemas de interesse. O capítulo V é dedicado as conclusões. No apêndice $\Lambda$ estão os coeficientes de acoplamento angular, e nos apêndices $\mathrm{B}$ a C os trabalhos já publicados, onde parte do material dessa tese é detalhado. 
Capítulo 2

O Método Hiperesférico

Adiabático 


\subsection{Formalismo Hipersférico}

No referêncial de laboratório define-se $\vec{r}_{i}$ como a coordenada da partícula $i$ com massa $m_{i}$. Para o problema de três corpos o conjunto das coordenadas de Jacobi é apropriado por gerar um hamiltoniano onde os termos referentes ao movimento do centro de massa $\vec{\rho}_{0}$ (c.m.) podem ser separados. Seja o vetor $\vec{\rho}_{1}$ da partícula 1 para a partícula 2 com massa reduzida, $\mu_{1}=m_{1} m_{2} /\left(m_{1}+m_{2}\right)$. O segundo vetor $\vec{\rho}_{2}$, liga o centro de massa das partículas 1 e 2 à partícula 3, com massa reduzida $\mu_{2}=\left(m_{1}+m_{2}\right) m_{3} /\left(m_{1}+m_{2}+m_{3}\right)$.

Para simplificar a forma do hamiltoniano, é util definir as coordenadas, $\vec{\xi}_{i}=\left(\mu_{i} / \mu\right)^{1 / 2} \vec{\rho}_{i}$, onde $\mu$ é uma massa arbitrária. A forma das coordenadas por extenso é,

$$
\begin{aligned}
& \vec{\xi}_{0}=\left(m_{1} \vec{r}_{1}+m_{2} \vec{r}_{2}+m_{3} \vec{r}_{3}\right) /\left(m_{1}+m_{2}+m_{3}\right), \\
& \vec{\xi}_{1}=\left(\mu_{1} / \mu\right)^{1 / 2}\left(\vec{r}_{1}-\vec{r}_{2}\right), \\
& \vec{\xi}_{2}=\left(\mu_{2} / \mu\right)^{1 / 2}\left(\frac{m_{1} \vec{r}_{1}+m_{2} \vec{r}_{2}}{m_{1}+m_{2}}-\vec{r}_{3}\right) .
\end{aligned}
$$

Assim, o hamiltoniano do sistema de três corpos interagindo via potencial de Coulomb é escrito como,

$$
H=-\frac{h^{2}}{2\left(m_{1}+m_{2}+m_{3}\right)} \nabla_{\vec{\xi}_{0}}^{2}-\frac{h^{2}}{2 \mu}\left(\nabla_{\vec{\xi}_{1}}^{2}+\nabla_{\vec{\xi}_{2}}^{2}\right)+V\left(\vec{\xi}_{1}, \vec{\xi}_{2}\right),
$$

onde

$$
V\left(\vec{\xi}_{1}, \vec{\xi}_{2}\right)=\sqrt{\frac{\mu_{1}}{\mu}} \frac{q_{1} q_{2}}{\xi_{1}}+\frac{m_{1}}{\sqrt{1 \mu_{1}}} \frac{q_{1} q_{3}}{\left|\vec{\xi}_{1}+\eta_{+} \vec{\xi}_{2}\right|}+\frac{m_{2}}{\sqrt{1 \mu \mu_{1}}} \frac{q_{2} q_{3}}{\left|\vec{\xi}_{1}-\eta_{-} \vec{\xi}_{2}\right|}
$$

é o potencial e,

$$
\eta_{+}=\left[m_{1}\left(m_{1}+m_{2}+m_{3}\right) / m_{2} m_{3}\right]^{1 / 2}
$$




$$
\eta_{-}=\left[m_{2}\left(m_{1}+m_{2}+m_{3}\right) / m_{1} m_{3}\right]^{1 / 2}
$$

Devido a esta escolha de coordenadas o movimento do centro de massa é separado em seu operador de energia cinética. Removendo este termo passa-se a utilizar as coordenadas hiperesféricas. O raio hiperesférico é definido como

$$
R^{2}=\xi_{1}^{2}+\xi_{2}^{2}
$$

e o angulo hiperesférico $\alpha$,

$$
\begin{aligned}
\xi_{1} & =R \operatorname{sen} \alpha \\
\xi_{2} & =R \cos \alpha \\
\operatorname{tg}(\alpha) & =\frac{\xi_{1}}{\xi_{2}} \quad 0 \leq \alpha \leq \pi / 2 .
\end{aligned}
$$

Com esta mudança, passa-se do conjunto de duas coordenadas radiais e quatro compactas $\left(\xi_{1}, \xi_{2}, 0_{1}, \theta_{2}, \phi_{1}, \phi_{2}\right)$ para um conjunto com apenas uma coordenada radial e cinco compactas $\left(R, \alpha, \theta_{1}, \theta_{2}, \phi_{1}, \phi_{2}\right)$. Utilizando as coordenadas hiperesféricas a equação de Schrödinger $I V=$ E

$$
\left[\frac{\partial^{2}}{\partial R^{2}}-\frac{U(R, \Omega)-1 / 4}{R^{2}}+\varepsilon\right] \psi(R, \Omega)=0,
$$

$\operatorname{com} \varepsilon=\left(2 \mu / \hbar^{2}\right) E$, onde $E$ é o autovalor energia e $\Omega=\left\{\alpha, \hat{\xi}_{1}=\left(0_{1}, \varphi_{1}\right), \hat{\xi}_{2}=\left(0_{2}, \varphi_{2}\right)\right\}$. O termo $\psi(R, \Omega)$ corresponde à funçào de onda renormalizada dada por $\psi(R, \Omega)=$ $R^{5 / 2} \operatorname{sen} \alpha \cos \alpha \Psi(R, \Omega)$. Esta escolha gera um hamiltoniano onde as derivas hiperesféricas radial e angular não são cruzadas. O operador angular $U(R, \Omega)$ contém os operadores das variáveis compactas, c o hiper-raio $R$ numa forma linear segundo a relação,

$$
U(R, \Omega)=U(0)+R^{2} v(R, \Omega)=U(0)+R \hat{v}(\alpha, \theta)
$$


onde o termo de cnergia cinctica

$$
I(0)=-\frac{\partial^{2}}{\partial \alpha^{2}}+\frac{\hat{\ell}_{1}^{2}\left(\hat{\xi}_{1}\right)}{\operatorname{sen}^{2} \alpha}+\frac{\hat{\ell}_{2}^{2}\left(\hat{\xi}_{2}\right)}{\cos ^{2} \alpha}
$$

é função dos opcradores de monconto angular individuais $\hat{\ell}_{i}^{2}\left(\hat{\xi}_{i}\right)(i=1,2)$, relativos às

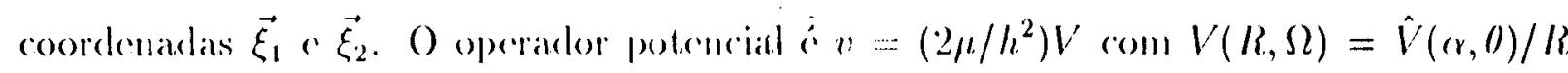
onde,

$$
\begin{aligned}
\hat{V}(\alpha, \theta) & =\sqrt{\frac{\mu_{1}}{\mu}} \frac{q_{1} q_{2}}{\operatorname{sen} \alpha}+\frac{m_{1}}{\sqrt{\mu \mu_{1}}} q_{1} q_{3} / \sqrt{\operatorname{sen}^{2} \alpha+\eta_{+}^{2} \cos ^{2} \alpha+\eta_{+} \operatorname{sen} 2 \alpha \cos \theta} \\
& +\frac{m_{2}}{\sqrt{\mu \mu_{1}}} q_{2} q_{3} / \sqrt{\operatorname{sen}^{2} \alpha+\eta_{-}^{2} \cos ^{2} \alpha-\eta_{-} \operatorname{sen} 2 \alpha \cos \theta}
\end{aligned}
$$

Em coordenadas esfericas o ângulo entre os versores $\hat{\xi}_{1}$ e $\hat{\xi}_{2}$ ć definido como,

$$
\cos \theta=\hat{\xi}_{1} \cdot \hat{\xi}_{2}=\cos \left(\theta_{1}\right) \cos \left(\theta_{2}\right)+\operatorname{sen}\left(\theta_{1}\right) \operatorname{sen}\left(\theta_{2}\right) \cos \left(\phi_{1}-\phi_{2}\right)
$$

\subsection{Procedimento Hiperesférico Adiabático}

A equaçào de autovalores (7) 6 formada por uma parte puramente radial e outra. angular, cuja dependência na variável $R$ é lincar, sugerindo uma separação da função de onda. Assim, no chamado Procedimento Adiabático Hiperesférico (HAA), R será considerado como parâmetro adiabático para a expanção da função de onda total do sistema na forma, 


$$
\psi(R, \Omega)=\sum_{\lambda} F_{\lambda}(R) \Phi_{\lambda}(R ; \Omega)
$$

A base $\Phi_{\lambda}$ é conhecida como funçào de canal é obtida da equação de autovalores,

$$
U(R, \Omega) \Phi_{\lambda}(R ; \Omega)=U_{\lambda}(R) \Phi_{\lambda}(R ; \Omega)
$$

que é resolvida para valores fixos de R. O conjunto de números quânticos $\lambda$, que classificam as funções de canal, serão discutidos no próximo capítulo. Substituindo a expansão (22) em (7) e utilizando a ortonormalidade de $\Phi_{\lambda}$, obtem-se a equação de autovalores para a função de onda radial $F_{\lambda}(R)$,

$$
\left[\frac{d^{2}}{d R^{2}}-\frac{U_{\lambda}(R)-1 / 4}{R^{2}}+\varepsilon_{\lambda}\right] F_{\lambda}(R)+\sum_{\lambda^{\prime}} W_{\lambda \lambda^{\prime}}(R) F_{\lambda^{\prime}}(R)=0 .
$$

Os termos não adiabáticos, que causam o acoplamento através dos elementos da base angular são dados por,

$$
W_{\lambda \lambda^{\prime}}=2 P_{\lambda \lambda^{\prime}}(R) \frac{d}{d R}+Q_{\lambda \lambda^{\prime}}(R)
$$

onde,

$$
P_{\lambda \lambda^{\prime}}(R)=<\Phi_{\lambda}\left|\frac{d}{d R}\right| \Phi_{\lambda^{\prime}}>
$$

e,

$$
Q_{\lambda \lambda^{\prime}}(R)=<\Phi_{\lambda}\left|\frac{d^{2}}{d R^{2}}\right| \Phi_{\lambda^{\prime}}>
$$

Os elementos $P_{\lambda \lambda^{\prime}}$ c $Q_{\lambda \lambda^{\prime}}$ sào relacionados diferenciando $P_{\lambda \lambda^{\prime}}(R)$ com relação a $R$ :

$$
\frac{d P_{\lambda \lambda^{\prime}}}{d R}=Q_{\lambda \lambda^{\prime}}+R_{\lambda \lambda^{\prime}}
$$


onde,

$$
R_{\lambda \lambda^{\prime}}(R)=<\frac{d \Phi_{\lambda}}{d R} \mid \frac{d \Phi_{\lambda^{\prime}}}{d R}>
$$

Assumindo a completera da basce,

$$
R_{\lambda \lambda^{\prime}}=\sum_{\tau} P_{\lambda \tau} P_{\tau \lambda^{\prime}}
$$

Para ser resolvido numericamente, o conjunto infinito de equaçòes (14) deve ser truncado, resultando na Aproximação Adiabática Acoplada (CAA). O desacoplamento das equaçoes radiais negligenciando os elementos $W_{\lambda \lambda^{\prime}}(R)$ para $\lambda \neq \lambda^{\prime}$, corresponde à Aproximaçào Adiabática Desacoplada (UA $\Lambda)$. No caso de $W_{\lambda \lambda}(R)$ também ser negligenciado, define-se a Aproximação Adiabática Extrema (EAA). Foi demonstrado [11] que para as energias do estado fundamental é válicla a incgualdade $\varepsilon_{E A A} \leq \varepsilon \leq \varepsilon_{C A A} \leq \varepsilon_{U A A}$, onde $\varepsilon$ é a energia exata. O EAA fornece um limite inferior a energia, enquanto que os UAA e CAA levam a limites superiores, como no procedimento variacional. Com o cálculo preciso das funções de canal angular, as correções podem ser acrescentadas gradualmente, até o resultado convergir para o valor de encrgia exato.

$\mathrm{O}$ sistema de $\mathrm{N}$ equações acopladas resultante do truncamento, deve gerar soluções e derivadas contínuas em $R=0$ e $R=\infty$. Para fazer a integração numérica, a função de onda será propagada analiticamente a partir de $R=0$ através da expansão,

$$
F_{\lambda}(R)=R^{S_{\grave{\lambda}}}(\ln R)^{q} \sum_{I=0}^{\infty} \sum_{K=0}^{\infty} f_{\lambda}^{\dot{\lambda}}(I, K) R^{I}(\ln R)^{K}
$$


Neste limite $P_{\lambda \lambda^{\prime}}$ e $Q_{\lambda \lambda^{\prime}}$ são regulares, cunquanto que $U_{\lambda}$ assume o valor intciro $m_{\lambda}^{2}$ (Sec. III.2) . Desta forma a W(. (14) possui un polo $\left(\left(-m_{\lambda}^{2}+1 / 1\right) / R^{2}\right)$ que é climinado escollendo $S_{\lambda}=\left(m_{\lambda}+1 / 2\right)$. As singularidades logaritmicas surgem quando $\left(m_{\lambda}-m_{\dot{\lambda}}\right)$ são números pares positivos. A existência destas singularidades na variável $R$ foi estabelecida por Bartlett [15]. A forma da expansào (21) foi proposta por Fock [16] para ser utilizada em cálculos variacionais do estado fundamental do átomo de hélio $[17,18]$, sendo recentemente[10] introduzida no método hiperesférico adialbático.

Os termos não adiabáticos e as curvas de potencial tambem são expandidos, gerando

$$
P_{\lambda \lambda^{\prime}}(R)=\sum_{n=0}^{\infty} P_{\lambda \lambda^{\prime}}^{(n)} R^{n} \quad Q_{\lambda \lambda^{\prime}}(R)=\sum_{n=0}^{\infty} Q_{\lambda \lambda^{\prime}}^{(n)} R^{n}
$$

e,

$$
U_{\lambda}(R)=m_{\lambda}^{2}+\sum_{n=1}^{\infty} U_{\lambda}^{(n)} R^{n} .
$$

Para o limite $R \rightarrow \infty$ utiliza-se a expansão assitótica,

$$
F_{\lambda}(R)=R^{S_{\lambda}} e^{-K_{\lambda} R} \sum_{n=0}^{\infty} \frac{G_{\lambda}^{(n)}}{R^{n}}
$$

onde

$$
K_{\hat{\Lambda}}^{2}=-2 E-\operatorname{Lim}_{N-\infty} U_{\lambda}(R)
$$

Ao contrário de $R=0$, a expansào das curvas de potencial depende do conjunto de coordenadas de Jacobi escolhido [19]. Na forma geral definc-se,

$$
\frac{U_{\lambda}}{R^{2}}=\sum_{n=0}^{\infty} \frac{U_{\lambda}^{(n)}}{R^{n}}
$$


A cxpansão dos termos nào adiabáticos é,

$$
\frac{P_{\lambda \Lambda^{\prime}}}{R^{2}}=\sum_{n=1}^{\infty} \frac{P_{\lambda \Lambda^{\prime}}^{(n)}}{R^{n}} \quad \frac{Q_{\lambda \Lambda^{\prime}}}{R^{2}}=\sum_{n=2}^{\infty} \frac{Q_{\lambda \Lambda^{\prime}}^{(n)}}{R^{n}}
$$

As condições iniciais de propagação devem ser obtidas com grande precisão para evitar erros numéricos. Com este objetivo apresentia-se na próxima seção expressões analíticas que podem ser utilizadas em conjunto com processos de diagonalização $[13,20]$ para gerar os elementos das expansões descritas acima.

\subsection{Propriedades da Equação Angular}

A equaçào angular (13) pode ser explorada de modo a fornecer métodos que permitam um cálculo numérico eficiente dos termos de acoplamento nào adiabáticos (Eq. 16 e 17) e das derivadas das curvas de potencial. A inclinação da curva ć obticla seguindo o seguinte procedimento: inicialmente calcula-se a derivada da expressão (13) com relação a $R$,

$$
\hat{v}(\alpha, \theta) \Phi_{\lambda}(R ; \Omega)+\left[U(R, \Omega)-U_{\lambda}(R)\right] \frac{d}{d R} \Phi_{\lambda}(R ; \Omega)=\frac{d}{d R} U_{\lambda}(R) \Phi_{\lambda}(R ; \Omega) .
$$

Multiplicando a Eq.(2S) por $\Phi_{\lambda^{\prime}}(R ; \Omega)$ e integrando sobre $d \Omega$, obtem-se

$$
\begin{aligned}
\left\langle\Phi_{\lambda^{\prime}}(R ; \Omega)|\hat{v}(\alpha, 0)| \Phi_{\lambda}(R ; \Omega)\right\rangle+\left\langle\Phi_{\lambda^{\prime}}(R ; \Omega)\right| U(R, \Omega)- & U_{\lambda}(R)\left|\Phi_{\lambda}(R ; \Omega)\right\rangle \\
& =\frac{d}{d R} U_{\lambda}(R) \delta_{\lambda^{\prime} \lambda^{\prime}} .
\end{aligned}
$$

Assim, para $\lambda^{\prime}=\lambda$

$$
\frac{d}{d R} U_{\lambda}(R)=\left\langle\Phi_{\lambda}|\hat{v}(\alpha, 0)| \Phi_{\lambda}\right\rangle
$$

e, para $\lambda^{\prime} \neq \lambda$,

$$
\left\langle\Phi_{\lambda^{\prime}}|\hat{v}(\alpha, \theta)| \Phi_{\lambda}\right\rangle=-\left(U_{\lambda^{\prime}}-U_{\lambda}\right) P_{\lambda^{\prime} \lambda}(R),
$$


ou, de modo equivalente,

$$
P_{\lambda^{\prime} \lambda^{\prime}}(R)=-\frac{\left\langle\Phi_{\lambda^{\prime}}|\hat{v}(\alpha, \theta)| \Phi_{\lambda}\right\rangle}{U_{\lambda^{\prime}}-U_{\lambda}}
$$

Esta forma de obter o $P_{\lambda^{\prime},}$ evita o cálculo da derivada das funçóes de canal, garantindo uma grande precisào do cálculo numérico. A repetiçào do mesmo procedimento para a derivada segunda da Eq. (13) resulta nas expressões abaixo.

$\operatorname{Para} \lambda^{\prime}=\lambda$;

$$
\frac{d^{2}}{d R^{2}} U_{\lambda}(R)=2\left\langle\phi_{\lambda}|\hat{v}| \frac{d}{d R} \phi_{\lambda}\right\rangle=2 \sum_{\tau \neq \lambda} P_{\lambda \tau}^{2}\left(U_{\lambda}-U_{\tau}\right)
$$

$\operatorname{para} \lambda^{\prime} \neq \lambda$

$$
Q_{\lambda \lambda^{\prime}}=\frac{\left[2 \frac{d}{d R} U_{\lambda^{\prime}} P_{\lambda \lambda^{\prime}}-2\left\langle\phi_{\lambda}|\hat{v}| \frac{d}{d R} \phi_{\lambda^{\prime}}\right\rangle\right]}{\left(U_{\lambda}-U_{\lambda^{\prime}}\right)}
$$

onde,

$$
\left\langle\phi_{\lambda}|\hat{v}| \frac{d}{d R} \phi_{\lambda^{\prime}}\right\rangle=\frac{d}{d R} U_{\lambda} P_{\lambda \lambda^{\prime}}+\sum_{\tau \neq \lambda} P_{\lambda \tau} P_{\tau \lambda}\left(U_{\tau}-U_{\lambda}\right)
$$

As propriedades obtidas nesta seção se aplicam a qualquer valor de $R$, mas são de grande importância para $R=0$. Neste limite todas as funções presentes na equação radial devem ser expandidas em sćries de potencias de $R$ para que a funçào de onda possa ser obtida. Pequenos erros no início de sua propagaçào numérica podem acabar por comprometer os resultados. 
Capítulo 3

Solução das Equações Angulares 


\subsection{Auto-Estados de Momento Angular}

A equação angular tem que ser desenvolvida de modo a permitir a construção de algoritmos numéricos eficientes. Valores como $U_{\lambda}(R)$ devem ser obtidos com precisão para todo domínio de $R$ e quaisquer valores de massas das partículas, permitindo o estudo tanto sistemas atômicos quanto moleculares.

O operador angular apresenta pólos em $\alpha=0$ e $\pi / 2$, referentes ao termo de energia cinética na Eq.(9), que não dependem das massas do sistema. No caso do termo de potencial, expresso pela Eq.(10), os pólos estào localizados em $\alpha=0,(0, \alpha)=\left(\pi, \alpha_{+}\right)$ e $(\theta, \alpha)=\left(0, \alpha_{-}\right)$, onde $\lg \alpha_{ \pm}=\eta_{ \pm}$. As funçòes angulares serão obtidas considerando soluções regulares nas três regiōes, $0 \leq \alpha \leq \alpha_{-}, \alpha_{-} \leq \alpha \leq \alpha_{+}$e $\alpha_{+} \leq \alpha \leq \pi / 2$, impondo condições de continuidade nos pontos de ligaçào.

Para eliminar os termos quadráticos $\sec (\alpha)$ e $\operatorname{cosec}(\alpha)$ que divergem fortemente, as funções de canal serâo expandidas da seguinte lorma,

$$
\Phi_{\lambda}(R ; \Omega)=\sum_{\ell_{1} \ell_{2}}(\operatorname{sen} \alpha)^{\ell_{1}+1}(\cos \alpha)^{\ell_{2}+1} \mathcal{Y}_{\ell_{1} \ell_{2}}^{L M}\left(\hat{\xi}_{1}, \hat{\xi}_{2}\right) \mathcal{G}_{\ell_{1} \ell_{2}}^{\lambda}(R ; \alpha)
$$

onde $\mathcal{Y}_{\ell_{1} \ell_{2}}^{L M}$ é a funçào de momento angular acoplado de cluas partículas definidas pelas coordenadas de Jacobi, com, $\left(\left|\ell_{1}-\ell_{2}\right| \leq l \leq \ell_{1}+\ell_{2}\right.$, e $\left.M=m_{1}+m_{2}\right)$. A escolha desta base tem a notivaçào de preservar a individualidade das partículas com relaçào ao movimento angular no campo de $\left(\theta_{1}, \varphi_{1}\right),\left(\theta_{2}, \varphi_{2}\right)$.

A função $\mathcal{G}_{\ell_{1} \ell_{2}}^{\lambda}(R ; \alpha)$ será calculada através da equaçào de autovalores, sem ser ex- 
pandida em qualquer base. A partir daqui, o tratamento utilizado neste trabalho difere das técnicas usuais que sào bascadas en algum tipo de expansão com polinômios ortogonais, ou outro tipo de funções. Estes métodos de diagonalização truncada assumem a separabilidade das variáveis $R$ e $\alpha$, ou seja,

$$
\Phi_{\lambda}(R ; \Omega)=\sum_{n \ell_{1} \ell_{2}} \chi_{n \ell_{1} \ell_{2}}^{\lambda}(R) \mathcal{Y}_{n \ell_{1} \ell_{2}}^{L M}(\Omega)
$$

onde o conjunto $\mathcal{Y}_{n \ell_{1} \ell_{2}}^{L M}$ são os hiperesféricos harmônicos. Esta escolha corresponde a fazer,

$$
\mathcal{G}_{\ell_{1} \ell_{2}}^{\lambda}(R ; \alpha)=\sum_{n} \chi_{n \ell_{1} \ell_{2}}^{\lambda}(R) P_{n}^{\ell_{1}+1 / 2, \ell_{2}+1 / 2}(\cos 2 \alpha) .
$$

O termo $P_{n}^{\ell_{1}+1 / 2, \ell_{2}+1 / 2}$ é o polinômio de Jacobi, o que gera problemas de conver gência para grandes valores de R, pois nesta região a função de onda é dominada por funções de Laguerre.

O procedimento adotado neste traballio corresponde a resoluçào direta do conjunto infinito de equaçôes diferenciais acopladas, que săo obtidas substituindo a expressão (36) na equação (13), resultando

$$
\begin{aligned}
{\left[\frac{d^{2}}{d \alpha^{2}}\right.} & +2\left[\left(\ell_{1}+1\right) \operatorname{cotg} \alpha-\left(\ell_{2}+1\right) \operatorname{tg} \alpha\right] \frac{d}{d \alpha}-U_{\lambda}(R) \\
& \left.-\left(\ell_{1}+\ell_{2}+2\right)^{2}\right] \mathcal{G}_{\ell_{1} \ell_{2}}^{\lambda}(R ; \alpha)-R \sum_{\ell_{1}^{\prime} \ell_{2}^{\prime}} \tilde{v}_{\ell_{1} \ell_{2} \ell_{1}^{\prime} \ell_{2}^{\prime}}^{L M}(\alpha) \mathcal{G}_{\ell_{1}^{\prime} \ell_{2}^{\prime}}^{\prime}(R ; \alpha)=0,
\end{aligned}
$$

onde,

$$
\check{v}_{\ell_{1} \ell_{2} \ell_{1}^{\prime} \ell_{2}^{\prime}}^{L M}(\alpha)=(\operatorname{sen} \alpha)^{\ell_{1}^{\prime}-\ell_{1}}(\cos \alpha)^{\ell_{2}^{\prime}-\ell_{2}}<\mathcal{Y}_{\ell_{1} \ell_{2}}^{L M}|\hat{v}| \mathcal{Y}_{\ell_{1}^{\prime} \ell_{2}^{\prime}}^{L M}>
$$


A forma explicita de $\langle\hat{v}\rangle$ é fornecida no Apêndice A.

\subsection{Soluções Exatas em Torno da Origem}

No limite $R=0$ as interações nào participam da equação angular, que passa a ser desacoplada. Quando isto ocorre, a le. (13) admite soluçoes exatas que sào independentes do tipo de sistema de três corpos. Para obte-las faz-se a mudança de variável, $\alpha=\alpha^{\prime} / 2$ e a transformação,

$$
\tilde{\mathcal{G}}_{\ell_{1} \ell_{2}}^{\lambda}(R ; \alpha)=(\operatorname{sen} \alpha)^{\ell_{1}+1}(\cos \alpha)^{\ell_{2}+1} \mathcal{G}_{\ell_{1} \ell_{2}}^{\lambda}(R ; \alpha)
$$

obtendo,

$$
\left[-\frac{d^{2}}{d \alpha^{\prime 2}}+\frac{4 \ell_{1}\left(\ell_{1}+1\right)}{16 s \epsilon n^{2}\left(\alpha^{\prime} / 2\right)}+\frac{4 \ell_{2}\left(\ell_{2}+1\right)}{16 \cos ^{2}\left(\alpha^{\prime} / 2\right)}+\frac{1}{4} U_{\lambda}(0)\right] \tilde{\mathcal{G}}_{\ell_{1} \ell_{2}}^{\prime}(0, \alpha)=0
$$

Esta equação pode ser comparada com a forma canônica da equação de Jacobi [21],

$$
\left[-\frac{d^{2}}{d \alpha^{\prime 2}}-\frac{1-4 a^{2}}{16 \operatorname{sen}^{2}\left(\alpha^{\prime} / 2\right)}-\frac{1-4 b^{2}}{16 \cos ^{2}\left(\alpha^{\prime} / 2\right)}-\left(\lambda+\frac{a+b+1}{2}\right)^{2}\right] \mathcal{Q}_{\lambda}^{(a, b)}\left(\cos \alpha^{\prime}\right)=0,
$$

que tem a soluçào regular,

$$
\mathcal{Q}_{\lambda}^{(a, b)}\left(\cos \alpha^{\prime}\right)=\left(\operatorname{sen}\left(\alpha^{\prime} / 2\right)\right)^{a+1 / 2}\left(\cos \left(\alpha^{\prime} / 2\right)\right)^{b+1 / 2} P_{\lambda}^{(a, b)}\left(\cos \alpha^{\prime}\right)
$$

onde $P_{\lambda}^{(a, b)}$ são os polinômios de Jacobi. Os autovalores, que correspondem aos valores das curvas de potencial para $R=0$, são da forma,

$$
U_{\lambda}(0)=m_{\lambda}^{2}=\left(2 n+\ell_{1}+\ell_{2}+2\right)^{2}
$$

e as funções $\tilde{\mathcal{G}}$ são dadas por,

$$
\tilde{\mathcal{G}}_{n \ell_{1} \ell_{2}}^{L M}(0, \alpha)=(\operatorname{sen} \alpha)^{\ell_{1}+1}(\cos \alpha)^{\ell_{2}+1} P_{n}^{\ell_{1}+1 / 2, \ell_{2}+1 / 2}(\cos (2 \alpha)) .
$$


A função de onda espacial fica definida pelos números quânticos $L, M$, spin $S$ e paridade. Em $R=0$ verifica-se que as curvas de potencial são classificadas pelo conjunto $\lambda=$ $\left(n, \ell_{1}, \ell_{2}\right)$. Para simplificar a notaçào serão omitidos $L, M, S$ e paridade. Os valores de $\ell_{1}$ e $\ell_{2}$ dependem da relação triangular com $L$, e a simetria do problema será imposta pela paridade. Nào há restriçòes quanto aos valores intciros de $n$.

\subsection{Soluções Analíticas para $R$ Finito}

A dificuldade na obtenção de soluçõcs para a Eq.(39), deve-se às formas trigonométricas presentes em seus coeficientes. Este fato impede o uso do teorema de Cauchy, uma vez que a expansão destes termos é numericamente intratável . Uma expansão de seus termos na variável $\alpha$ apresenta bons resultados apenas para grandes valores de $\mathrm{R}$, e mesmo assim, apenas para poucas cquagòes acopladas. ('om a intensào de transformar esta equação em outra, cujos coeficientes sejam funçòes racionais, descobriu-se a nova variável $z=\operatorname{g}(\alpha / 2)$. Assim, torna-se possivel obler soluçoes analiticas atraves de expansão em série de potencias da funçio de onda. (Com a variaivel z, a lep.(39) passa a forma,

$$
\begin{aligned}
{\left[\frac{d^{2}}{d z^{2}}\right.} & \left.+\mathcal{P}_{\ell_{1} \ell_{2}}(z) \frac{d}{d z}+\mathcal{Q}_{\ell_{1} \ell_{2}}(z)\right] \mathcal{G}_{\ell_{1} \ell_{2}}^{\lambda}(R ; z) \\
& +R \sum_{\ell_{1}^{\prime}, \ell_{2}^{\prime}} \mathcal{R}_{\ell_{1} \ell_{1}^{\prime} \ell_{2} \ell_{2}^{\prime}}(z) \mathcal{G}_{\ell_{1}^{\prime} \ell_{2}^{\prime}}^{\prime}(R ; z)=0
\end{aligned}
$$

com os coeficientes

$$
\mathcal{P}_{\ell_{1} \ell_{2}}(z)=\frac{2}{1+z^{2}}\left[z+\frac{\left(\ell_{1}+1\right)\left(1-z^{2}\right)}{z}-\frac{1\left(\ell_{2}+1\right) z}{1-z^{2}}\right]
$$




$$
\mathcal{Q}_{\ell_{1} \ell_{2}}(z)=-4\left[\left(\ell_{1}+\ell_{2}+2\right)^{2}+U_{\lambda}(R)\right] /\left(1+z^{2}\right)^{2}+-\sqrt{\frac{\mu_{1}}{\mu}} q_{1} q_{2} \frac{2}{z\left(1+z^{2}\right)} .
$$

Os termos de acoplamento são,

$$
\begin{aligned}
& \mathcal{R}_{\ell_{1} \ell_{1}^{\prime} \ell_{2} \ell_{2}^{\prime}}(z)=\frac{4}{\left(1+z^{2}\right)^{2}}\left(\frac{2 z}{1+z^{2}}\right)^{\ell_{1}^{\prime}-\ell_{1}}\left(\frac{1-z^{2}}{1+z^{2}}\right)^{\ell_{2}^{\prime}-\ell_{2}} \\
& {\left[\frac{m_{1}}{\sqrt{\mu \mu_{1}}} q_{1} q_{3} \Delta_{\ell_{1} \ell_{1}^{\prime} \ell_{2} \ell_{2}^{\prime}}^{+}(z)+\frac{m_{2}}{\sqrt{\mu \mu_{1}}} q_{2} q_{3} \Delta_{\ell_{1} \ell_{1}^{\prime} \ell_{2} \ell_{2}^{\prime}}^{-}(z)\right] .}
\end{aligned}
$$

As expressões para $\Delta^{+}$e $\Delta^{-}$são fornecidas no Apêndice $A$. Os pontos singulares da Eq.(39) ocorrem para $z=\operatorname{tg}\left(\alpha_{ \pm} / 2\right)$, e na Eq.(47) para $z=0, \pm 1, \pm i$. A variável $z$ é limitada por $0 \leq z \leq 1$, de modo que a região de interesse é $\operatorname{Re}(z) \geq 0$. A Eq.(47) deve ser resolvida nos intervalos $\left[0, z_{-}\right],\left[z_{-}, z_{+}\right]$e $\left[z_{+}, 1\right]$, expandindo localmente a função $\mathcal{G}_{\ell_{1} \ell_{2}}^{\lambda}(R ; z)$ em séries de potências. Para que esta expansão seja rapidamente convergente para pequenos valores de $R$, deve-se introduzir o fator $\left(1+z^{2}\right)^{s}$, onde $s=\ell_{1}+\ell_{2}+2-$ $\sqrt{U_{\lambda}(0)}$, e $U_{\lambda}(0)$ é dado pela Eq.(46). A inclusào deste fator faz com que a solução para $R=0$ seja polinomial. Para grandes valores de $R$, de acordo com o tipo de sistema é possivel acrescentar à solução um comportamento exponencial [10]. A forma expandida $\operatorname{de} \mathcal{G}_{\ell_{1} \ell_{2}}^{\lambda}(R ; z) \dot{\mathrm{e}}$

$$
\mathcal{G}_{\ell_{1} \ell_{2}}^{\lambda}(R ; z)=\left(1+z^{2}\right)^{s} e^{-c z R} \sum_{n=0}^{\infty} \mathcal{F}_{\ell_{1} \ell_{2}}^{\lambda}(R ; n) z^{n}
$$

A relação de recorrência que fornece os coeficientes $\mathcal{F}_{\ell_{1} \ell_{2}}^{\lambda}(R ; n)$ pode ser construida algebricamente para evitar os erros associados a operações de ponto flutuante. A importạncia dada a este erro tem origem no grande número de coeficientes inter-relacionados pelo 
método de Frobenius. Esta relação de recorrência é acrescentada a um programa em liguagem FORTRAN e resolvida em um computador VAX em dupla precisão (16 dígitos). Neste programa são controlados dois tipos de erro. Um tem origem no truncamento da expansào (36) em um número máximo de termos $N$ e o outro na inclusão sucessiva de canais de momento angular até um valor máximo $\ell_{\text {max }}$. Assim, os coeficientes podem ser calculados numericamente de modo a gerar uma solıção cuja precisão é controlada. Nos casos em que a expansão da função de onda fica instável devido aos erros de round-off, é possivel utilizar códigos com precisão quádrupla (32 dígitos). Este tipo de problema ocorre para sistemas moleculares onde uma das regiòes, em que sào definidas as expansões, possui dimensôes muito grandes.

O procedimento dos cálculos começa com um valor tentativo para o potencial $U_{\lambda}(R)$ a um ponto fixo de $R$, selecionado pela aplicaçào do método de diferenças e busca de Newton [21] a pontos calculados previamente. Com um ponto escolhido, as relaçóes de recorrência são resolvidas para condiçòes iniciais arbitrárias nas regiões $\left[0, z_{-}\right] \mathrm{e}\left[z_{+}, 1\right]$. Em seguida as equações são resolvidas na região intcrmediária $\left[z_{-}, z_{+}\right]$impondo condições de continuidade simultânea para as soluções independentes em um ponto escolhido $z_{c}$. Deste procedimento obtem-se uma equação determinantal utilizada para corrigir o valor de $U_{\lambda}(R)$. Este processo é repetido até obter a precisào desejada e, em seguida, é reiniciado para um novo valor de $R$.

Uma característica importante na escolha da variável $z$ é que a equação diferencial pode sofrer uma translação sem que perca seu caracter polinomial. Este fato tem duas 
consequências importantes. A primeira é a possibiliclade de propagar a equaçào diferencial na regiào internodiária utilizando sćries de potências, e a seguinte consiste em diminuir o erro na utilizaçào da relação de recorrência através da divisào das regiòes em pequenos intervalos.

As singularidades da Eq.(48) sào mostradas na Figura 1, onde os pólos em \pm 1 e $\pm i$ definem um círculo de convergência de raio unitário com solução regular em $z=0$. As singularidades $z_{+}$e $z_{-}$movem-se no eixo $\operatorname{Re}(z)$ de acordo com as massas do sistema Coulombiano, definindo dois círculos internos nos quais a $\mathrm{Eq}$.(47) deve ser resolvida. Na tabela 1 são mostradas as localizações das singularidades para vários sistemas.

\begin{tabular}{||c|c|c|c|c||}
\hline sistema & $\alpha_{-}$ & $\alpha_{+}$ & $z_{-}$ & $z_{+}$ \\
\hline \hline$H e$ & $\pi / 4$ & $\pi / 2$ & 0.41421 & 1. \\
\hline$d d \mu$ & 1.40677 & $\alpha_{-}$ & 0.84809 & $z_{-}$ \\
\hline$d_{+}^{2}$ & 1.55913 & $\alpha_{-}$ & 0.98840 & $z_{-}$ \\
\hline $\ln S^{4} b$ & 1.31751 & $\pi / 2$ & 0.77411 & 1. \\
\hline$Z n S c$ & 1.18285 & $\pi / 2$ & 0.67162 & 1. \\
\hline$C d S$ & 1.15026 & $\pi / 2$ & 0.64823 & 1. \\
\hline$Z n O$ & 1.14110 & $\pi / 2$ & 0.64174 & 1. \\
\hline$C d S e$ & 1.13222 & $\pi / 2$ & 0.63549 & 1. \\
\hline$p d \mu$ & 1.437 & 1.308 & 0.874 & 0.767 \\
\hline
\end{tabular}

Tabela 1: Localização do pólos para vários sistemas físicos.

Os problemas citados acima para sistemas moleculares como o $d_{2}^{+}$, causados pelas diferenças de massas, são indicados nesta tabela pola posição das singularidades próximas às bordas do raio de convergência.

Os processos de truncanento ficam condicionados também à capacidade de cálculo do computador, sendo portanto importante definir a precisão com que deseja-se obter os 
resultados. Com a resolução da Eq..(47) é possivel resolver a equação radial (13), obtendo as energias c a funçào de onda total do sistema.

\subsection{Diagonalização pelas Fuções de Laguerre-Jacobi}

O tratamento hiperesférico pode ser generalizado para ser aplicado a problemas de quatro corpos com a introdução de um segundo ângulo hiperesférico. A equação radial mantém o mesmo tipo de estrutura, enquanto que a equaçào angular pode ser separada adiabaticamente para cada variável angular. Neste trabalho este tipo de sistema não será estudado, mas é importante observar que a resolução direta das equações acopladas é muito dificil. Esta dificuldade tende a se agravar enormemente para problemas com mais corpos. Um método apropriado para tratar estas equações consiste na expansão das funções angulares em bases que diagonalizem o hamiltoniano. Como foi observado anteriormente, uma base que não leve em conta propriedades do potencial Coulombiano conduz a soluçòes de convergência muito lenta. Nesta seçào é apresentada para sistemas de três corpos, una forma de corrigir este problema, com o intuito de que seja posteriormente generalizada para sistemas de mais corpos. () procedimento utilizanto acui consiste em seguir o tratamento hiperesférico, com a separaçào da funçào de onda em uma parte radial e outra angular, onde $R$ participa parametricamente,

$$
\Phi_{\lambda}(R ; \Omega)=\sum_{\ell_{1} \rho_{2}}(\operatorname{sen} \alpha)^{\ell_{1}+1}(\cos \alpha)^{\ell_{2}+1} \mathcal{Y}_{\ell_{1} \ell_{2}}^{L M}\left(\hat{\xi}_{1}, \hat{\xi}_{2}\right) \mathcal{G}_{\ell_{1} \ell_{2}}^{\lambda}(R ; \alpha)
$$

Os métodos diferem no cálculo de $\mathcal{G}_{\ell_{1} \ell_{2}}^{\lambda}$. Utilizando a variável $z=\operatorname{tg}(\alpha / 2)$, esta função 
será expandida nos autoestados da equação angular (39), na sua forma desacoplada,

$$
\left[\frac{d^{2}}{d z^{2}}+\mathcal{P}_{\ell_{1} \ell_{2}}(z) \frac{d}{d z}+\mathcal{Q}_{\ell_{1} \ell_{2}}(z)\right] \mathcal{G}_{\ell_{1} \ell_{2}}^{\lambda}(R ; z)+R \mathcal{R}_{\ell_{1} \ell_{1} \ell_{2} \ell_{2}}(z) \mathcal{G}_{\ell_{1} \ell_{2}}^{\lambda}(R ; z)=0 .
$$

Para expandir os elementos da base cm séries de potencias repete-se a expressão (36),

$$
\mathcal{G}_{\ell_{1} \rho_{2}}^{\lambda}(R ; z)=\left(1+z^{2}\right)^{s} e^{-c z R} \sum_{n=0}^{\infty} \mathcal{F}_{\ell_{1} \rho_{2}}^{\lambda}(R ; n) z^{n}
$$

Utilizando esta base, que contém informaçòes do potencial, fica garantida a convergência do procedimento de diagonalização, especialnuente nos limites de $R \rightarrow 0 \mathrm{e} \infty . \Lambda$ solução numérica das equações angulares desacopladas é rapidamente convergente com relação a expansào da funçào de onda cun séries de potencias. Lsta propriedade pode tornar o procedimento descrito apropriado para tratar também de sistemas moleculares. 


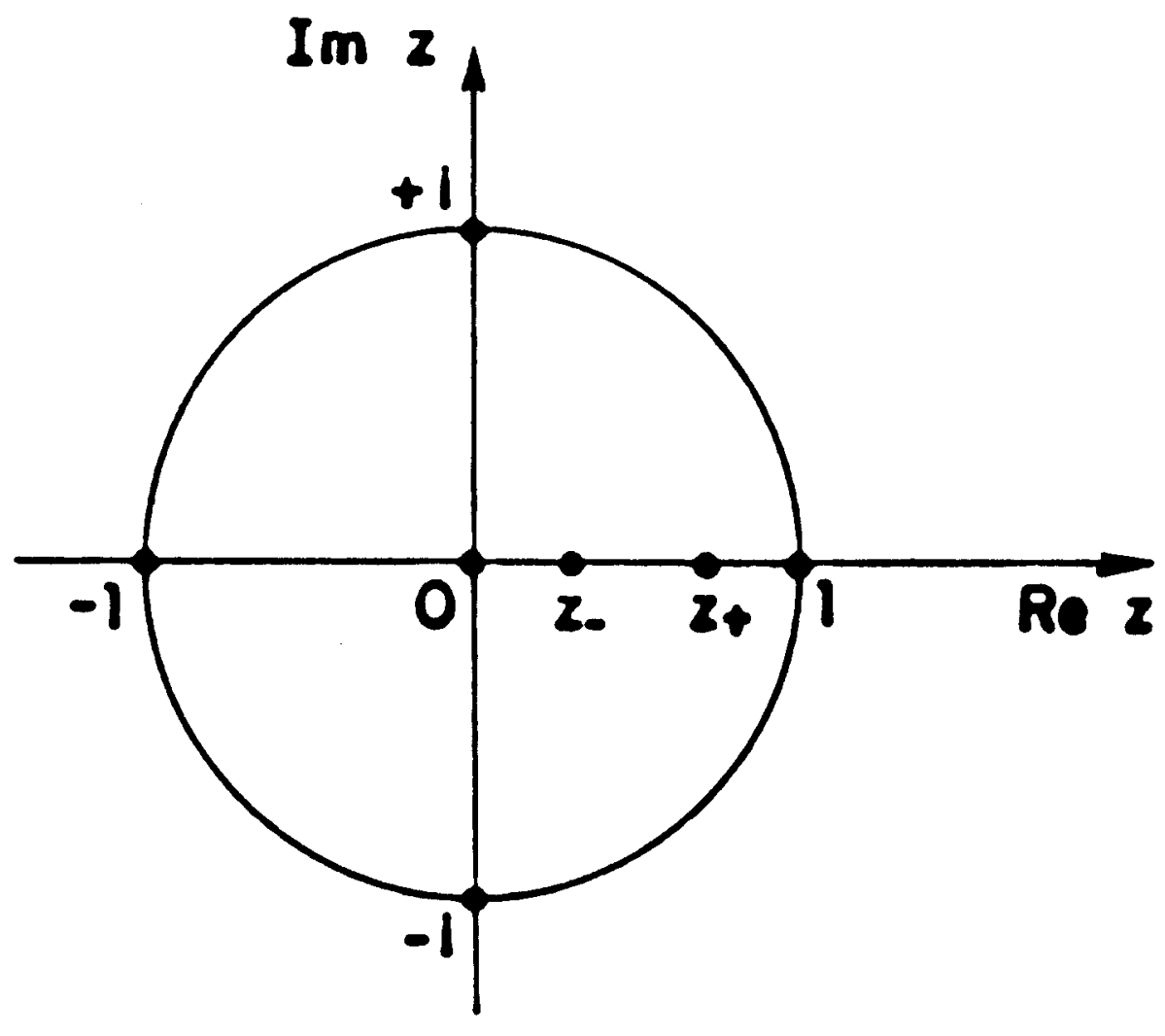

Figura 1: Pontos singulares da equação angular na variável $z$. 
Capítulo 4

Aplicações 
A seguir serão discutidos vários sistemas Coulombianos de três corpos. As características de cada um estão relacionadas com a localização de $z_{-}$e $z_{+}$, de acordo com as massas das partículas. As curva de potencial e funções de canal serão obtidas para $L=0 \rightarrow \ell_{1}=\ell_{2}=\ell$, por fornecer o estado fundamental, além de permitir o estudo de estados autoionizantess.

\section{1 Átomo de Hélio}

No átomo de hélio o núcleo de carga $Z e$, possui massa muito maior que os elétrons sendo portanto considerado fixo. Assim, a equação diferencial (2) é transformada fazendo $m_{1} \rightarrow \infty$ e $m_{2}=m_{3}=m_{c}$, o que fornece o potencial hiperesférico,

$$
\hat{V}(\alpha, 0)=-\frac{Z e^{2}}{\operatorname{sen} \alpha}-\frac{Z e^{2}}{\cos \alpha}+\frac{e^{2}}{\sqrt{1-\operatorname{sen}(2 \alpha) \cos \theta}} .
$$

Na figura 2 observa-se para esse potencial os pólos atrativos elétron-núcleo para $\alpha=0$, $\pi / 2$ c repulsivo clétron-elétron en $(0, \alpha)=(0, \pi / 1)$. As regiòns de expantsào da função de: onda são $(0 \leq \alpha \leq \pi / 4)$ e $(\pi / 4 \leq \alpha \leq \pi / 2)$. A solução para a segunda região é obtida fazendo $\alpha \rightarrow \pi / 2-\alpha$. Esta troca não muda a forma da equação diferencial, de modo que basta resolver o sistema de equações na primeira região utilizando a condição do princípio de exclusão de Pauli,

$$
\Phi_{\lambda}\left(R ; \alpha, \Omega_{1}, \Omega_{2}\right)=(-1)^{S} \Phi_{\lambda}\left(R ; \pi / 2-\alpha, \Omega_{2}, \Omega_{1}\right) .
$$

O índice $S$ representa o spin, e a troca $r_{1} \rightarrow r_{2}$ transforma $\alpha$ em $\pi / 2-\alpha$. A função $\mathcal{Y}_{\ell_{1} \ell_{2}}^{L M}$ 
sofre a transformação,

$$
\mathcal{Y}_{\ell_{1} \ell_{2}}^{L M}\left(\Omega_{1}, \Omega_{2}\right)=(-1)^{\ell_{1}+\ell_{2}+L} \mathcal{Y}_{\ell_{2} \ell_{1}}^{L M}\left(\Omega_{2}, \Omega_{1}\right)
$$

Finalmente obtem-se as propriedades de $\mathcal{G}_{\ell_{1} \ell_{2}}^{\lambda}$ :

$$
\mathcal{G}_{\ell_{1} \ell_{2}}^{\lambda}(R ; \alpha)=(-1)^{\ell_{1}+\ell_{2}+l+s} \mathcal{G}_{\ell_{2} \ell_{1}}^{\lambda}(R ; \pi / 2-\alpha)
$$

Estas relações devem ser satisfeitas para todos valores de $\alpha$, em particular em $\alpha=\pi / 4$, obtendo

$$
\mathcal{G}_{\ell_{1} \ell_{2}}^{\lambda}(R ; \pi / 4)=(-1)^{\ell_{1}+\ell_{2}+L+S} \mathcal{G}_{\ell_{2} \ell_{1}}^{\lambda}(R ; \pi / 4)
$$

e, derivando esta expressão,

$$
\frac{d}{d \alpha}{ }_{\pi / 4} \mathcal{G}_{\ell_{1} \ell_{2}}^{\lambda}(R ; \alpha)=(-1)^{\ell_{1}+\ell_{2}+L+S+1} \frac{d}{d \alpha}{ }_{\pi / 4} \mathcal{G}_{\ell_{2} \ell_{1}}^{\lambda}(R ; \alpha)
$$

Para obter o conjunto de soluções que incluam a curva do estado fundamental, escolhe-se para as aplicaçòes $L=0$ e $S=0$. Com isso, $\ell_{1}=\ell_{2}$ impondo a simetria das funções angulares com relaçäo a $\alpha=\pi / 4$. $\Lambda$ forma simétrica da funçüo $\mathcal{G}_{\ell_{1} \ell_{2}}^{\lambda}$ pode ser olservada na Figura 3. Para $R=0$ a interaçào é anulada c a funçào de onda se comporta como uma partícula confinada entre $\alpha=0$ e $\alpha=\pi / 2$. Com a inclusão de $R$ a densidade de probabilidade começa a localizar na proximidade dos pólos atrativos. No limite de grandes valores de $R$ este comportamento fica claro fazendo uma expansão da equação de autovalores do operador (8),

$$
\left[\frac{d^{2}}{d \alpha^{2}}-\ell_{1}\left(\ell_{1}+1\right)\left(\frac{1}{\alpha^{2}}+\frac{1}{3}+\frac{\alpha^{2}}{15}+\ldots\right)-\ell_{2}\left(\ell_{2}+1\right)\left(1+\alpha^{2}+\ldots\right)+4 Z R\left(\frac{1}{\alpha}+\frac{\alpha}{6}+\frac{7 \alpha^{2}}{360}+\ldots\right)\right.
$$




$$
\left.+4 Z R\left(1+\frac{\alpha^{2}}{2}+\ldots\right)-U_{\lambda}\right] \mathcal{G}_{\ell_{1} \ell_{2}}^{\lambda}=2 R \sum_{\ell_{1}^{\prime} \ell_{2}^{\prime} J}\left(1+\frac{\alpha^{2}}{2}+\ldots\right)(\alpha+\ldots)^{J} C_{\ell_{1} \ell_{2} \ell_{1}^{\prime} \ell_{2}^{\prime}}^{J} \mathcal{G}_{\ell_{1}^{\prime} \ell_{2}^{\prime}}^{\lambda}
$$

Esta equação pode ser reescrita mudando para a variável $\rho=\alpha R$ e dividindo a expressão resultante por $R^{2}$ para obter,

$$
\left[\frac{d^{2}}{d \rho^{2}}-\frac{\ell_{1}\left(\ell_{1}+1\right)}{\rho^{2}}+\frac{2 Z}{\rho}+\frac{2(Z-1)}{R}-U_{\lambda}+\mathcal{O}\left(\frac{1}{R^{2}}\right)\right] \mathcal{G}_{\ell_{1} \ell_{2}}^{\lambda}=\sum_{\ell_{1}^{\prime} \ell_{2}^{\prime} J} \mathcal{O}\left(\frac{1}{R^{2}}\right) \mathcal{G}_{\ell_{1}^{\prime} \ell_{2}^{\prime}}^{\lambda} .
$$

No limite de $R \rightarrow \infty$ esta equaçào assume a forma da equação de autovalores do átomo de hidrogênio com um núcleo de carga $Z e$. Desta forma é possível determinar o valor da curva de potencial neste limite como sendo,

$$
\frac{U_{\lambda}}{R^{2}}=-\frac{Z^{2}}{n_{\lambda}^{2}}-\frac{2(Z-1)}{R}+\frac{U^{(2)}}{R^{2}}+\frac{U^{(3)}}{R^{3}}+\ldots
$$

onde $n_{\lambda}^{\ell}=1,2,3, \ldots U^{(2)}$ e $U^{(3)}$ devem ser determinados numericamente. Esta relação indica que esta é uma região de dissociação, onde um dos elétrons forma um estado ligado com o núcleo, enquanto que o outro está espallhado. A forma correta da funçào angular é do tipo hidrogenóide, com decaimento exponencial dependente de $R e^{-Z R \alpha / n_{\lambda}}$ obtido da solução da Eq.(63), como pode ser observado na Figura 3. Nesta figura é desenhada a forma da funçâo $\left|(\operatorname{sen} \alpha)^{\ell_{1}+1}(\cos \alpha)^{\ell_{2}+1} \mathcal{G}_{\ell_{1} \ell_{2}}^{\lambda}\right|^{2}$ para o estado fundamental, em termos das variáveis $R$ e $\alpha$. As curvas de potencial dos três primeiros estados angulares são plotadas na Figura 4 e classificadas por ordem crescente, de acordo com a Tabela 2. Estas curvas foram obtidas com dez canais de momento angular acoplados. As duas primeiras curvas excitadas tendem ao limite de ionização $n_{\lambda}=2$. A mais baixa, $\operatorname{com} \ell=1$ e $n=0$ assume neste limite o estado 2p hidrogenóide (Fig.5), e a seguinte, com $\ell=0$, o estado 2s. Para 
esta última são observados (Fig.6) dois nós na origem de $R$ devido a $n=2$.

\begin{tabular}{||c|c|c|c|c||}
\hline curva & $\ell$ & $n$ & $n_{\lambda}$ & $-U_{\lambda}(0)$ \\
\hline \hline 0 & 0 & 0 & 1 & 4 \\
\hline 1 & 1 & 0 & 2 & 16 \\
\hline 2 & 0 & 2 & 2 & 36 \\
\hline 3 & 2 & 0 & 3 & 36 \\
\hline 4 & 1 & 2 & 3 & 64 \\
\hline 5 & 3 & 0 & 3 & 64 \\
\hline 6 & 0 & 4 & 4 & 100 \\
\hline 7 & 2 & 2 & 4 & 100 \\
\hline
\end{tabular}

Tabela 2: Classificação das curvas de potencial para $L=0 \operatorname{com} \ell_{1}=\ell_{2}=\ell$.

A energia do estado fundamental foi obtida para as aproximações adiabáticas citadas no Capítulo 2, acoplando até três soluções angulares. Os resultados na tabela 3 indicam a grande precisão que o método permite, em comparação com valores variacionais.

\begin{tabular}{||l|l||}
\hline método & Energia \\
\hline \hline EAA & -2.92967 \\
\hline UAA & -2.895 \\
\hline CAA (2 curvas) & -2.89862 \\
\hline CAA (3 curvas) & -2.90372 \\
\hline Variacional $[22]$ & -2.903724377 \\
\hline
\end{tabular}

Tabela 9: Energias do estado fundamental do átomo de hélio

As curvas de potencial e funções de canal foram obtidas até o valor de $R=10$, suficiente para a convergência da energia do estado fundamental. Para o calculo dos estados excitados e ressonâncias é necessário valores maiores de $R$. Para exemplificar estes estados de modo qualitativo obteve-se as soluções angulares até $R=\mathbf{4 0}$ com quatro canais de momento angular acoplados, e a solução radial na aproximação adiabática extrema. As 
energias mais baixas suportadas pelas três primeiras curvas estão representas nas figuras 7, 8 e 9, e nas figuras 10,11 e 12 as densidades de probabilidade radiais correspondentes. Nota-se que as funções de onda radial para cada curva sào semelhantes em forma, mas ficam mais dispersas quanto mais excitada a curva de potencial. Para obter a função de onda total multiplica-se as funçòes angulares pelas radiais. A densidade de probabilidade para os estados excitados e ressonantes pode ser vista nas figuras (14-19).

Para $R$ pequeno e $R$ grande a correlação entre as curvas é pequena, permitindo uma rápida convergência com respeito a $N$ e $\ell_{\max }$. A regiào crítica com relação à convergência se localiza em torno do ponto de mínimo de $U_{\lambda}(R) / R^{2}$. Na Tabela 4 são fornecidos estes valores para $\ell_{\max }=1, \ldots, 30$ e $N=70,90,130 . A$ escollia de $N$ e $\ell_{\max }$ na obtenção das curvas de potencial deve ser feita de acordo com a precisão descjada e também com o tempo de computaçào, que é aproximadamente proporcional à $N \ell_{m u x}^{3}$, podendo rapidamente saturar o computador. Uma opsào para resolver este problema é a utilização de máquinas mais rápidas, como os computadores CRAY ou CONVEX, ou através da paralelização do algoritmo em computadores do tipo $\Lambda \mathrm{CP}$. 


\begin{tabular}{||c|c|c|c||}
\hline $\operatorname{lmax} N$ & 70 & 90 & 130 \\
\hline \hline 2 & -8.48231553 & -8.48231553 & -8.48231553 \\
\hline 3 & -8.489208655 & $-8.1892086(5)$ & -8.4892086 .5 \\
\hline 4 & -8.49095792 & -8.490955792 & -8.49095792 \\
\hline 5 & -8.49159146 & -8.49159146 & -8.49159146 \\
\hline 6 & -8.49187364 & -8.49187364 & -8.49187364 \\
\hline 7 & -8.49201767 & -8.49201767 & -8.49201767 \\
\hline 8 & -8.49209866 & -8.49209866 & -8.49209866 \\
\hline 9 & -8.49214763 & -8.49214763 & -8.49214763 \\
\hline 10 & -8.49217894 & -8.49217894 & -8.49217894 \\
\hline 11 & -8.49219989 & -8.49219989 & -8.49219989 \\
\hline 12 & -8.49221443 & -8.49221443 & -8.49221443 \\
\hline 13 & -8.49222483 & -8.49222483 & -8.49222483 \\
\hline 14 & -8.49223247 & -8.49223246 & -8.49223246 \\
\hline 15 & -8.49223820 & -8.49223819 & -8.49223819 \\
\hline 16 & -8.49224259 & -8.49224259 & -8.49224259 \\
\hline 17 & -8.49224601 & -8.49224599 & -8.49224599 \\
\hline 18 & -8.49224873 & -8.49224869 & -8.49224869 \\
\hline 19 & -8.49225096 & -8.49225084 & -8.49225084 \\
\hline 20 & -8.49225286 & -8.49225259 & -8.49225259 \\
\hline 21 & -8.49225456 & -8.49225418 & -8.49225402 \\
\hline 22 & -8.49225615 & -8.49225520 & -8.49225520 \\
\hline 23 & -8.49225763 & -8.49225619 & -8.49225618 \\
\hline 24 & -8.49225898 & -8.49225702 & -8.49225700 \\
\hline 25 & -8.49226010 & -8.49225774 & -8.49225770 \\
\hline 26 & -8.492260993 & -8.49225839 & -8.492259830 \\
\hline 27 & -8.49226146 & -8.49225898 & -8.49225881 \\
\hline 28 & -8.49226174 & -8.49225955 & -8.49225925 \\
\hline 29 & -8.49226187 & -8.49226011 & -8.49225963 \\
\hline 30 & -8.49226190 & -8.49226066 & -8.49225996 \\
\hline & & & \\
\hline
\end{tabular}

Tabela 4: Valores calculados de $U(R) / R^{2}$ (cm a.u.) para a curva mais baixa do $H_{e}$ no seu ponto de mínimo, em funçào do $N$ e ? ma.r. 


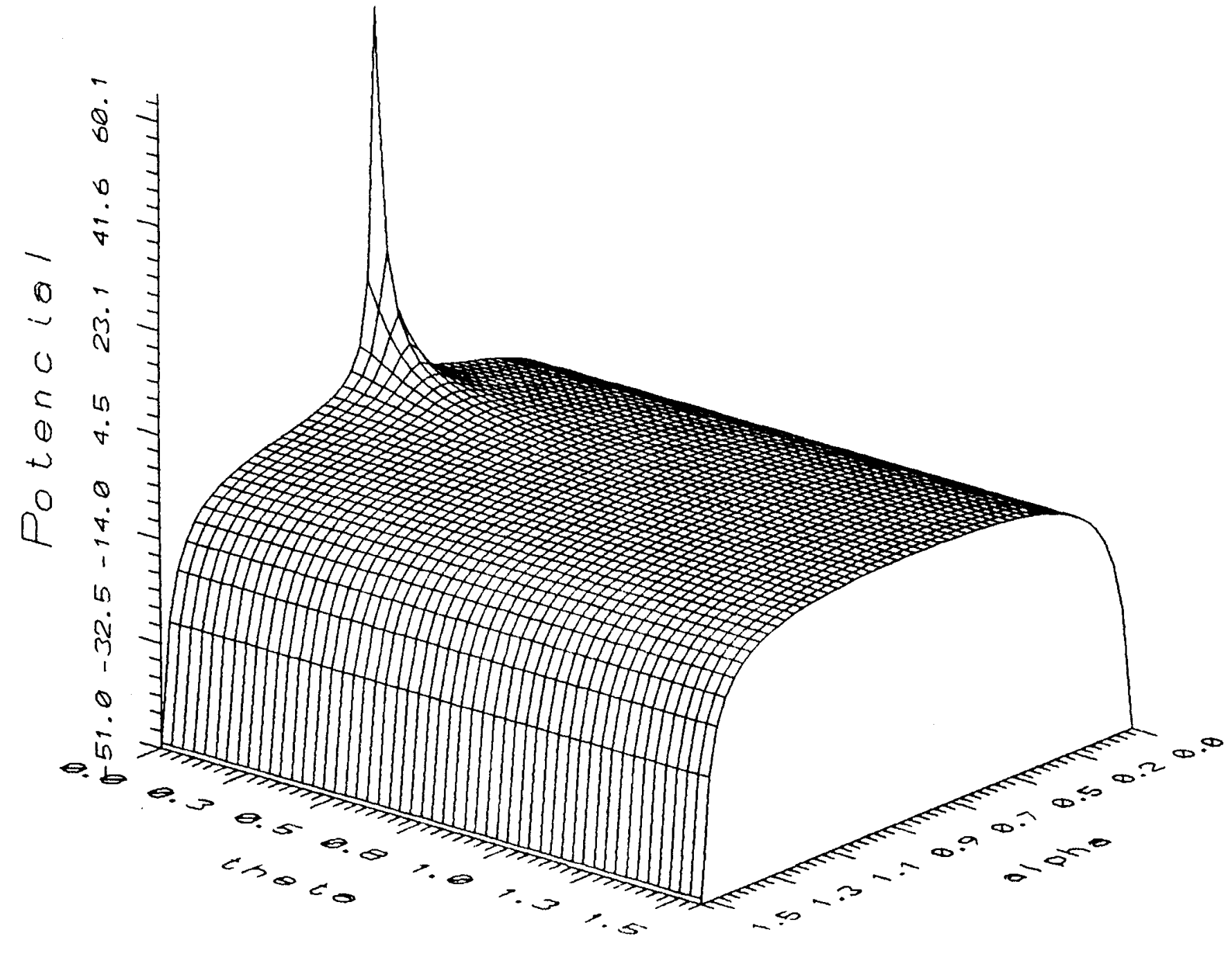

Figura 2: Potencial hiperesférico do átomo de hélio. 


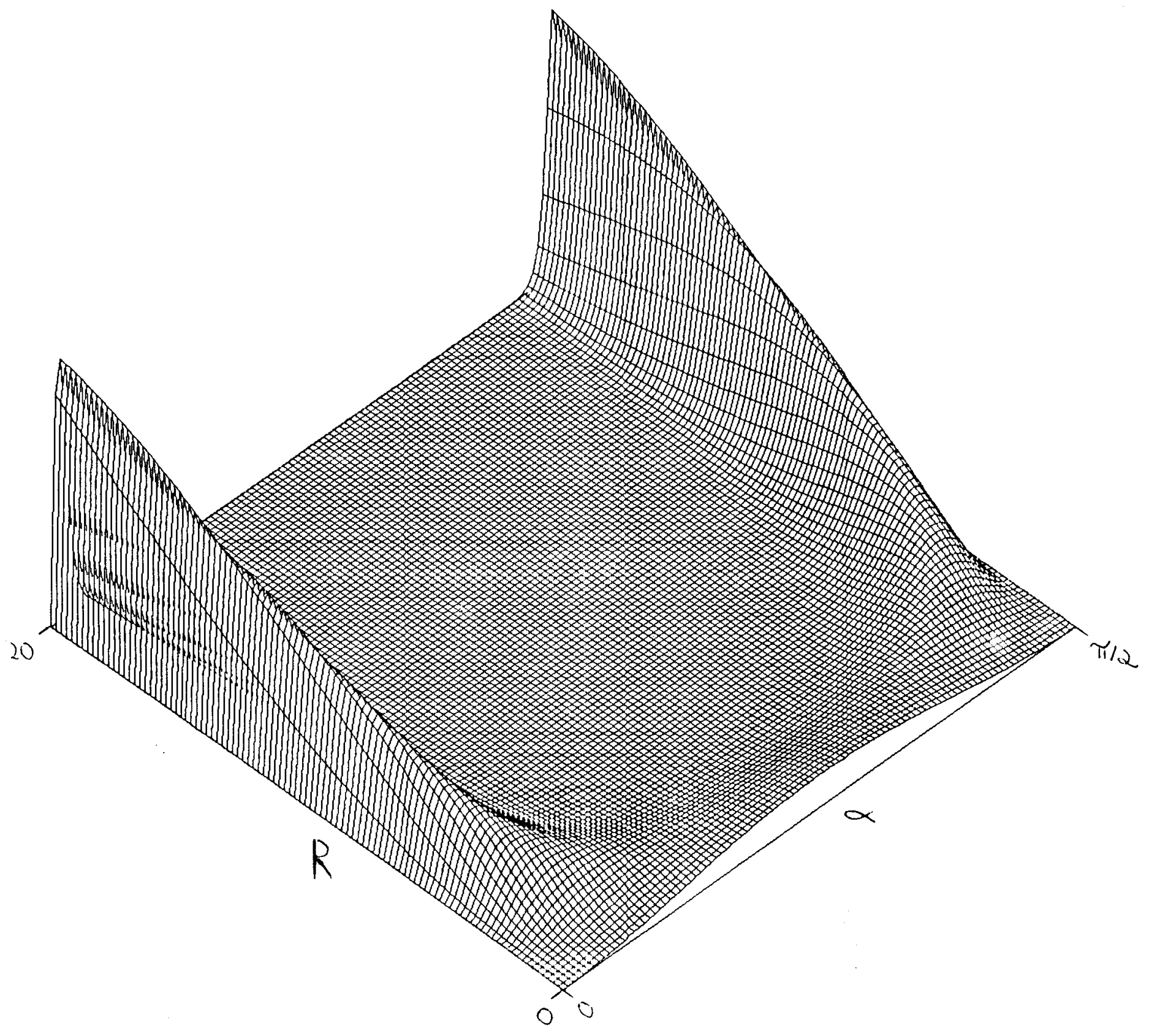

Figura 3: Densidade de probabilidade da soluçio angular 0. 


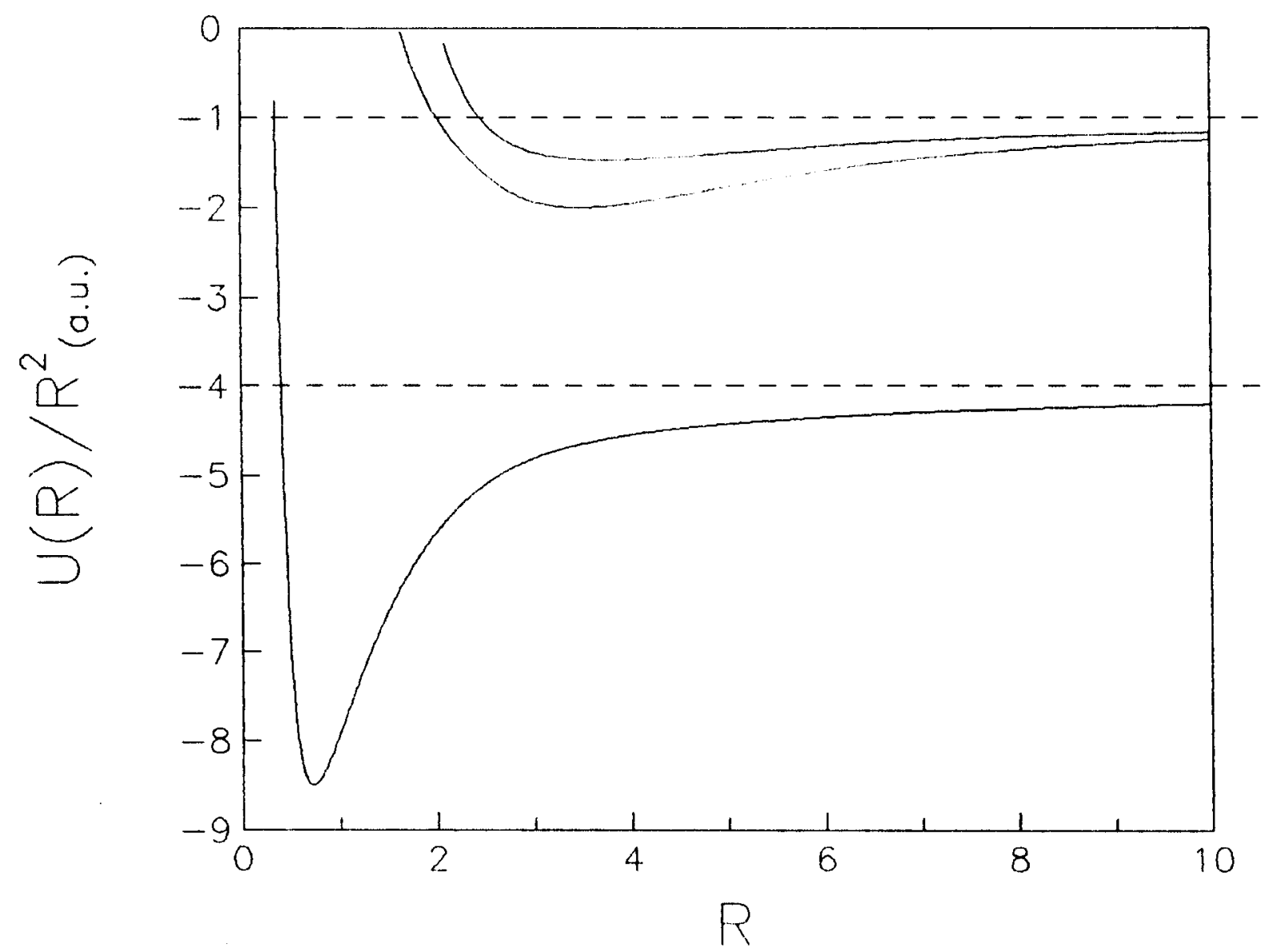

Figura 1: Curvas de potencial mais baixas para o atomo de hélio. 


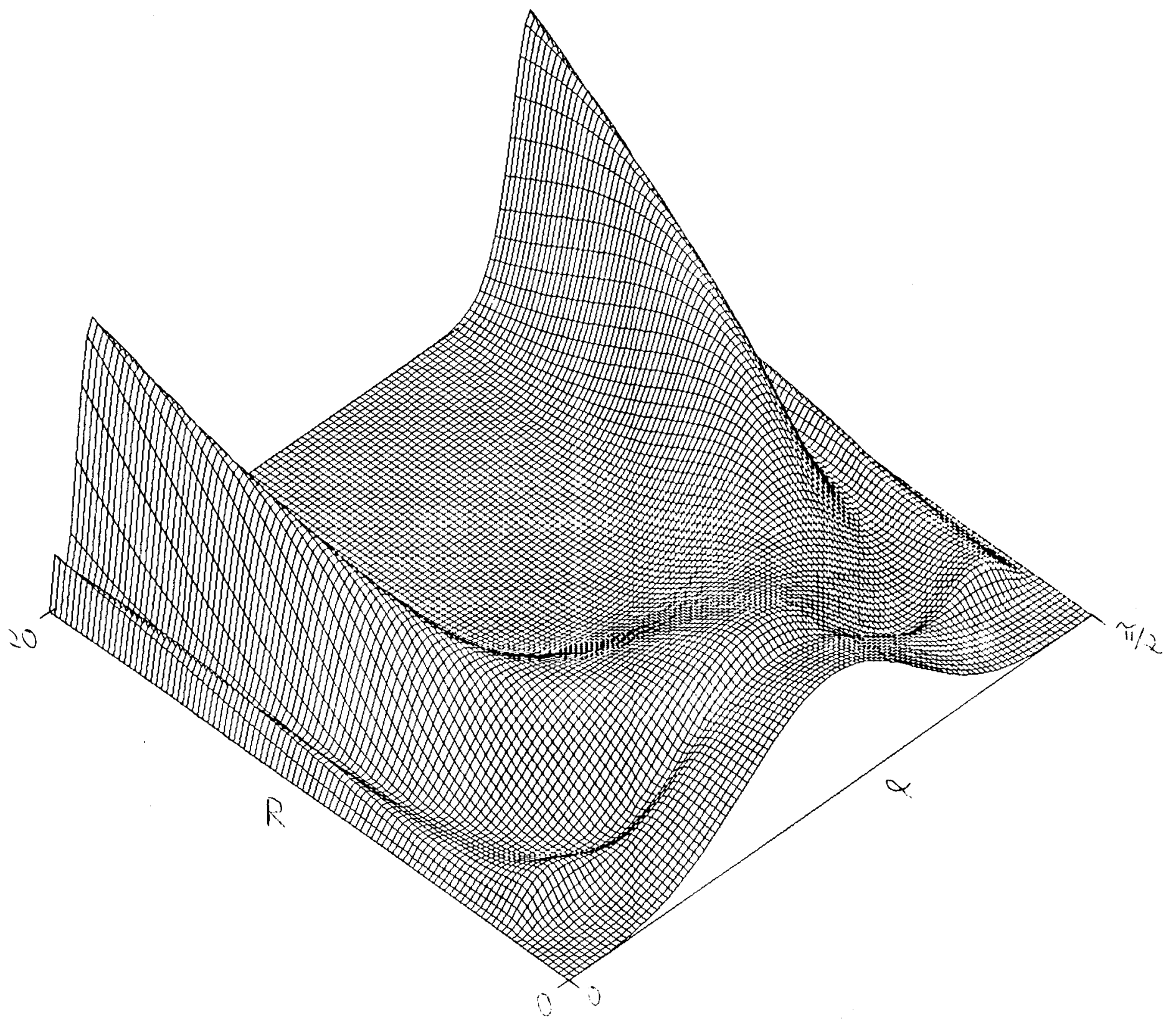

Figura 5: Densidade de probabilidade da soluçào angular 1. 


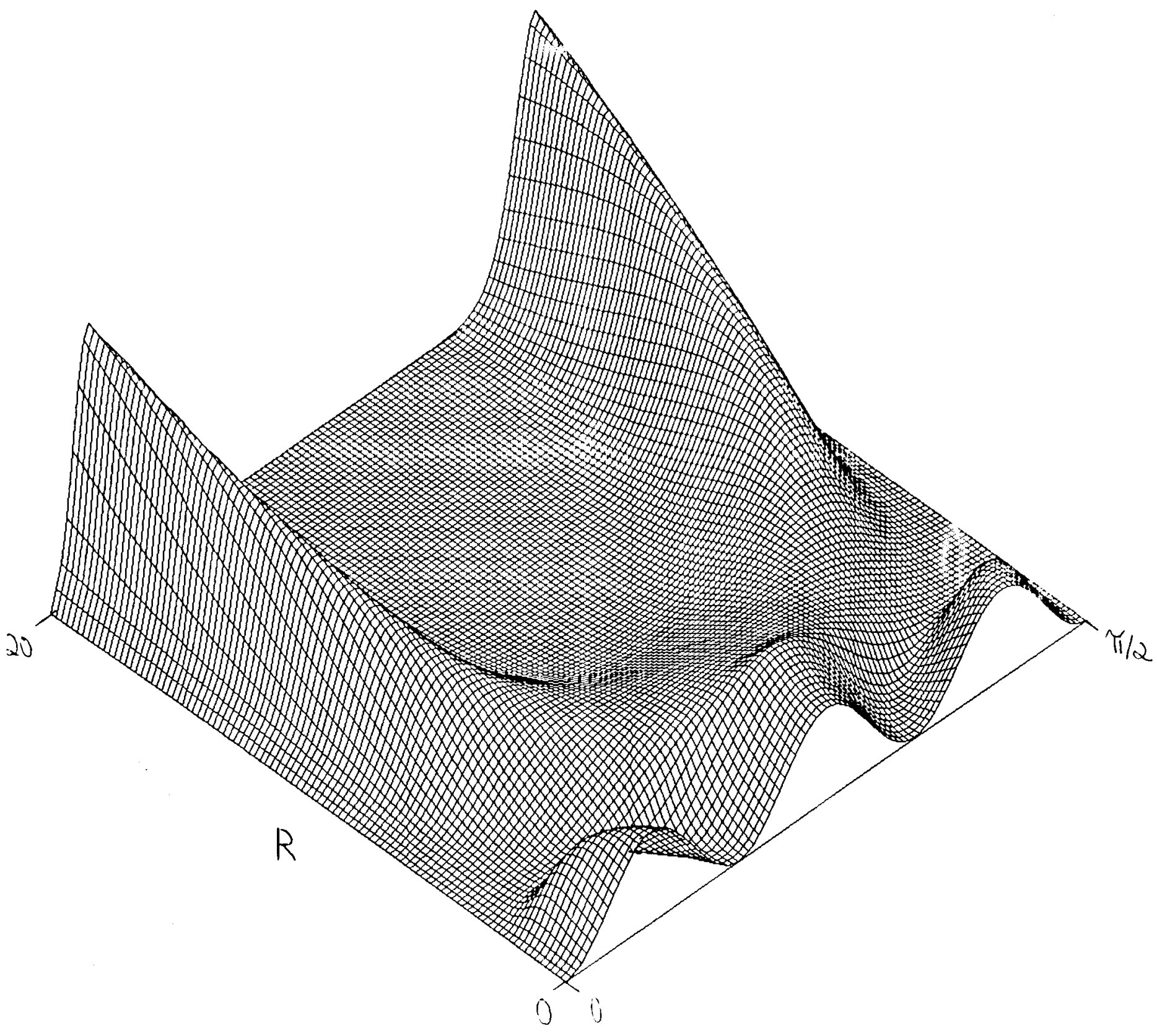

Figura 6: Densidade de probabilidade da soluçào angular 2. 


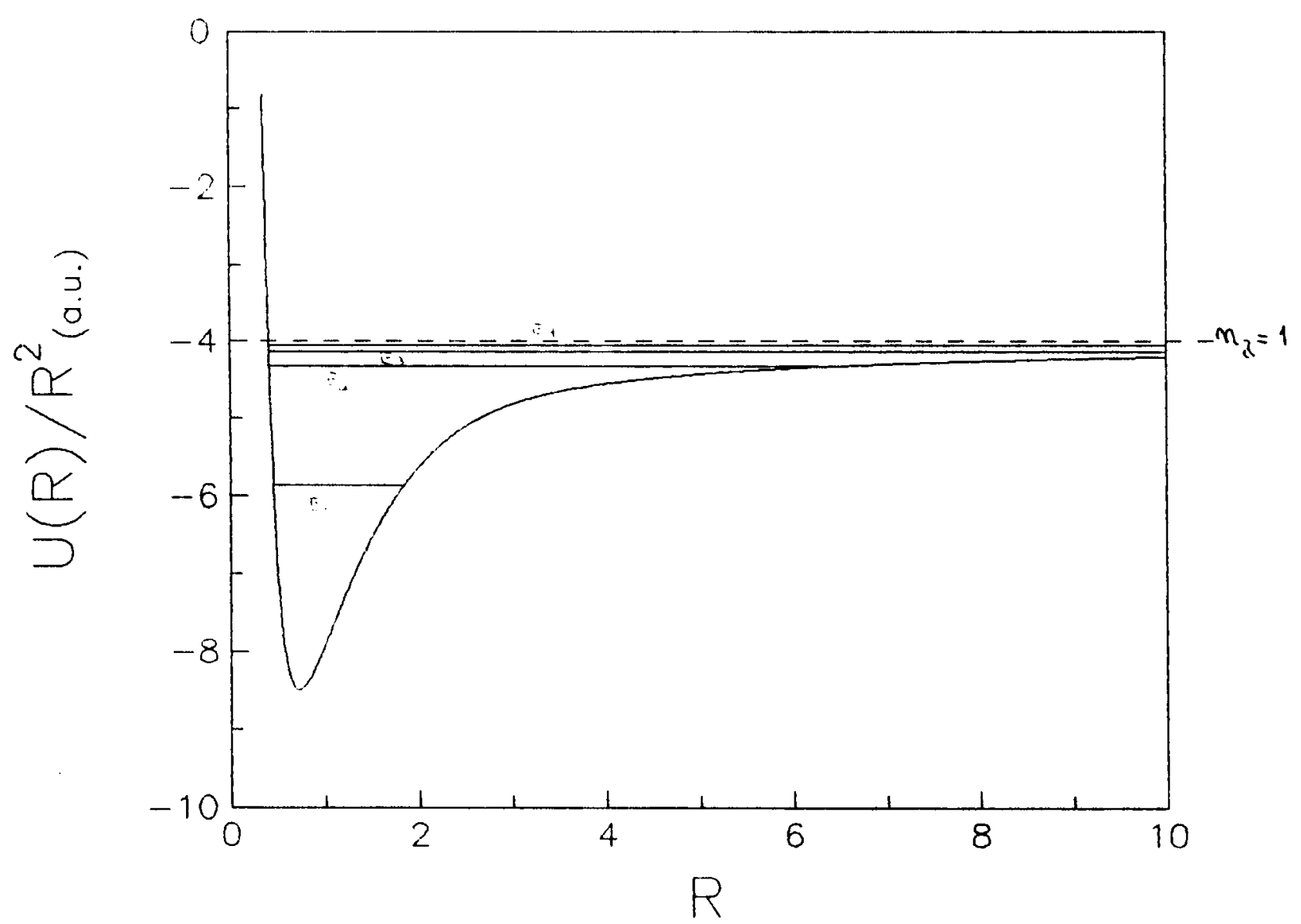

Figura 7: Curva de potencial mais baixa do átomo de hélio com o estado lundamental e as primeinass concrgias excitarlas. 


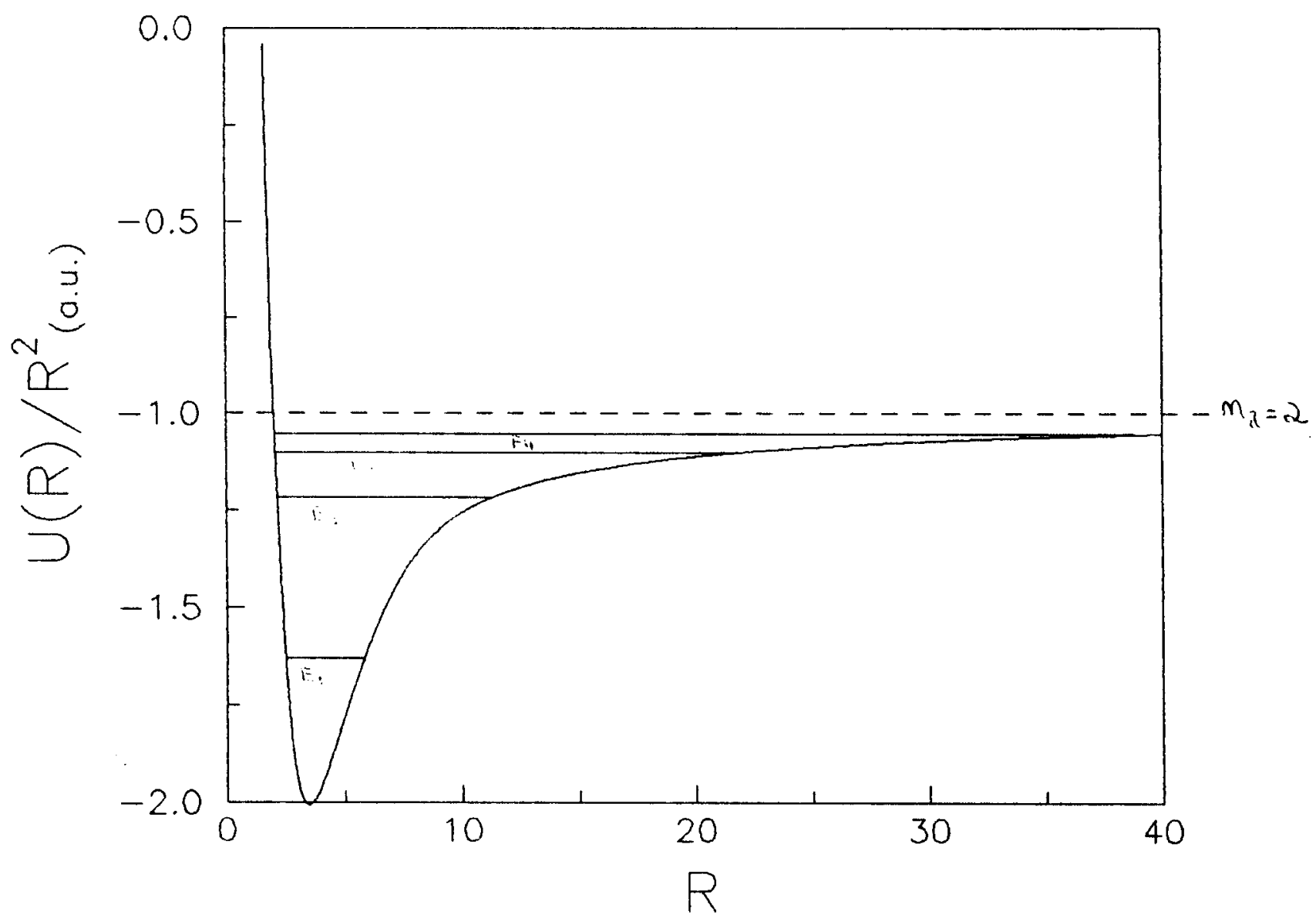

Figura 8: Primeira curva de potencial excitada do átomo de hélio com as primeiras ressonânucias. 


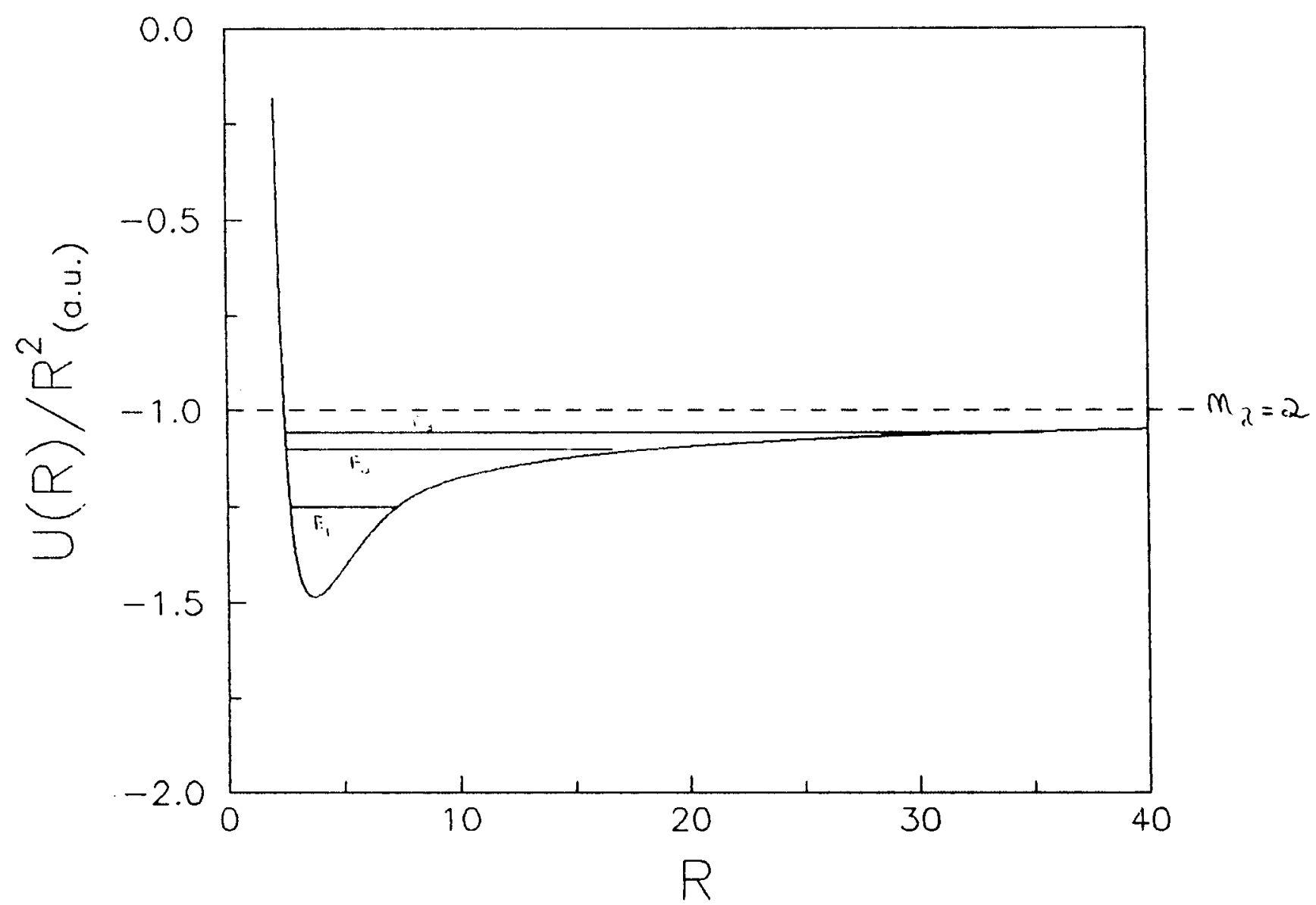

Figura 9: Segunda curva de potencial excitada do átomo de hélio com as primeiras ressontuncials. 


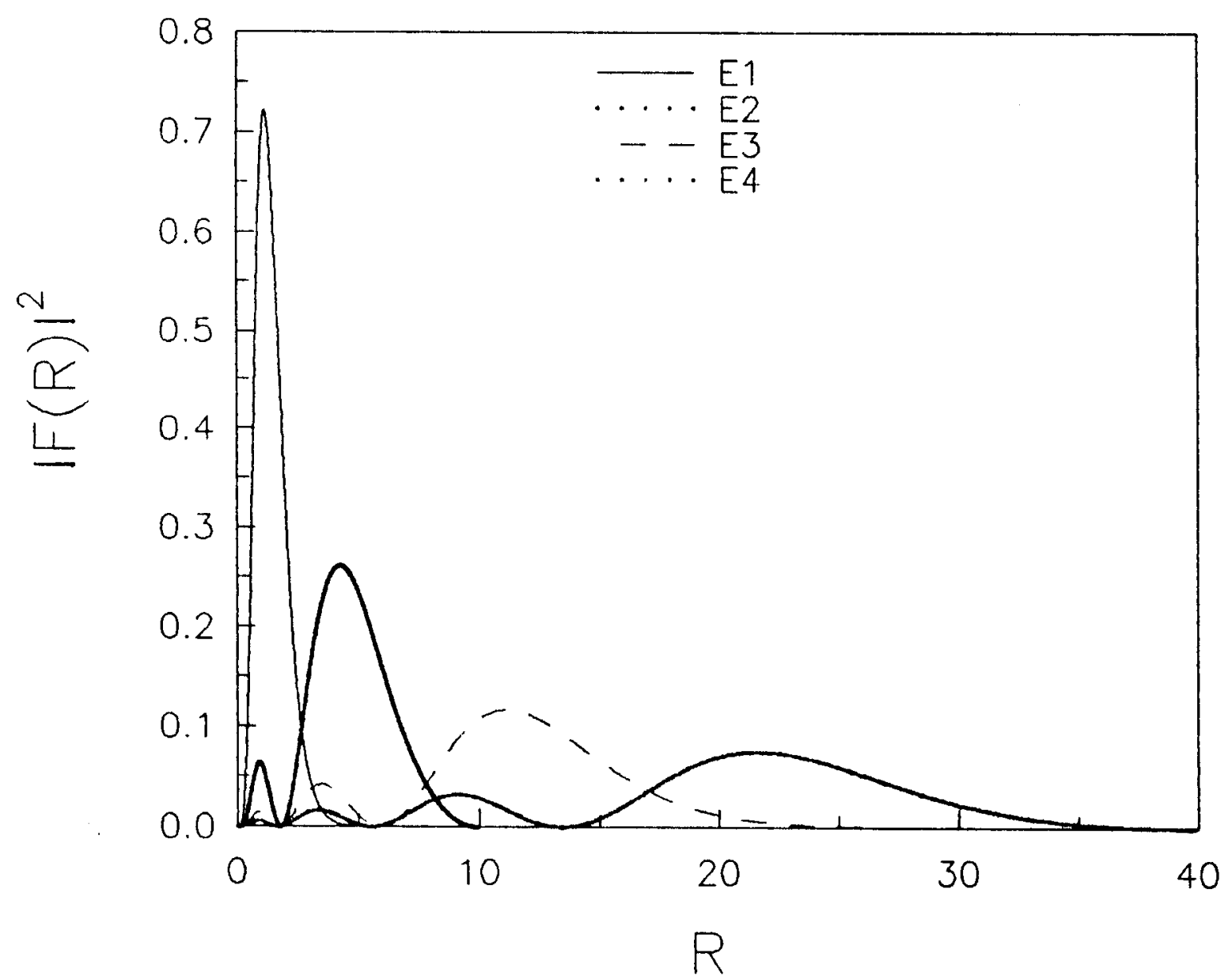

Figura 10: Densidade de probabilidade radial correspondente às energias da figura 7. 


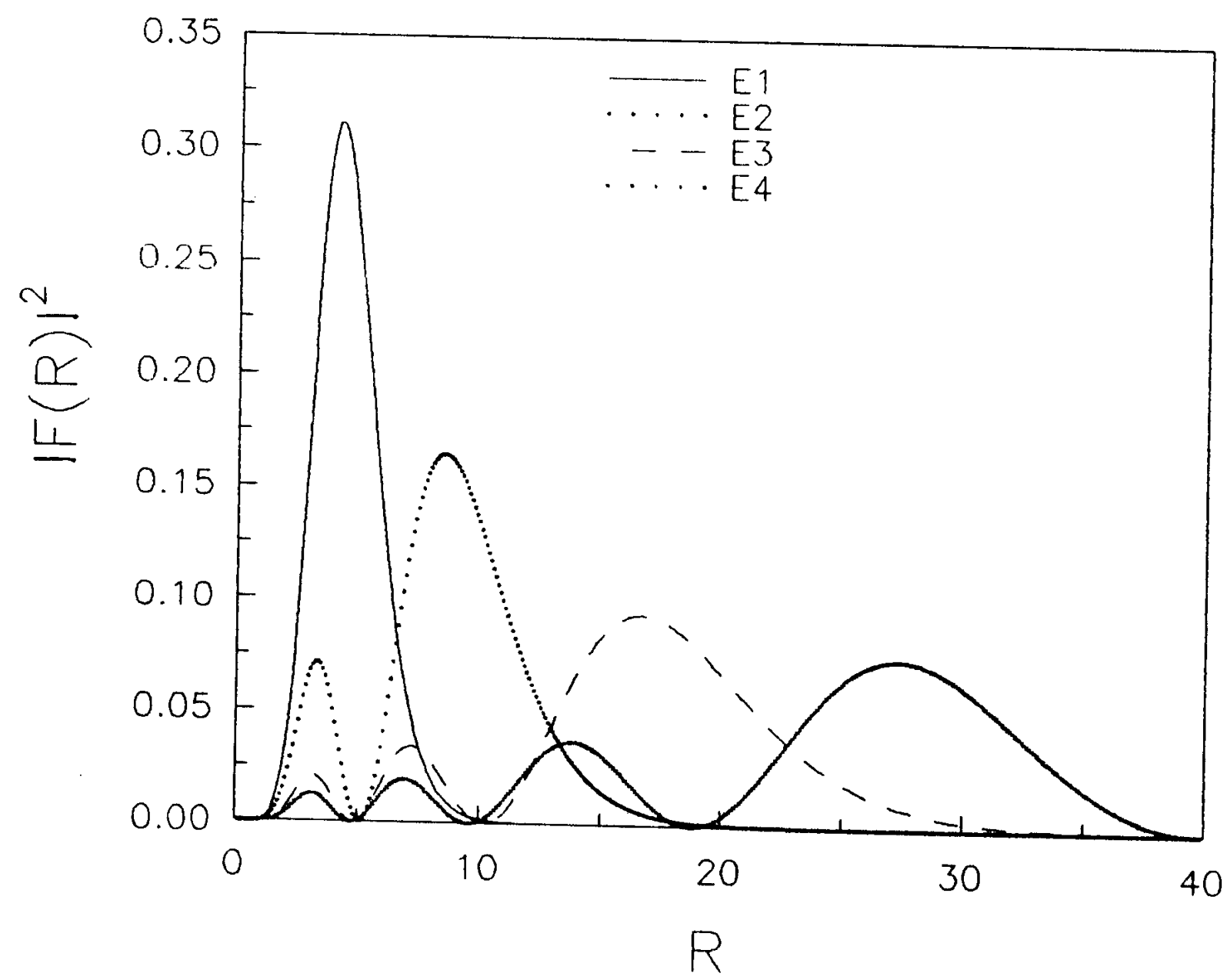

Figura 11: Densidade de probabilidade radial correspondente às energias da figura 8 . 


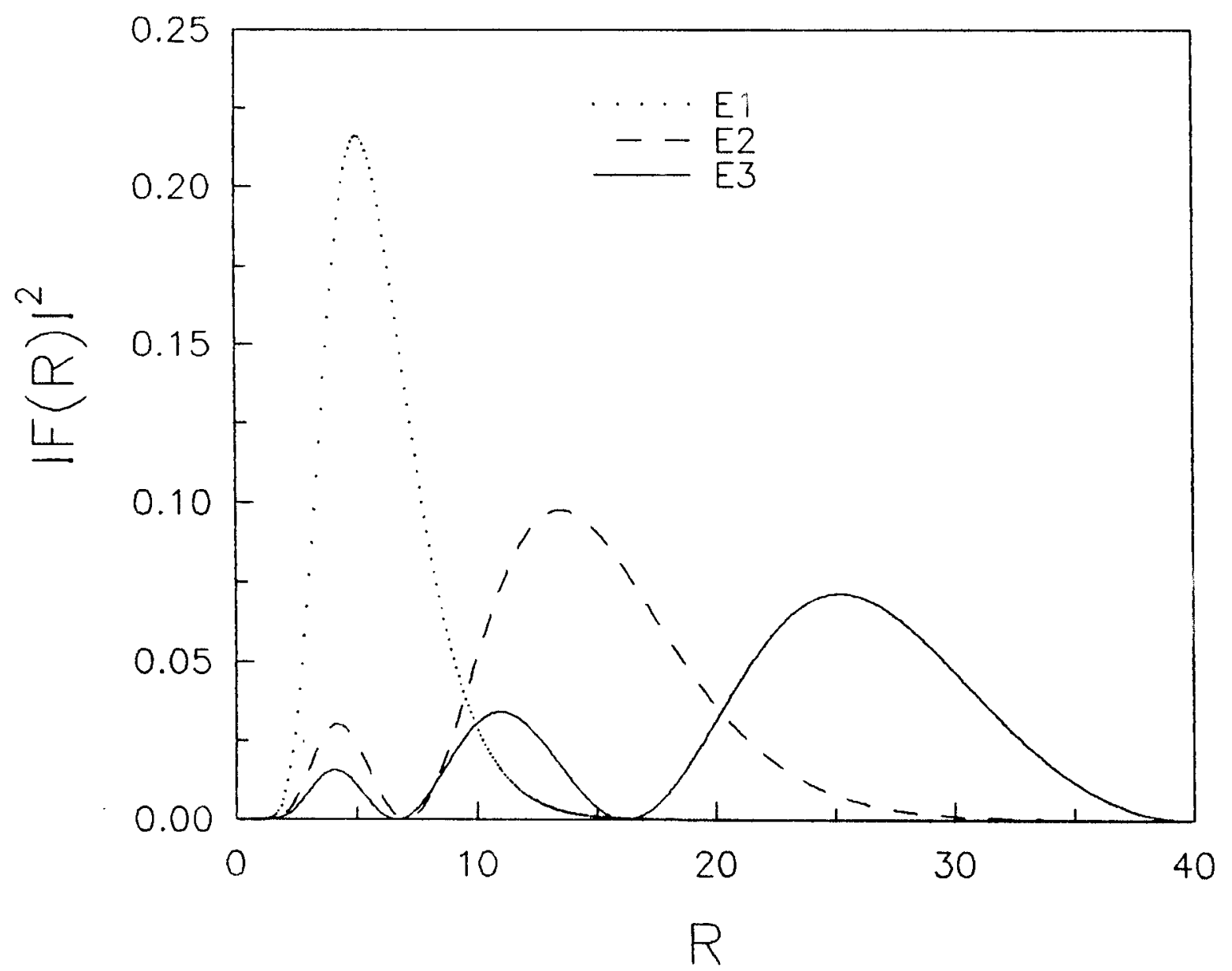

Figura 12: Densidade de probabilidade radial correspondente às energias da figura 9. 


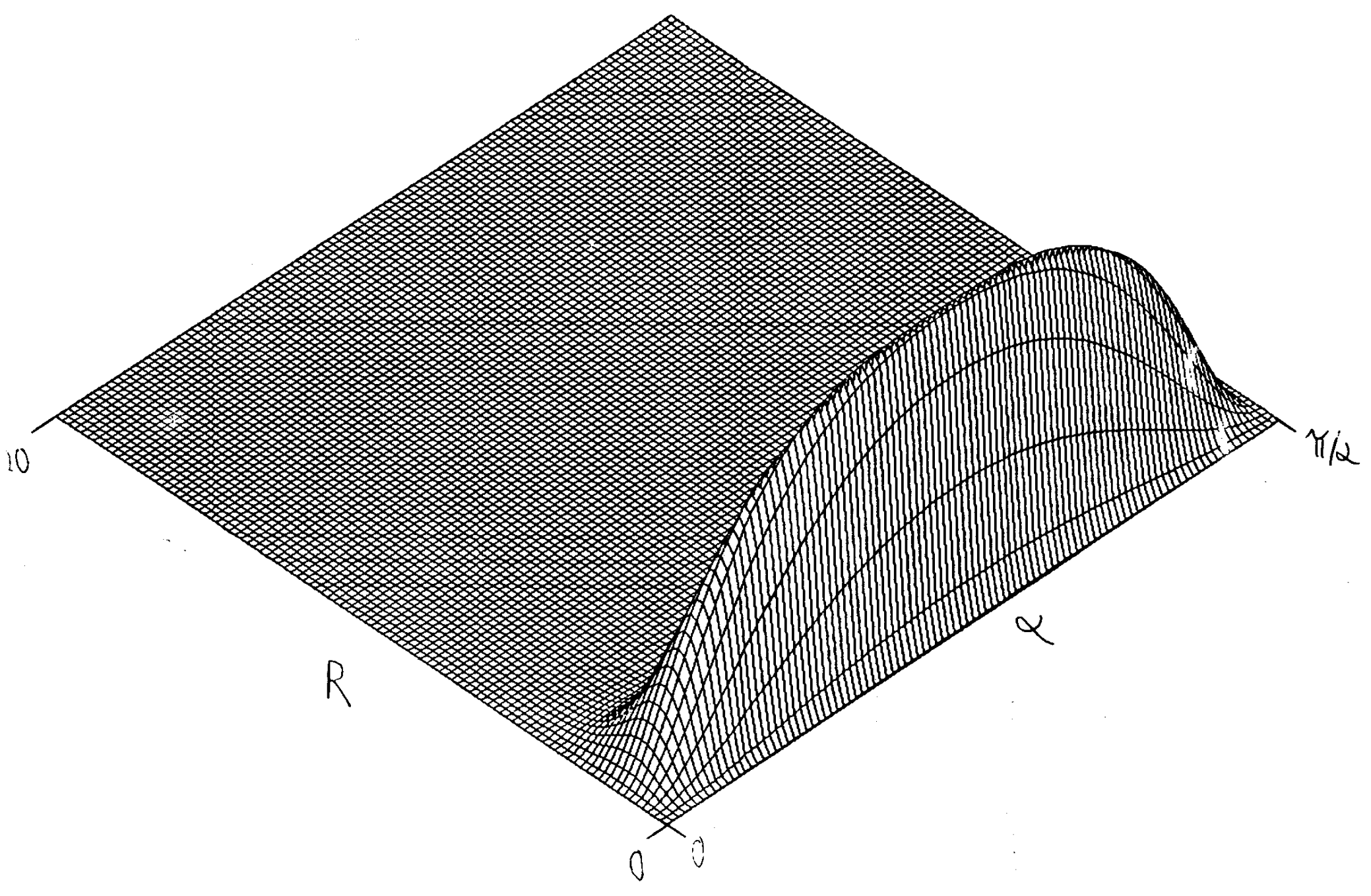

Figura 13: Densidade de probabilidade da funçào angular total correspondente a energia do estado fundannental. 


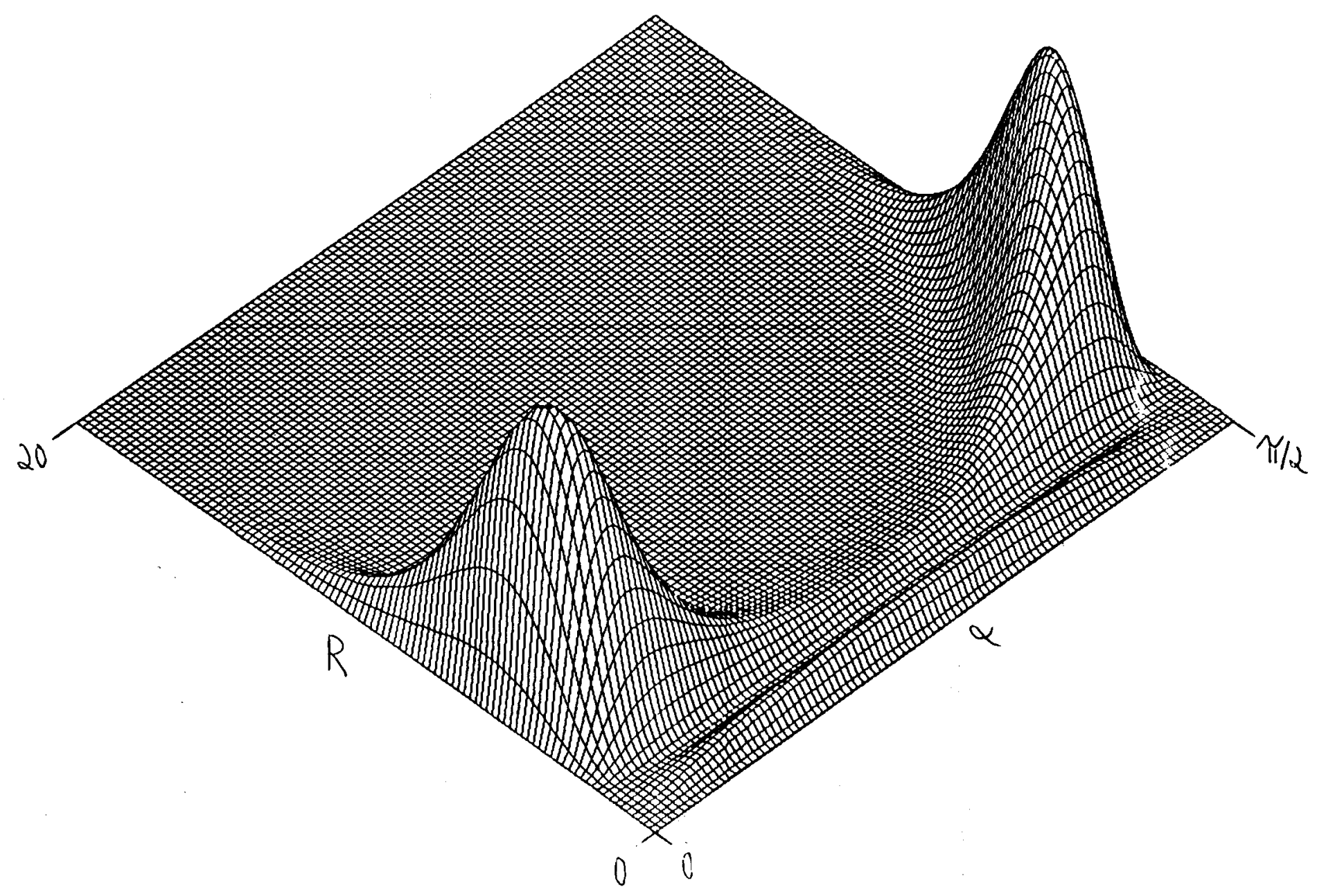

Figura 14: Densidade de probabilidade da lunçào angular total correspondente a segunda energia da curva 0 . 


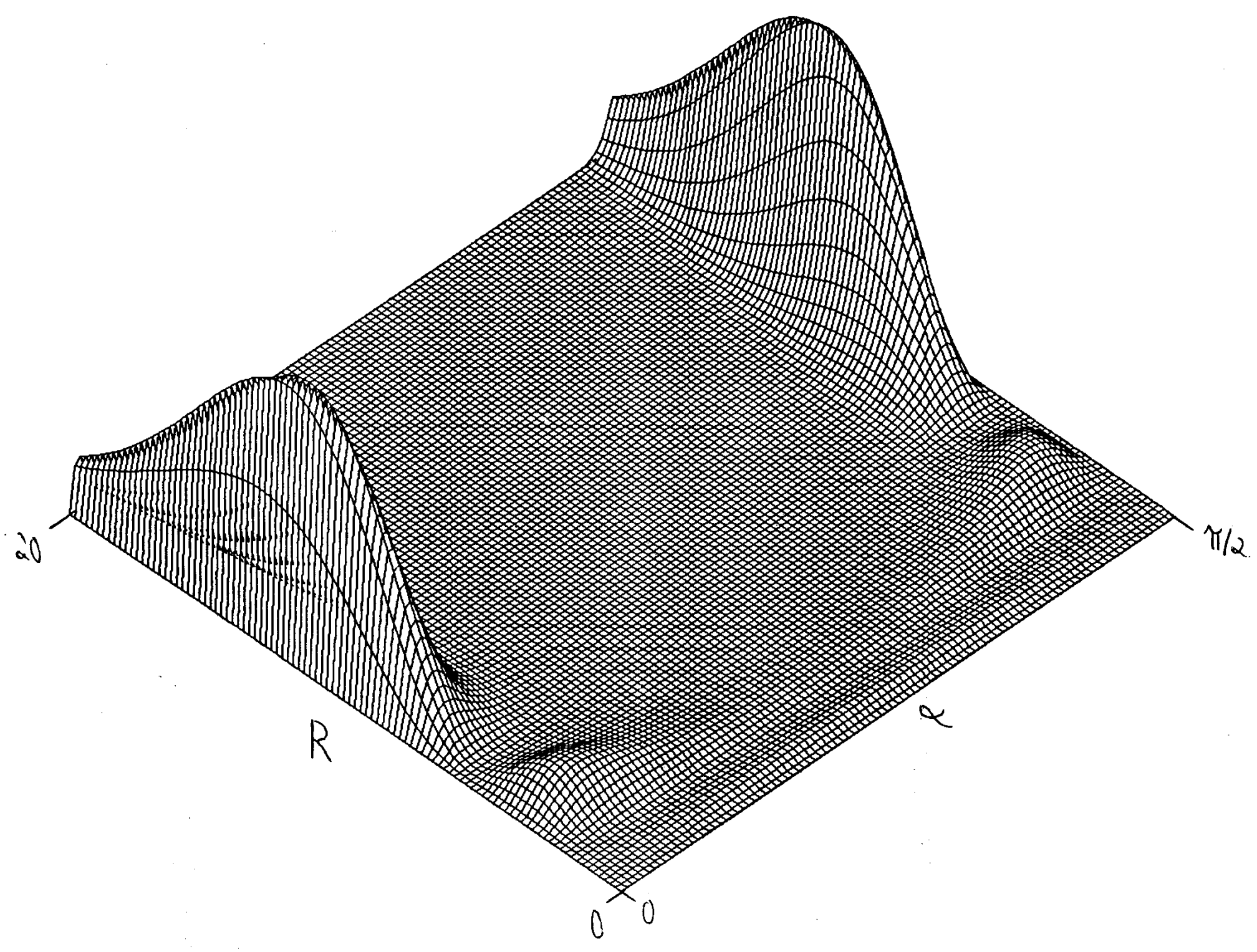

Figura 15: Densidade de probabilidade da funçào angular total correspondente a terceira energia da curva 0 . 


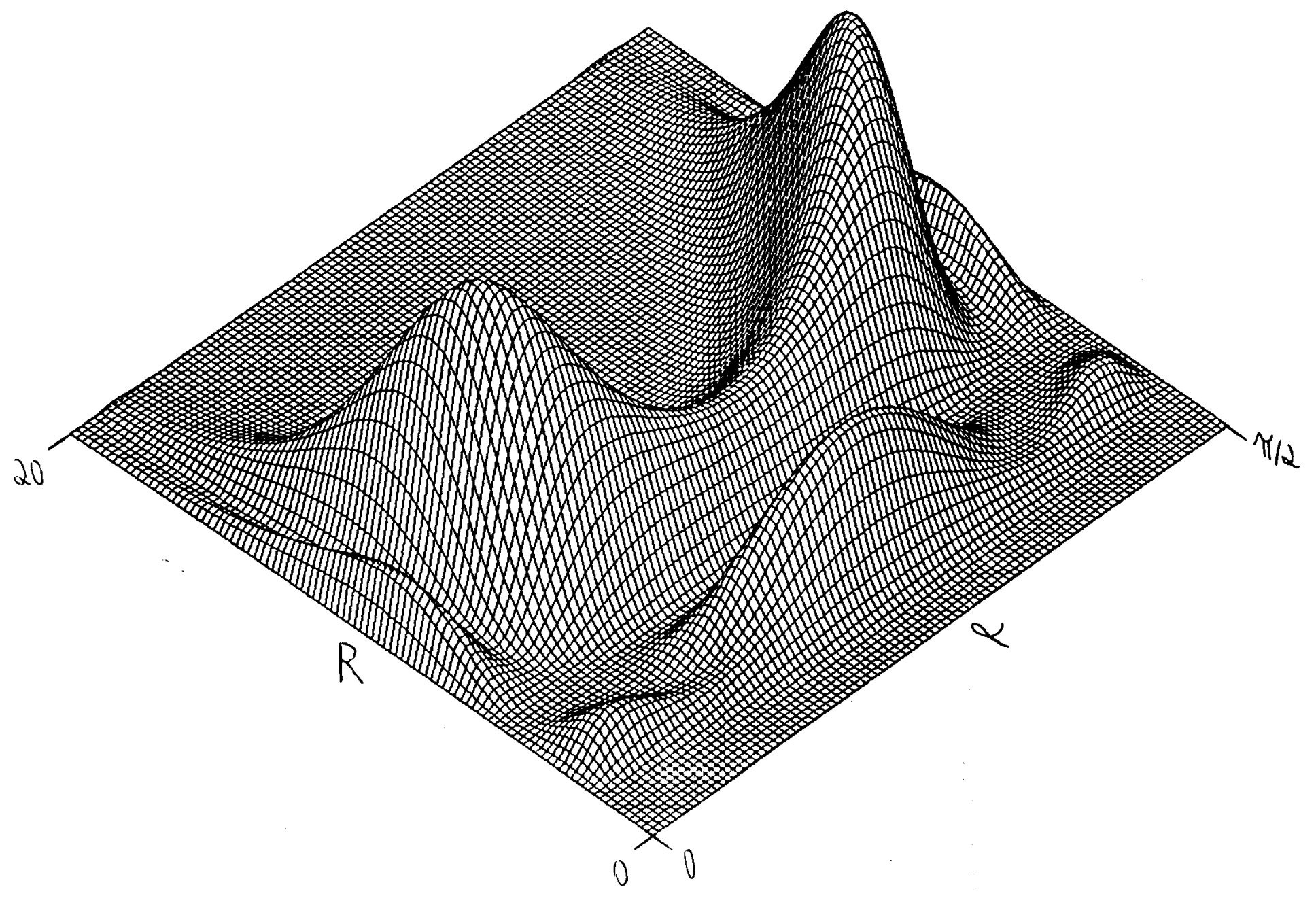

Figura 17: Densidade de probabilidade da função angular total correspondente a segunda energia da curva 1 . 


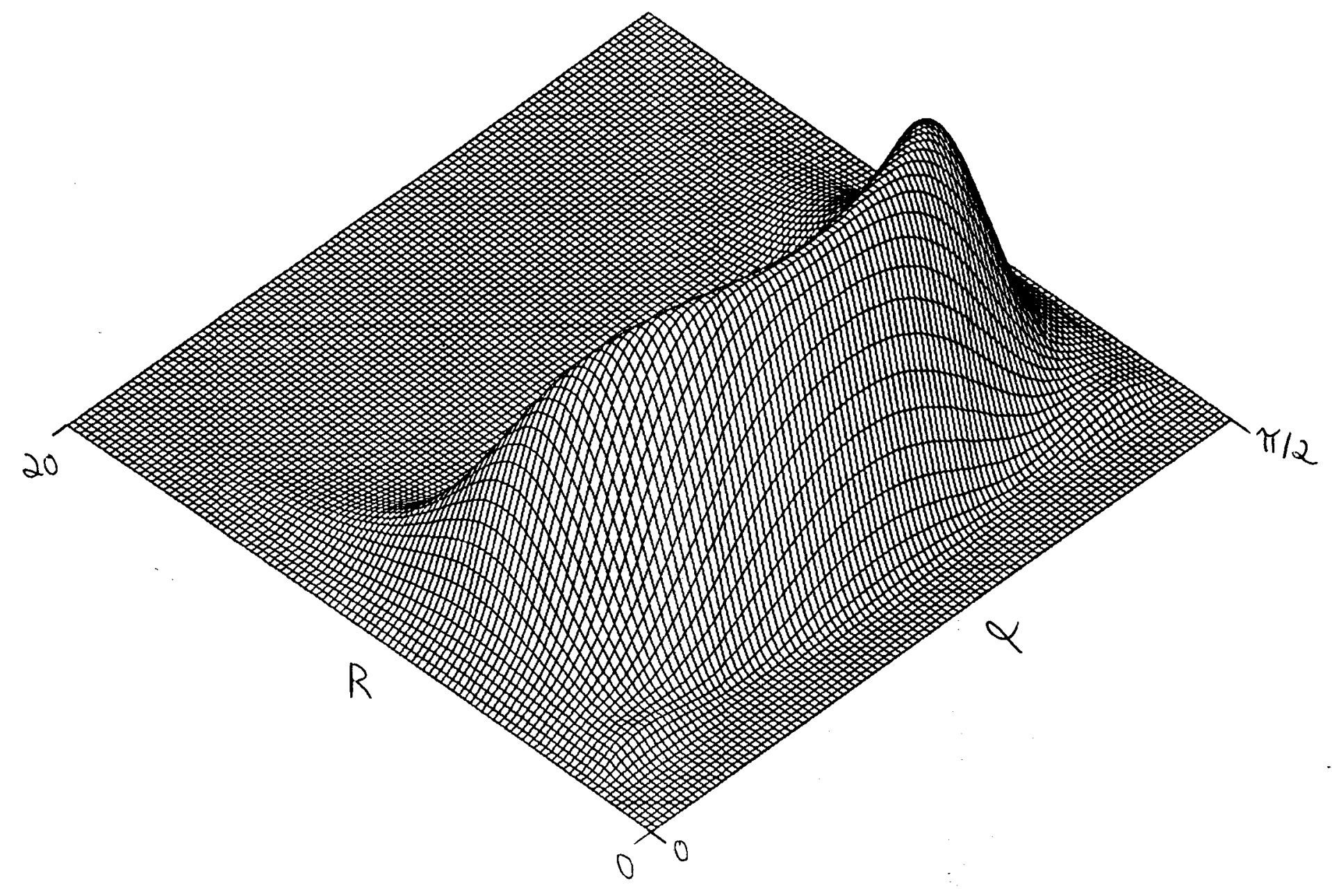

Figura 18: Densidade de probabilidade da funçào angular total correspondente a primeira energia da curva 2. 


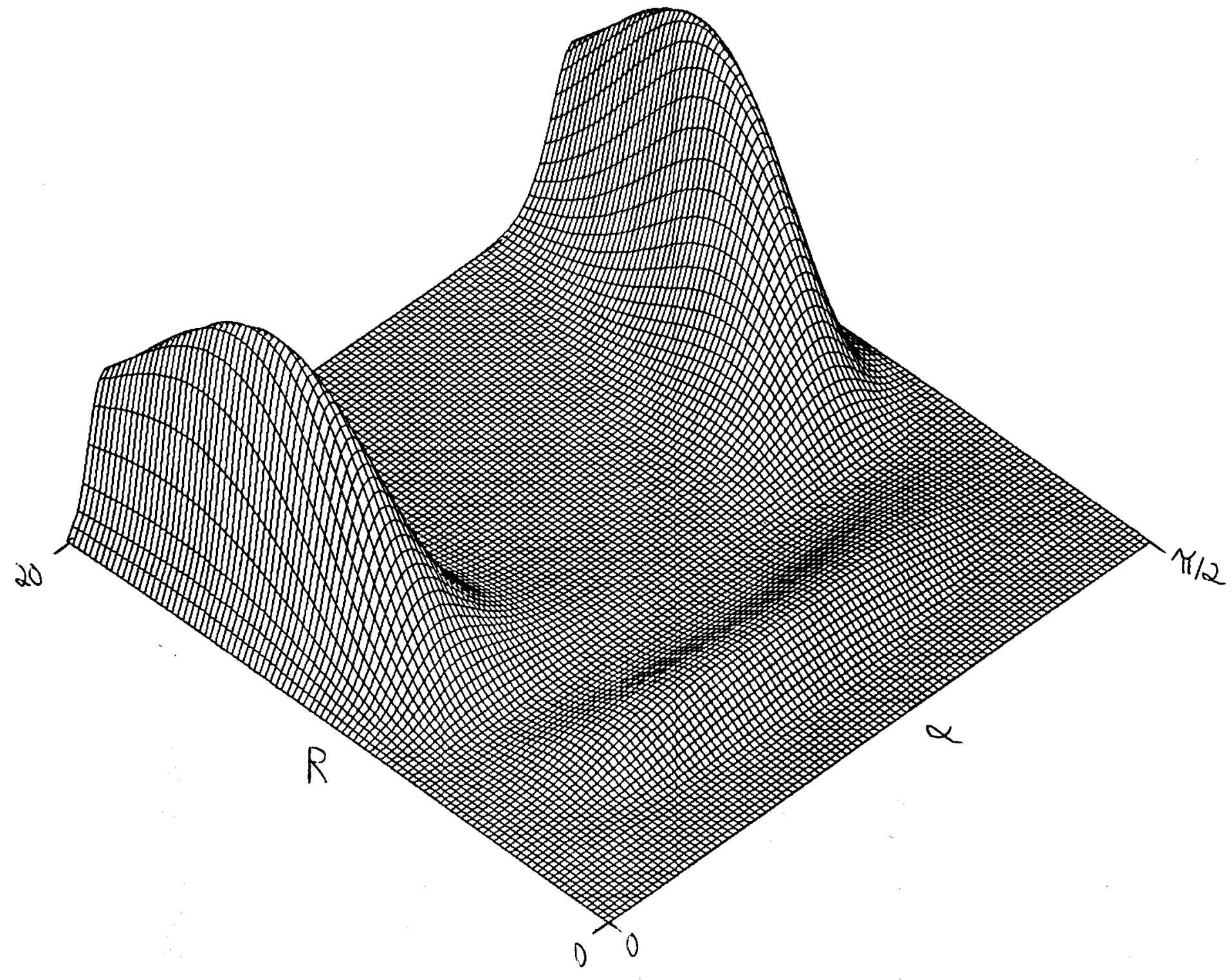

Figura 19: Densidade de probabilidade da função angular total correspondente a segunda energia da curva 2. 


\subsection{Aplicação da Diagonalização Laguerre-Jacobi}

\section{para o Átomo de Hélio}

Para obter os elementos de base (47), $\mathcal{G}_{\mathcal{\ell}_{\infty} \ell_{\epsilon}}$ é necessário pouco trabalho computacional. Este fato é devido a rápida convergência das soluções desacopladas da equação angular, com relação a expansão em séries de potencias na variável $z$. O método da Diagonalização Laguerre-Jacobi será aplicado ao átomo de hélio.

O sistema de coordenadas é o mesmo utilizado na seção anterior, assim como o processo de obtenção das funções de canal. A diferença dos procedimentos scrá o desacoplamento das equações angulares. Para testar o método, verifica-se quantos elementos da base são necessários para reproduzir os resultados da tabela 4. Na Tabela 5, estes elementos são representados por $N_{\max } \mathrm{e} \ell_{\max }$. Os índices correspondem ao valor máximo de $N_{\lambda} \mathrm{e}(\ell+1)$ utilizado para diagonalizar o hamitoniano angular.

\begin{tabular}{|c|c|c|c|c|c|}
\hline$\ell$ & 2 & 4 & 6 & 8 & 10 \\
\hline \multicolumn{6}{|l|}{$N_{\max }$} \\
\hline 0 & -8.481699458 & -8.490053951 & -8.490887318 & -8.491079545 & -8.491144130 \\
\hline 4 & -8.482303983 & -8.49093339900 & -8.191842842 & $-8.4920(33743$ & -8.492141406 \\
\hline 8 & -8.482314285 & -8.4909954834 & -8.491869982 & -8.492093202 & -8.492172742 \\
\hline 12 & -8.482315266 & -8.4909957190 & -8.491872499 & -8.492097187 & -8.492177194 \\
\hline 16 & -8.152315450 & $-8.4909956(882$ & -8.191873241 & -8.4920998131 & -8.492178292 \\
\hline Tabela 4 & -8.482315532 & -8.490957922 & -8.491873637 & -8.492098663 & -8.492178945 \\
\hline
\end{tabular}

Tabela 5: Análise de convergência da diagonalização Laguerre-Jacobi no ponto de mínimo de $U_{0} / R^{2}$ para - $H_{e}$ atômico. Os índices $N_{\max }$ e $\ell_{\max }$ correspondem ao valor máximo de $N_{\lambda}$ e $\ell+1$ que definem os elementos da base. Os valores de comparação são fornecidos na tabela 4.

Para a região assintótica de grandes valores de $R$, repete-se a análise anterior, verificandose uma convergência muito rápida na Tabela 6, como havia sido previsto. 


\begin{tabular}{||c|c|c|c|c|}
\hline$\ell$ & 2 & 3 & 4 & 5 \\
\hline$N_{\max }$ & & & & \\
\hline \hline 0 & -4.205786298 & -4.205786328 & -4.205786328 & -4.205786328 \\
\hline 4 & -4.205792993 & -4.205793167 & -4.205793171 & -4.205793171 \\
\hline 8 & -4.205795704 & -4.205795927 & -4.205795932 & -4.205795933 \\
\hline 12 & -4.205796146 & -4.205796377 & -4.205796382 & -4.205796382 \\
\hline 16 & -4.205796220 & -4.205796452 & -4.205796457 & -4.205796457 \\
\hline \hline exalo & -4.205796241 & -4.205796472 & -4.205796477 & -4.205796478 \\
\hline
\end{tabular}

Tabela 6: Mesma análise de convergência da tabela 5 , para a região assintótica com $R=10$.

\subsection{Excitons Ligados em Semicondutores}

Em 1964 Hopfield [23] utilizou o modelo da massa efetiva para analisar os sistemas excitônicos. Neste modelo um exciton é tratado como sendo formado por um par de partículas de cargas opostas interagindo via potencial de Coulomb. Às partículas, um elétron e um buraco, são atribuidas massas efetivas $m_{e}^{*}$ e $m_{b}^{*}$. Os efeitos do sistema de muitos corpos, que constitui o semicondutor, são incorporados neste modelo através do valor destas massas e da constante dielétrica de baixa frequência $\varepsilon$. A validade desta interpretação foi verificada experimentalmente, tornando possível o estudo de um problema complexo de estado sólido através de técnicas desenvolvidas para poucos corpos. Quando o exciton entra no campo de potencial de uma impureza de carga efetiva $e / \sqrt{\varepsilon}$ forma-se um sistema de três corpos caso a energia do conjunto seja menor que a energia da impureza neutralizada pelo elétron, caso contrário o buraco fica livre. Hopfield estimou 
as energias de ligação por extrapolaçao de cuergias moleculares. Cálculos mais acurados foram realizados por Rodriguez et al [24] e Suffczyuski et al [25,26] utilizando métodos variacionais.

A repulsão do buraco pela impurega gera cestados ligados de encrgia muito próxima do limite de ionização. Assim, a função de onda deste sistema deve representar simultaneamente uma partícula ligada e uma espalhada, dificultando a aplicação dos métodos variacionais. Este comportamento é tratado de modo natural com o método hiperesférico, sendo refletido na forma das curvas de potencial. Devido a grande quantidade de materiais semicondutores onde podem ser formados excitons ligados, existe a possibilidade de variar de modo quase continuo as características do sistema. Assim, estudo da transformação do problema de três corpos com a massa das partículas torna-se possivel tendo como base resultados experimentais.

Considerando a impureza com massa muito maior que o elétron e o buraco, faz-se $m_{1} \rightarrow \infty, m_{2}=m_{e}^{*}, m_{3}=m_{b}^{*}, q_{1}=Z e, q_{2}=-c$ e $q_{3}=e$. Define-se $m=m_{b}^{*} / m_{e}^{*}$. Com isso a interação $\hat{V}(\alpha, \theta)$ é dada por,

$$
\hat{V}(\alpha, \theta)=-\sqrt{\frac{m m_{e}^{*}}{\mu}} \frac{Z c^{2}}{\operatorname{senn} \alpha}+\sqrt{\frac{m_{b}^{*}}{\mu}} \frac{Z e^{2}}{\varepsilon \cos \alpha}-\sqrt{\frac{m_{e}^{*}}{\mu}} \frac{c^{2} / \varepsilon}{\sqrt{\operatorname{sen}^{2} \alpha+\frac{\cos ^{2} \alpha}{m}-\frac{\operatorname{sen}(2 \alpha) \cos \theta}{\sqrt{m}}}} .
$$

Na Figura 20 observa-se para o ZnSe a forma deste potencial com os pólos atrativos elétron-núcleo $\alpha=0$, elétron-buraco $(0, \alpha)=\left(0, \alpha_{-}\right)$e o pólo repulsivo $\alpha=\pi / 2$, onde $\operatorname{tg} \alpha_{-}=1 / \sqrt{m}$

Como foi citado, ao contrário do átomo da hílio, os estados ligados possucm energias muito próximas do limite de ionização. Por este motivo a formação de um estado ligado 
depende criticamente da correlação, e a convergência da curva de potencial com relação ao acoplamento de canais de momento angular é lenta. Isto pode ser observado na Figura 21 , onde mostra-se o comportamento da curva de potencial do estado fundamental com relação a $\ell_{\max }$ para um exciton ligado a uma impureza no semicondutor ZnSe. Uma análise mais detalhada pode ser vista na Tabela 7.

\begin{tabular}{||c|c|c|c||}
\hline $\operatorname{lmax} N$ & 80 & 100 & 120 \\
\hline \hline 3 & -18.2866453 & -18.2866453 & -18.2866453 \\
\hline 4 & -18.4908194 & -18.4908199 & -18.4908199 \\
\hline 5 & -18.6117268 & -18.6117254 & -18.6117257 \\
\hline 6 & -18.6861084 & -18.6860845 & -18.6860856 \\
\hline 7 & -18.7337109 & -18.7336006 & -18.7336026 \\
\hline 8 & -18.7653946 & -18.7650791 & -18.7650769 \\
\hline 9 & -18.7873147 & -18.7866360 & -18.7866133 \\
\hline
\end{tabular}

Tabela 7: Valores do ponto de mínimo de $U(R) / R^{2}$ (em meV) para a curva mais baixa de um exciton ligado a uma impureza de carga $e$ no semicondutor ZnSe.

Um efeito do truncamento no acoplamento angular antes de obter uma convergencia satisfatória, é a violaçào das inegualdades fornecillas na seçào 2.2 , onde assume-se a completeza da expansào da base angular. O fator $m$ define as duas regióes de expansão da função de onda angular $0 \leq \alpha \leq \alpha_{-}$e $\alpha_{-} \leq \alpha \leq \pi / 2$, caracterizando a natureza do sistema. A Figura 22 mostra as curvas de potencial de alguns materiais semicondutores nas unidades adimensionais, $h^{2}, e^{2} / \varepsilon, m_{e}^{*}=1$. A palavra "crit" refere-se a um material fictício para o qual nào há estado ligado. Já na Figura 23 observa-se outras curvas, onde nota-se que estes sistemas possuem energias de ligação muito pequenas e grandes dimensões. A Tabela 8 fornece os resultados destas energias na aproximação adiabática extrema para alguns materiais, em comparação com resultados variacionais. Foram acoplados oito canais 
de momento angular.

\begin{tabular}{||c||c|c|c|c|c|c||}
\hline material & $\varepsilon$ & $m_{e}^{*}(u m a)$ & $m_{b}^{*} / m_{e}^{*}$ & $E_{D}$ & $E-E_{D}(m e V)$ & $\left(E-E_{D}\right) / E_{D} \%$ \\
\hline \hline $\mathrm{lnSb}$ & 16.80 & 0.02 & 15.0 & $0.9(911$ & $0.089) 3,0.09993^{1)}$ & $9.2\left(6,10.30^{1)}\right.$ \\
\hline $\mathrm{ZnSe}$ & 9.10 & 0.10 & 6.00 & 16.43 & $0.751,0.676^{1)}$ & $4.57,4.12^{1)}$ \\
\hline $\mathrm{CdS}$ & 10.33 & 0.20 & 5.00 & 25.50 & $0.918,0.791^{2)}$ & $3.60,3.10^{2)}$ \\
\hline $\mathrm{ZnO}$ & 8.50 & 0.24 & 4.75 & 45.19 & $1.514,1.220^{2)}$ & $3.35,2.70^{2)}$ \\
\hline $\mathrm{CdSe}$ & 10.66 & 0.13 & 4.54 & 15.56 & $0.486,0.348^{1)}$ & $3.12,2.23^{1)}$ \\
\hline
\end{tabular}

Tabela 8: Energia de ligação para excitons ligados a impurezas de carga $e$ em vários materiais semicondutores. $E_{D}$ é a energia de dissociaçào. Os indices referem-se a cálculos variacionais: 1) referéncia 26 e 2) referência 27. As constantes dielétricas $\varepsilon$ são obtidas da referência 26.

A energia de ligaçào como funçào da massa $m$ é desenhada na Figura 24. As energias estão na forma de porcentagem da energia de ligaçào do sistema no limite de grandes valores de $R$, o que corresponde ao elétron ligado à impureza e o buraco, espalhado. Para grandes valores de $m$ o sistema aproxima-se da molécula $H_{2}^{+}$. Na região $2 \leq m \leq 6$, que inclui os semicondutores mais comuns, a energia de ligaçào mostra uma variação quase linear com relagào a $m$. Para valores menores que 2 , a energia, já muito pequena, tende a zero. Com isso, outras interaçoes negligenciadas nos cálculos podem perturbar o sistema e causar dissociação, impedindo a olsservação experimental da energia de ligação em sistemas com buracos de massa pequena com relação à do elétron.

Conforme o valor de $m$ diminui, o sistema solre uma transição da região tipo molecular para um setor deslocalizado, onde a funçào de onda é extendida para uma forma espalhada. Na Figura 25 este comportamento pode ser observado através da função de onda radial $\left|F_{0}(R)\right|^{2}$ para vários valores de $m$. Conforme $m$ diminui, o valor máximo da função de onda decresce e esta tende a dilatar para grandes valores do R. Outra forma de olservar 
este comportamento é dada pela Figura 26, na qual o valor médio do raio hiperesférico é plotado em função de $m$. Para $m$ grande $<R>$ tende lentamente a um valor constante. Para $m=5$ os sistemas Coulombianos atingem seu tamanho mínimo, e com valores menores o tamanho médio do sistema começa a divergir. Muitos valores da chamada

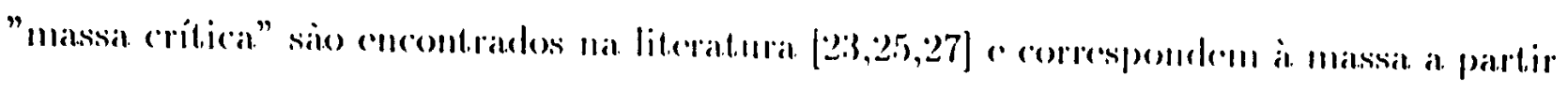
da qual existe um estado ligado de três corpos. $\Lambda$ parentemente este conceito está ligado à precisão com a qual os cálculos são realizados na região de massas pequenas.

Um fato observado na soluçào dos excitons ligados é a existencia de apenas um estado ligado. Isto ocorre devido à forma da curva de potencial de cada sistema. As curvas excitadas, ao contrário possuem em forma que sustenta um espectro tipo molecular, com energias separadas por intervalos constantes. Estas energias correspondem a estados ressonantes e são obtidas por meio da aproximaçào adliabálica. Caso o acoplamento radial fosse introduzido estes estados nào seriam observados. Na figura 27 é feita uma comparação da curva mais bajxa de um sistema de três corpos no semicondutor /ube a a primeira curva

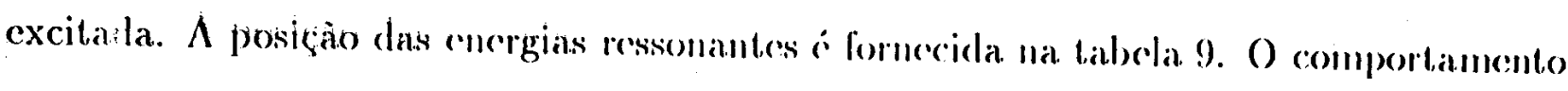
são de onda adiabática para alguns estados auto-ionizantes pode ser visto na figura 


\begin{tabular}{||c|c||}
\hline Estado & -Encrgia (moV) \\
\hline \hline 1 & 11.50 \\
\hline 2 & 10.10 \\
\hline 3 & 9.07 \\
\hline 4 & 8.31 \\
\hline 5 & 7.65 \\
\hline 6 & 6.96 \\
\hline 7 & 6.24 \\
\hline 8 & 5.52 \\
\hline 9 & 4.83 \\
\hline 10 & 4.19 \\
\hline
\end{tabular}

Tabela 9: Energias ressonantes de excitons ligatos a impurezas no Zubse correspondentes a primeira curva excitada 


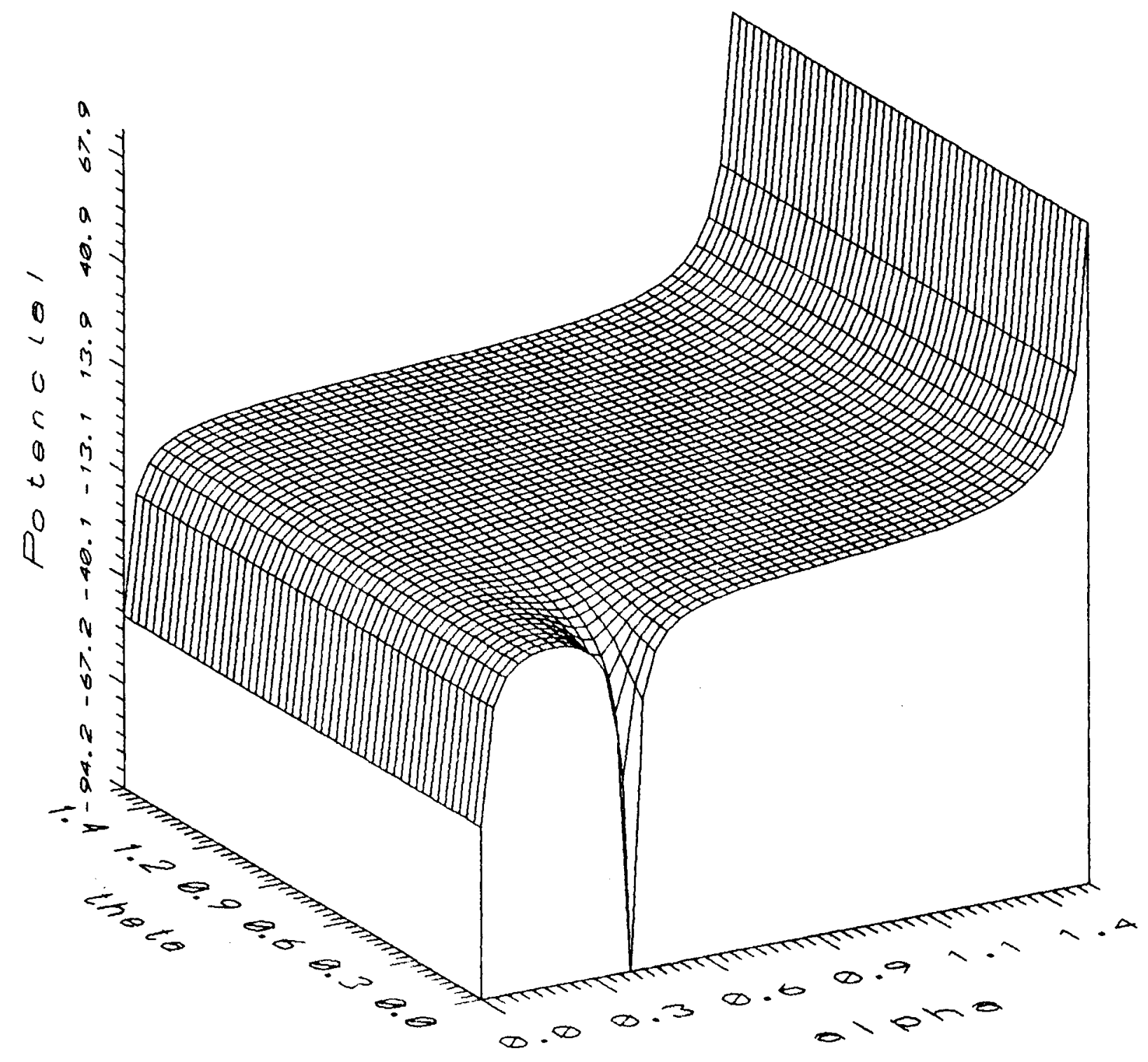

Figura 20: Potencial hiperesférico de um exciton ligado a uma impureza no semicondutor ZnSe. 


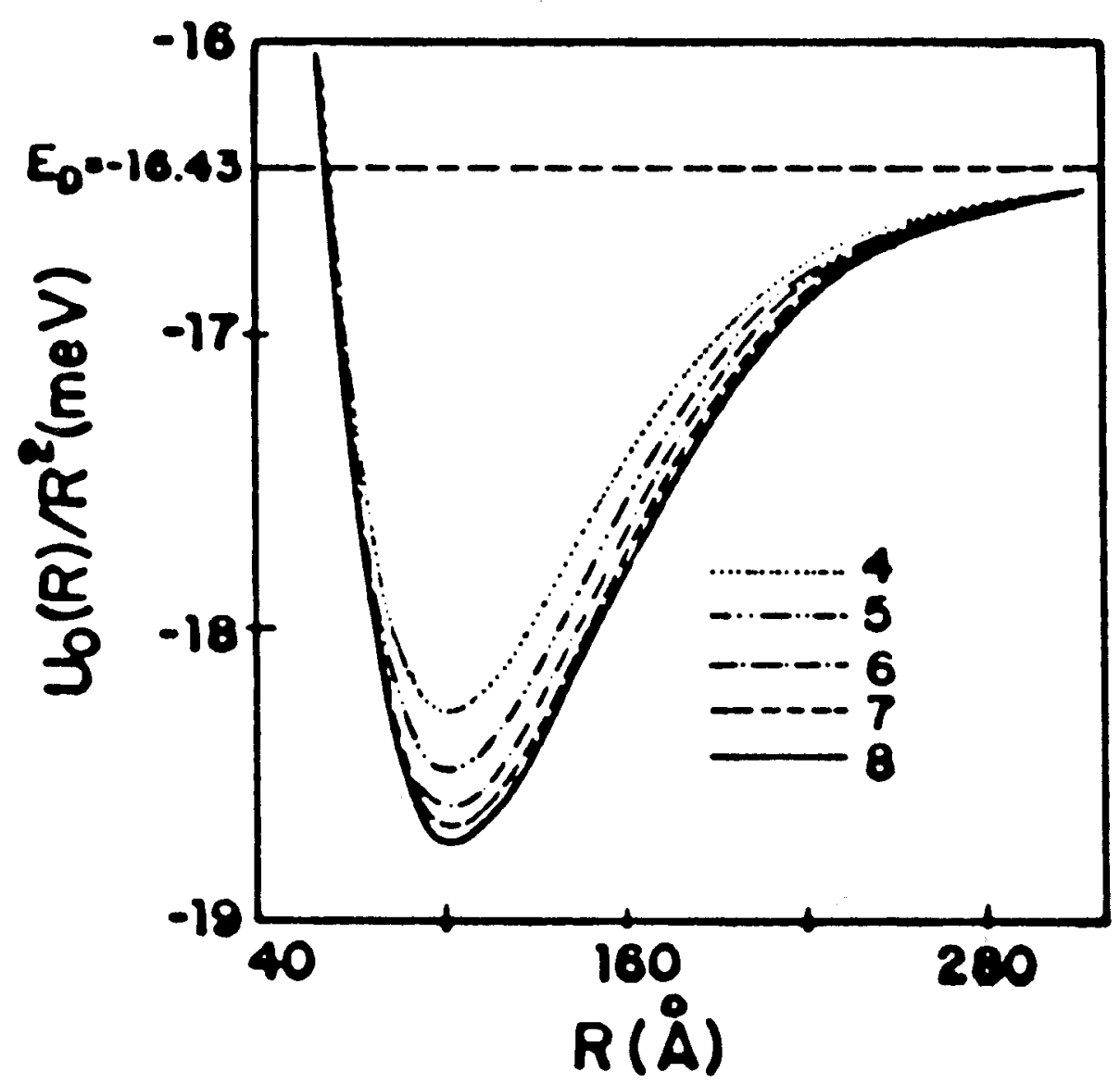

Figuta 21: Curvas de potencial como funço do numero de canais angulares para o ZnSe. 


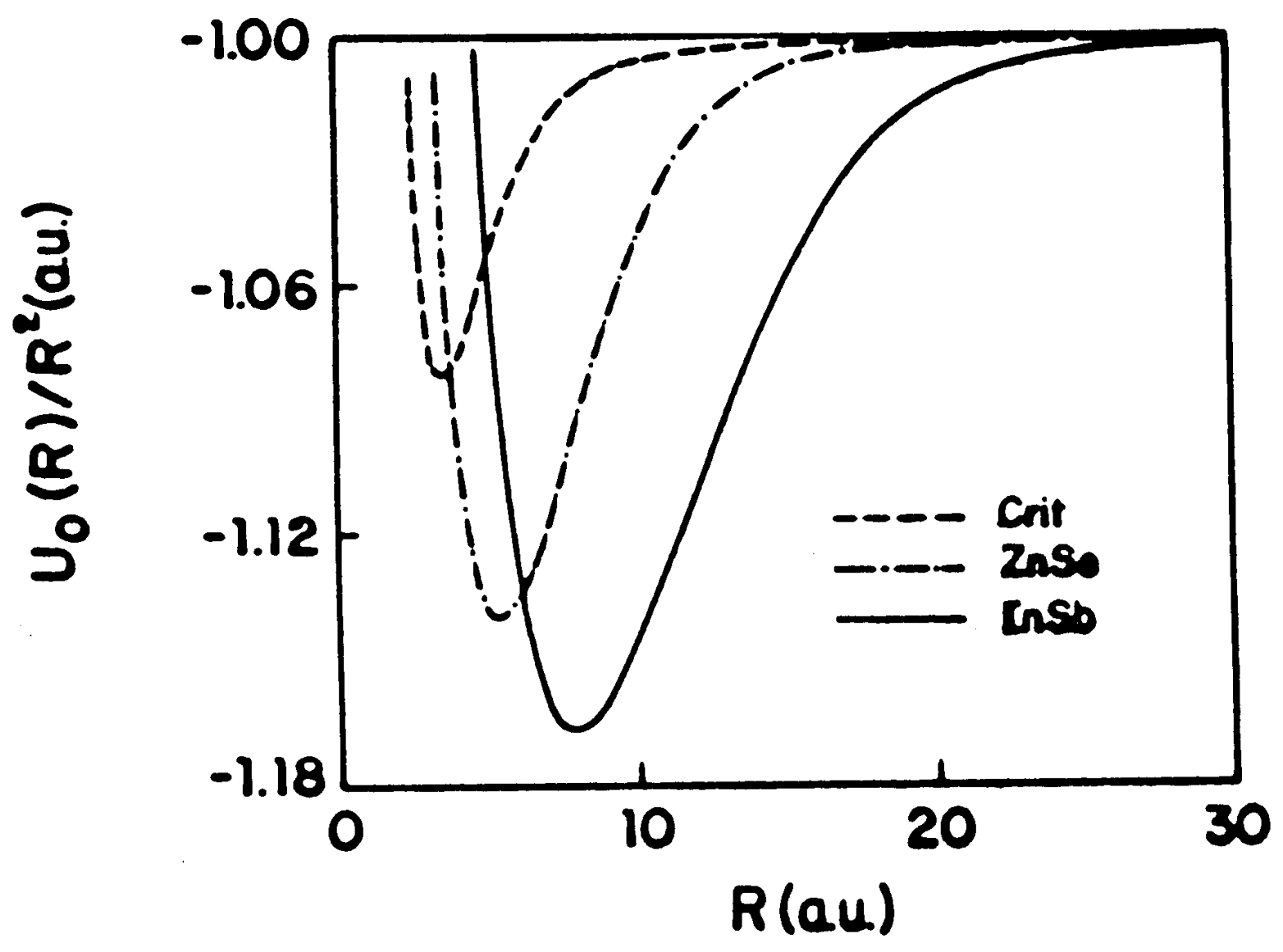

Figura 22: Curvas de potencial para diferentes materiais semicondutores em unidades adimensionais. 
Fighra 33: Curvás de potencial em meV para excitons ligados a impurezas. 


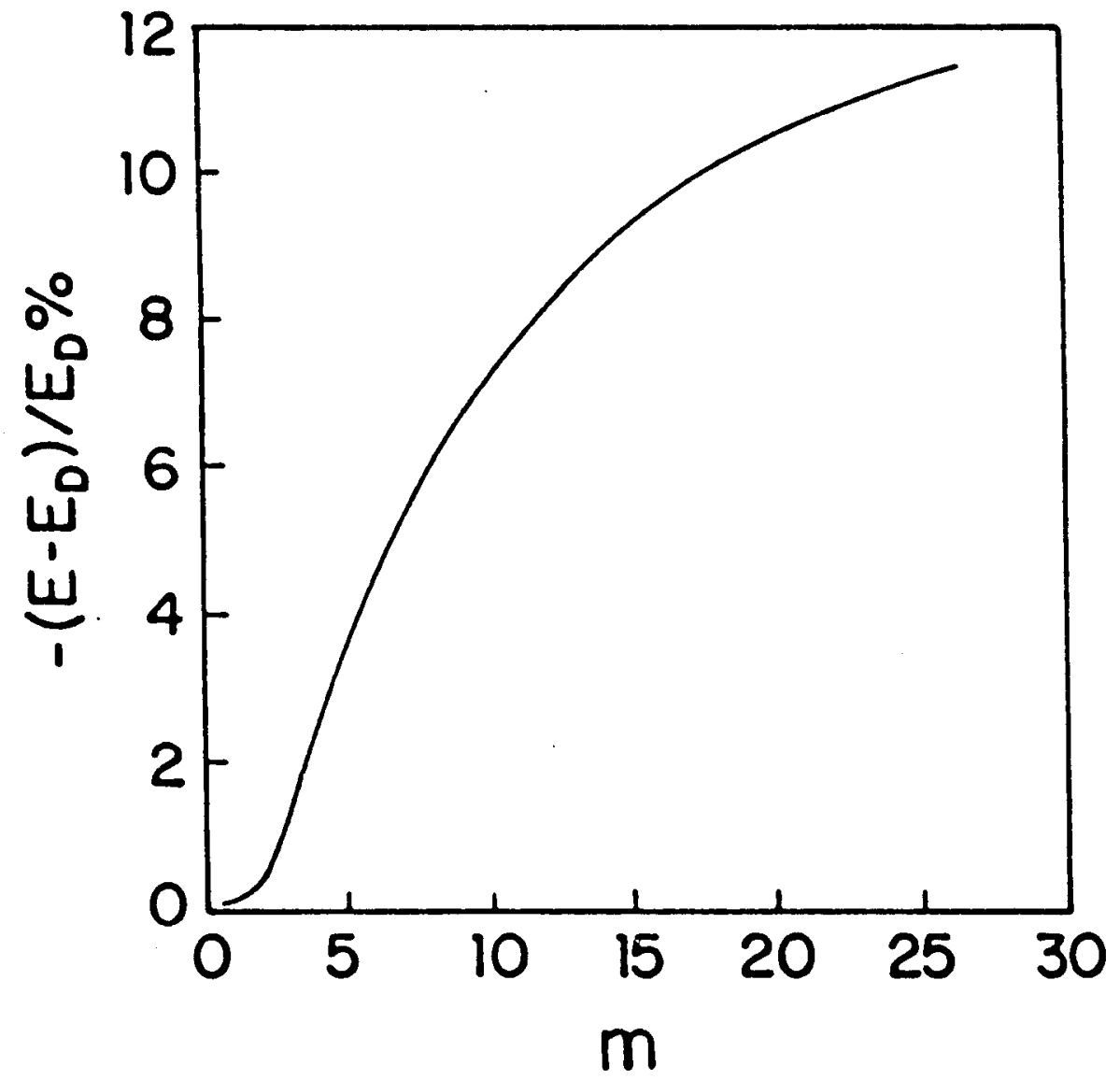

Figura 24: Comportamento da encrgia de ligaçào de excitons ligados com relaçào a razào de massa $m$ 


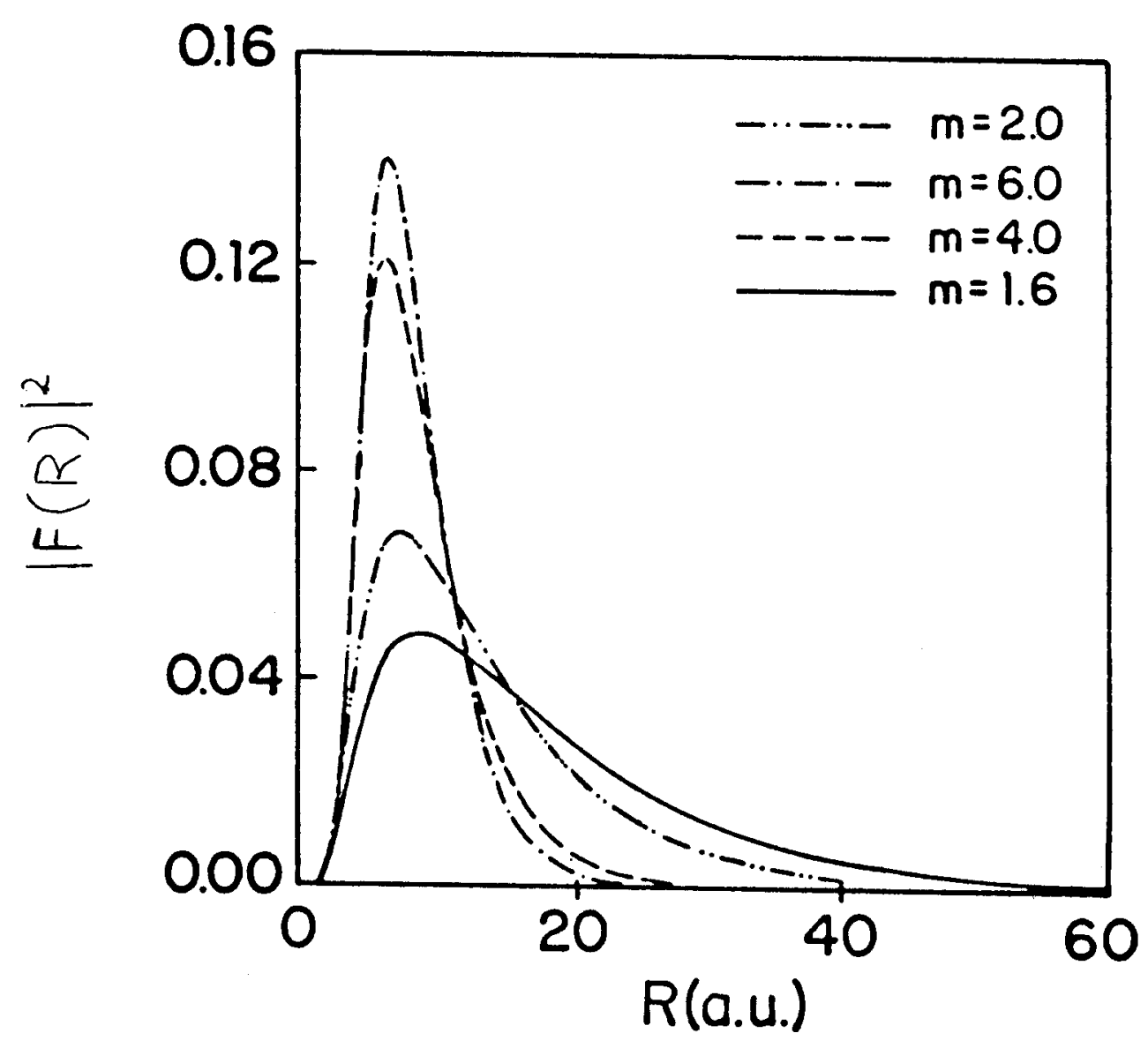

Figura 25: Densidade de probabilidade radial de excitons ligados em função de $m$. 


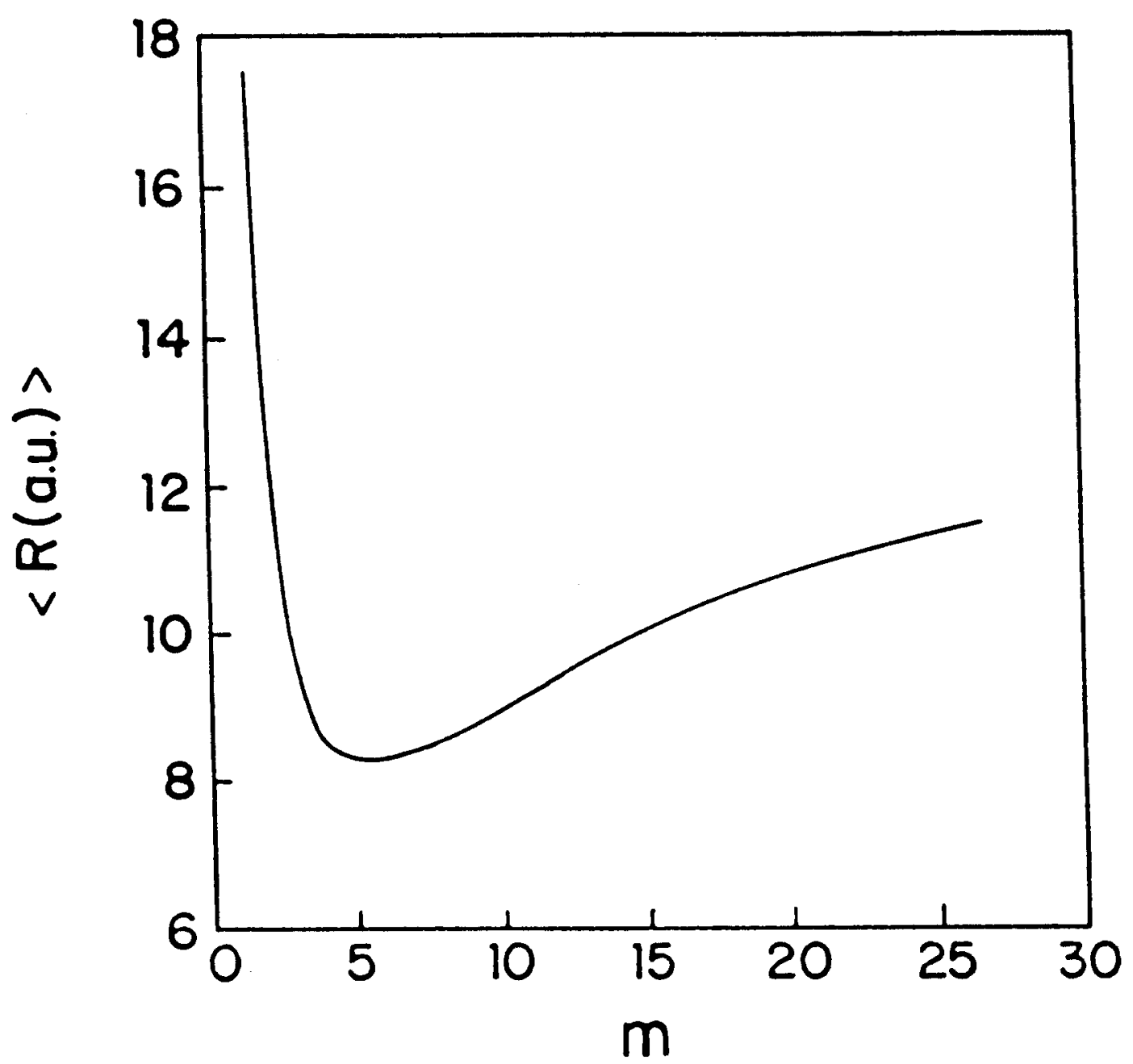

Figura 26: Comportamento do raio médio hiperesférico em função de $m$. 
Figura 27: Curvas de potencial mais baixas para excitons ligados em impurezas no semicondutor Zaste. 

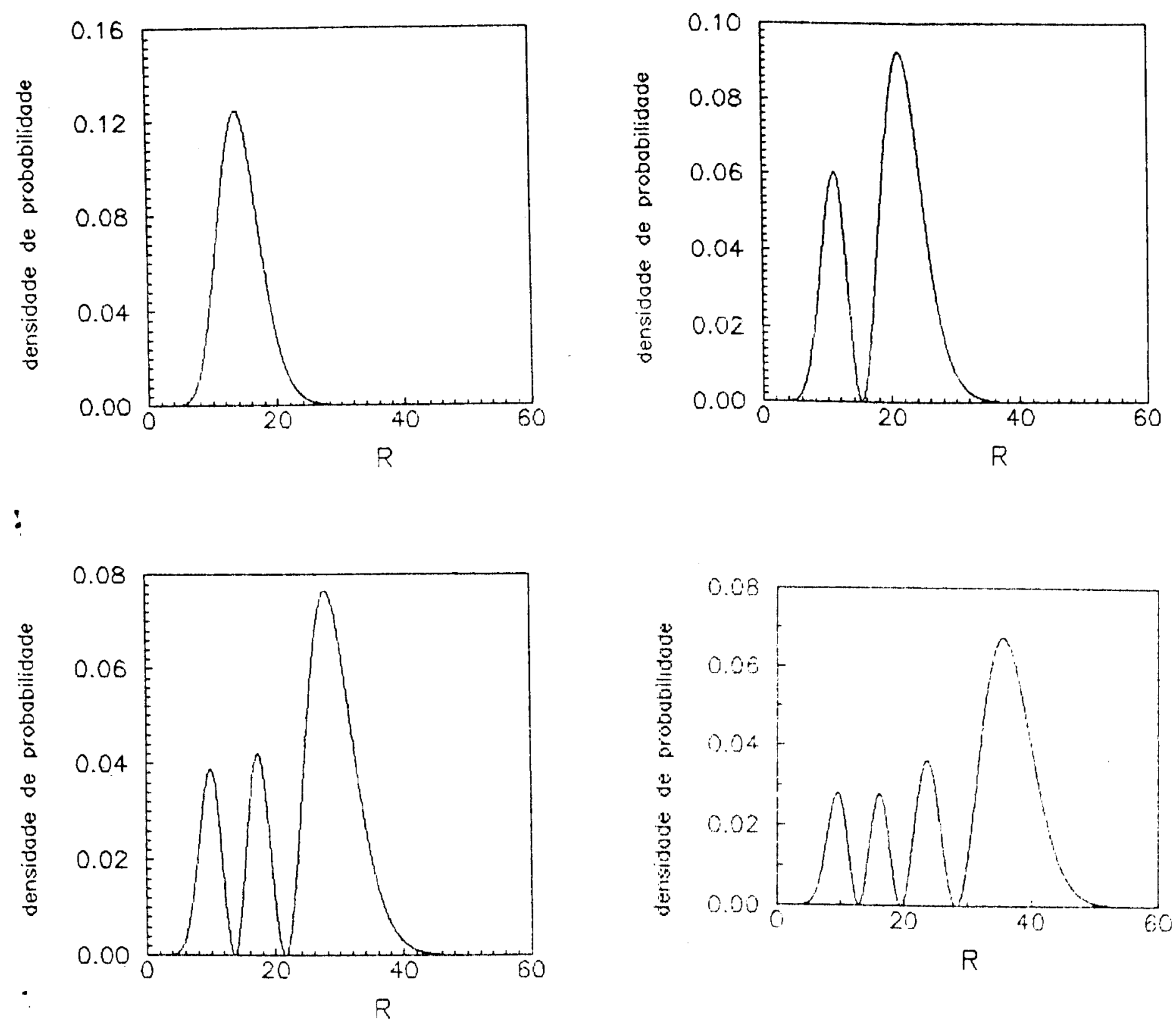

Figura 28: Densidade de probabilidade radial de estados excitados de excitons ligados a impurezas no senicondutor Znse. 


\section{4 Íon Negativo do Positrônio, $\mathrm{Ps}^{-}$}

O positrônio é um átomo formado por um elétron e sua anti-partícula, o pósitron, interagindo através do potencial de Coulomb. Fm 1946 J.A. Wheeler [28] demonstrou teoricamente a existência de um estado ligado do ion $P^{-} s^{-}$e, cm 1981, A.P. Mills [29] obteve a confirmação experimental. A produçào do $P s^{-}$t.en estimulado intensas investigações teóricas. Estuda-se a taxa de aniquilação elétron-pósitron, o comportamento dos estados auto - ionizantes c também busca-se informaçòes sobre a dinânica do problema de três corpos com relação a massa relativa das partículas. O problema encontrado no estudo deste sistema é a falta de um referêncial óbvio, como o cixo internulcar do $H_{2}^{+}$, ou então como no caso do $\mathrm{H}^{-}$, o potencial central de um próton fixo.

J. Rost e D. Wintgen [30] mostraram que o espectro ressonante do $P^{\prime} s^{-}$ć dominado pelo mesmo movimento quasi-separável do $\mathrm{H}_{2}^{+}$com o cixo "internuclear" elétron-elétron. Para provar este comportamento foram feitos 2 tratamentos para a equaçâo de Schrödinger não relativística. Utilizando coordenadas perimétricas obtiveram a posição das ressonâncias com precisào. Em seguida trataram o $\mathrm{PS}^{-}$com o método molecular adiabático (MA), derivado do Born-Oppenheimer. O resultado observado mostrou grande correspondência entre os métodos, levando à conclusão de que este sistema pode ser classificado com números quânticos de moléculas diatômicas. A aplicação do MA mostrou limitações no cálculo de estados muito excitados, onde é esperada uma falha da aproximação adiabática, mas também falhou para o estado fundamental c primeiras ressonâncias. A explicação está no fato das massas das três partículas serem da mesma grandeza, de modo que 
para as energias mais baixas, onde o tamanho do sistema é menor, a correlação entre as partículas impede a escolha de um eixo preferencial. Assim o eixo elétron-elétron deixa de ser uma boa coordenada adiabática. Por outro lado, a coordenada hiperesférica radial $R$ está relacionada com o tamanho do sistema, o que deve permitir o estudo adiabático do $\mathrm{Ps}^{-}$, mesmo quando considera-se os estados mais baixos.

Para tratar o $\mathrm{Ps}^{-}$com o método hiperesférico escolhe-se as coordenadas dos elétrons como $\vec{r}_{1}$ e $\vec{r}_{2}$ e do pósitron $\vec{r}_{3}$. As massa são $m_{e}$ e as cargas possuem o mesmo módulo $e$. O potencial hiperesférico

$$
\begin{array}{r}
\hat{V}(\alpha, \theta)=-\frac{e^{2}}{\operatorname{sen} \alpha}-\frac{\sqrt{2} e^{2}}{\sqrt{\operatorname{sen}^{2} \alpha+3 \cos ^{2} \alpha-\sqrt{3} \operatorname{sen}(2 \alpha) \cos \theta}} \\
+\frac{\sqrt{2} e^{2}}{\sqrt{\operatorname{sen}^{2} \alpha+3 \cos ^{2} \alpha+\sqrt{3} \operatorname{sen}(2 \alpha) \cos \theta}} .
\end{array}
$$

apresenta pólos para $\alpha=0,(\theta, \alpha)=\left(0, \alpha_{-}\right)$e $\left(\pi, \alpha_{+}\right)$onde $\operatorname{tg} \alpha_{ \pm}=\sqrt{3}$. Na figura 29 está representada a forma deste potencial em unidades adimensionais. O método MA e o hiperesférico fornecem a energia adiabática através de equaçòes radiais muito semelhantes. A principal diferença está no cálculo das equaçòes angulares. O primeiro resolve equações separáveis em coordenadas esferoidais confocais, encquanto que no método $\mathrm{H} A$ as equações são acopladas. Utilizando a aproximaçào adiabática extrema obteve-se a partir da curva de potencial mais baixa (Fig. 29) a energia do estado fundamental. O valor calculado foi de $E=-0.26467(a . u$.$) , enquanto que o resultado mais preciso na literatura é de$ $E=-0.262005$ (a.u.) obtido por Petelenz [31] através do método variacional. O erro de $1 \%$ mostra que à coordenada $R$ é uma varíavel adial)ática adequada a cste tipo de sistema. 


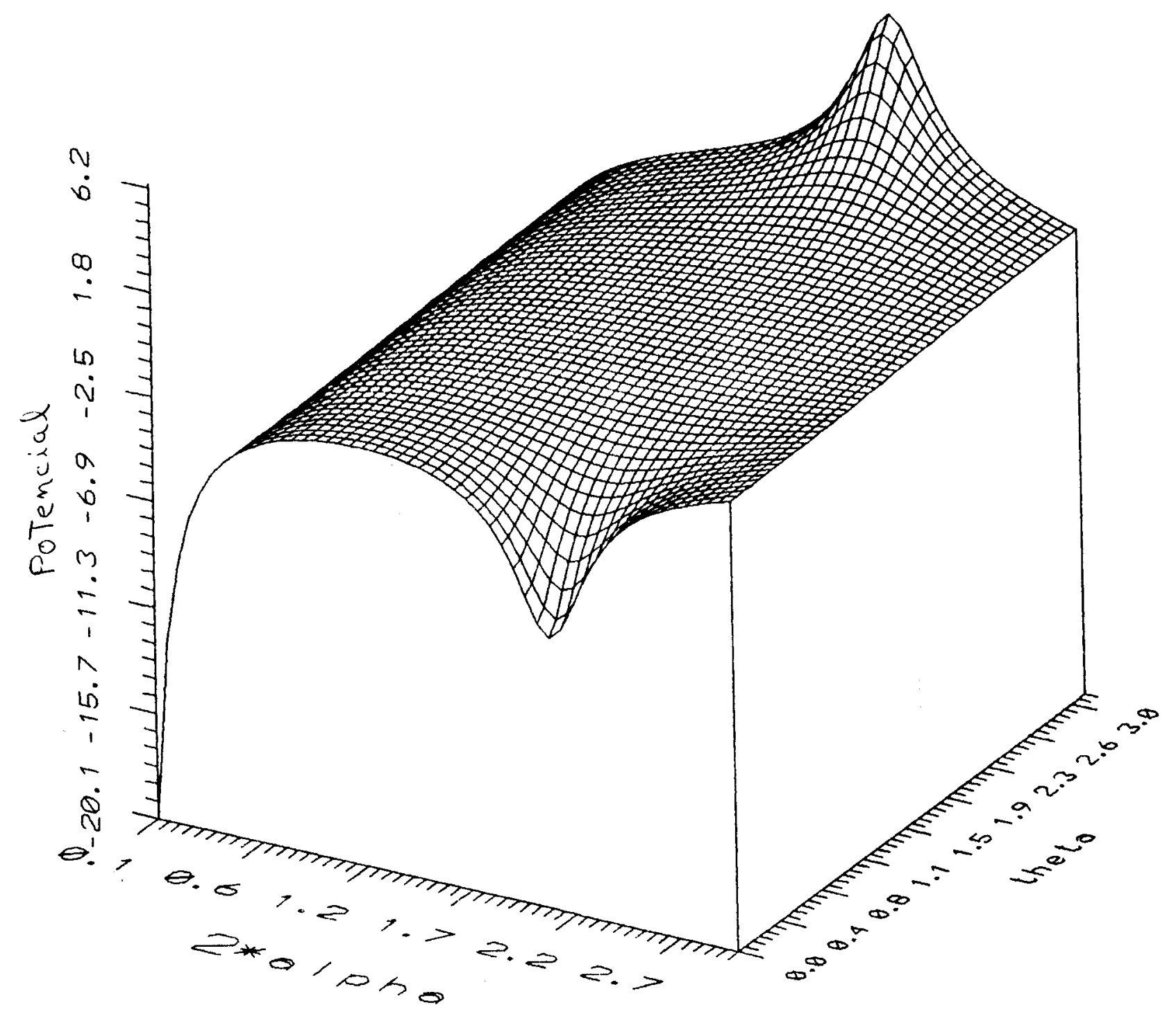

Figura 29: Potencial hiperesférico para o $P^{3} s^{-}$. 


\section{$4.5 d d \mu$ e $d_{2}^{+}$}

Um dos interesses no estudo do $d d \mu$ está na comprecnsão do mecanismo de fusão catalizada por Muon. Esta reaçào, prevista por Frank [32] em 1947 e Salikarov [33] em 1948, ocorre porque a tempo de formaçào do estado ligado de três corpos, seguido da reação, é menor que o de decaimento do muon. A verificaçà experimental foi feita em 1957 por Alvarez [34]. Os núcleos reagem quando estào ao alcance das forças nucleares, necessitando para isso vencer a barreira de potencial Coulombiana. A probabilidade com que os núcleos tunelan esta barrcira ć obtida através do cálculo da funçăo de onda do sistema Coulombiano de três corpos. Para o de esta probabilidade ć insignificante ( $10^{-54}$ reaçòes/segundo), mas trocando o elétron pelo muon, o tamanho médio do sistema fica cerca de duzentas vezes menor, e a probabilidade de reação atinge o valor de $10^{11}$ reações por segundo. Este processo nào pode ser aplicado como fonte de energia porque a fusão gera um núcleo de hélio que captura o muon, impedindo novas catálises. Ultimamente vem sendo estudados processos de fusào a partir de estados ressonantes, para os quais o tamanho médio do sistema a major que dos cstandes ligados, diminumelo a possibilidade de que o muon venha a ser apturado. Alóm do cstudo do estado ligado deste problema, a aplicação do método hiperesférico visa testar as técuicas desenvolvidas nas seçóes anteriores, uma vêz que os sistemas moleculares dificultann a convergência da soluçăo mumérica da. função de onda angular.

As coordenadas $\vec{r}_{1}$ e $\vec{r}_{2}$ sào escolhidas para os deutérios e $\vec{r}_{3}$ para o muon. O potcncial hiperesférico para este sistema de coordenadas appresenta uma forte repulsão em $\alpha=0$ e 
pólos atrativos simétricos com relação ao ângulo 0. Sua forma (Fig.30) é a seguinte,

$$
\begin{array}{r}
\hat{V}(\alpha, \theta)=\frac{e^{2}}{\operatorname{sen} \alpha}-\frac{\sqrt{2} e^{2}}{\sqrt{\operatorname{sen}^{2} \alpha+\eta_{-}^{2} \cos ^{2} \alpha-\eta_{-} \operatorname{sen}(2 \alpha) \cos \theta}} \\
-\frac{\sqrt{2} c^{2}}{\sqrt{\operatorname{sen}^{2} \alpha+\eta_{+}^{2} \cos ^{2} \alpha+\eta_{+} \operatorname{sen}(2 \alpha) \cos \theta}}
\end{array}
$$

onde $\eta_{+}^{2}=\eta_{-}^{2}=\left(2 m_{1} / m_{3}+1\right)$. Esta escolha de coordenadas faz com que no limite de $R$ grande a curva de potencial va a zero, ao contrário dos sistcmas já descritos, para os quais neste limite as curvas tendem ao potencial de dissociação. Sua convergência é mostrada na Figura 31, onde observa-se a forte dependencia do comportiuncuto assintótico com relação ao número de equações acopladas. Na tabela 10 sào fornecidos os valores de $U_{\lambda}(R) / R^{2}$ da curva mais baixa no seu ponto de mínimo, como função de $N$ e $\ell_{\max }$.

\begin{tabular}{||c|c|c|c|c||}
\hline $\operatorname{lmax} N$ & 150 & 250 & 350 & 450 \\
\hline \hline 1 & 2816.1183 & 2816.1183 & 2816.1183 & 2816.1183 \\
\hline 2 & 3154.3412 & 3154.3408 & 3154.3408 & 3154.3408 \\
\hline 3 & 3219.4164 & 3219.3978 & 3219.3978 & 3219.3978 \\
\hline 4 & 3237.5066 & 3237.41339 & 32237.4138 & 3237.4138 \\
\hline 5 & 3244.2197 & 3244.0502 & 3244.0496 & 3244.0494 \\
\hline
\end{tabular}

Tabela 10: Análise de convergencia da curva de potencial $-l l(R)_{0} / R^{2}(\mathrm{em} \mathrm{eV})$ do ddp para seu ponto de minimo.

Como exemplo final, na Figura 32 está a curva cle potencial mais baixa com três canais de momento angular acoplados para o $d_{2}^{+}$. Sua singularidade é muito próxima de $z=1$ (Tab.1), causando uma convergência lenta na expanısào em séries de polencias da função angular. 


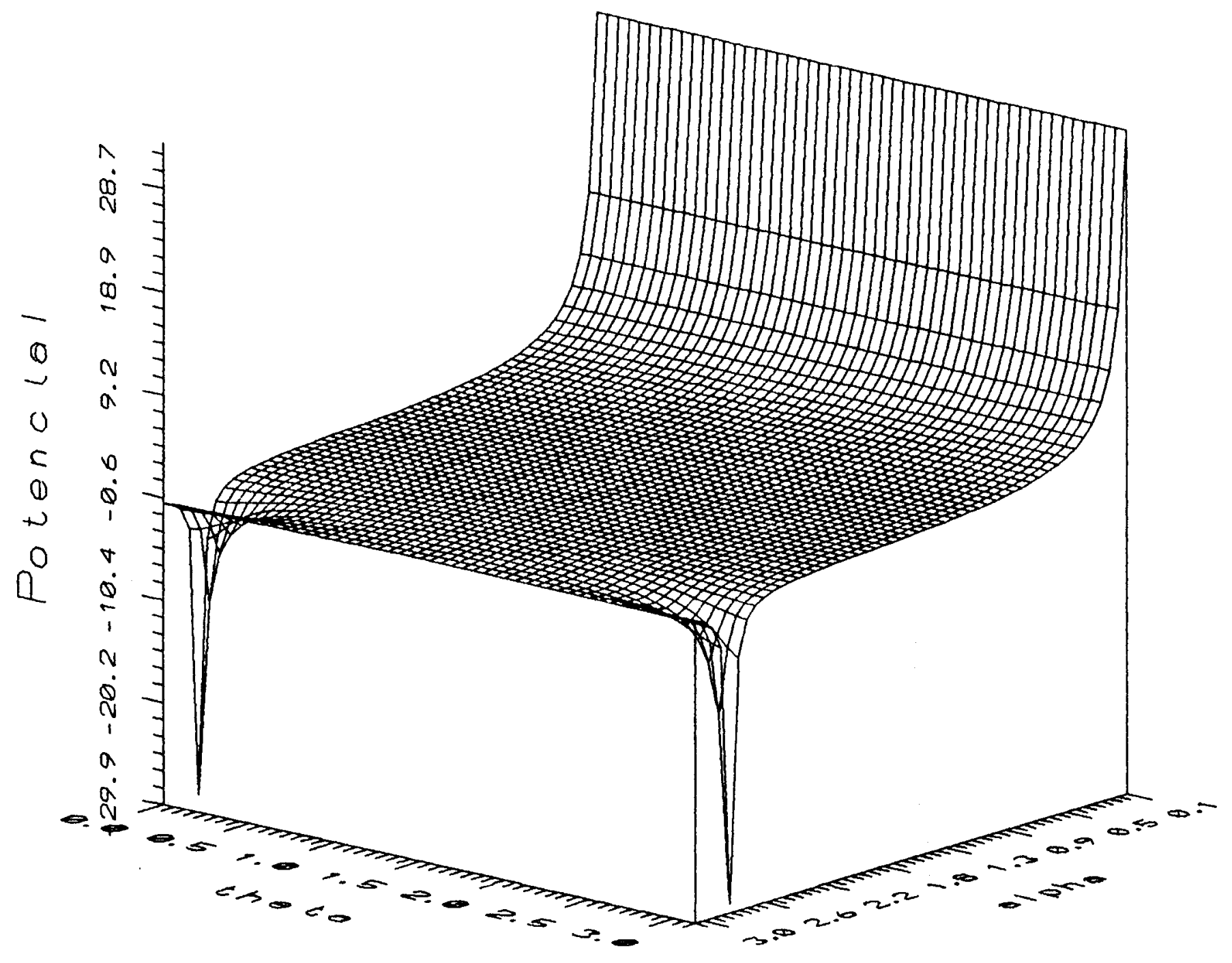

Figura 30: Potencial hiperesférico para o sistema $d d \mu$. 


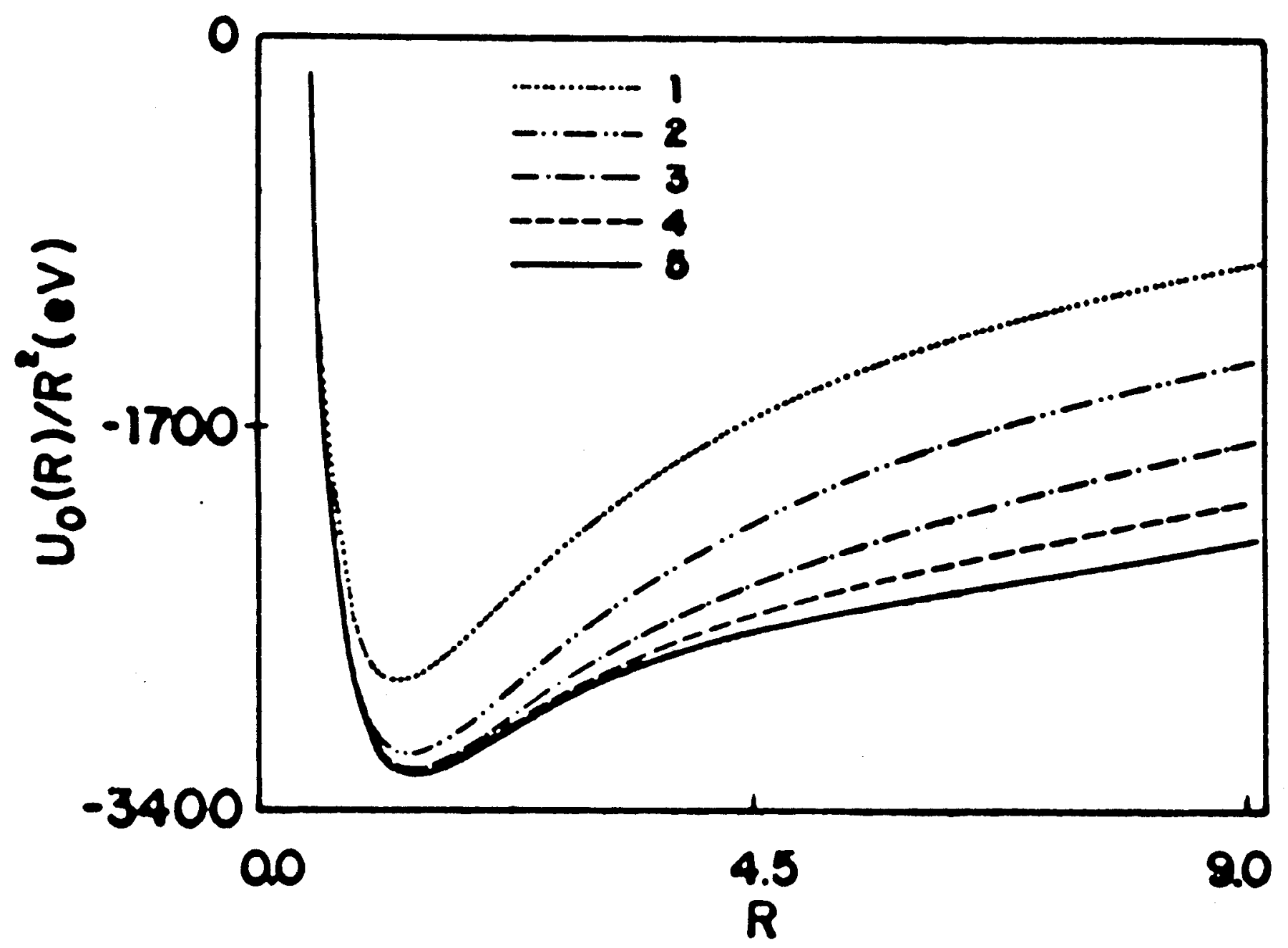

Figura 31: Curvas de potencial en funçào do acoplamento de cantais angulares para o sistema $d d \mu$. 


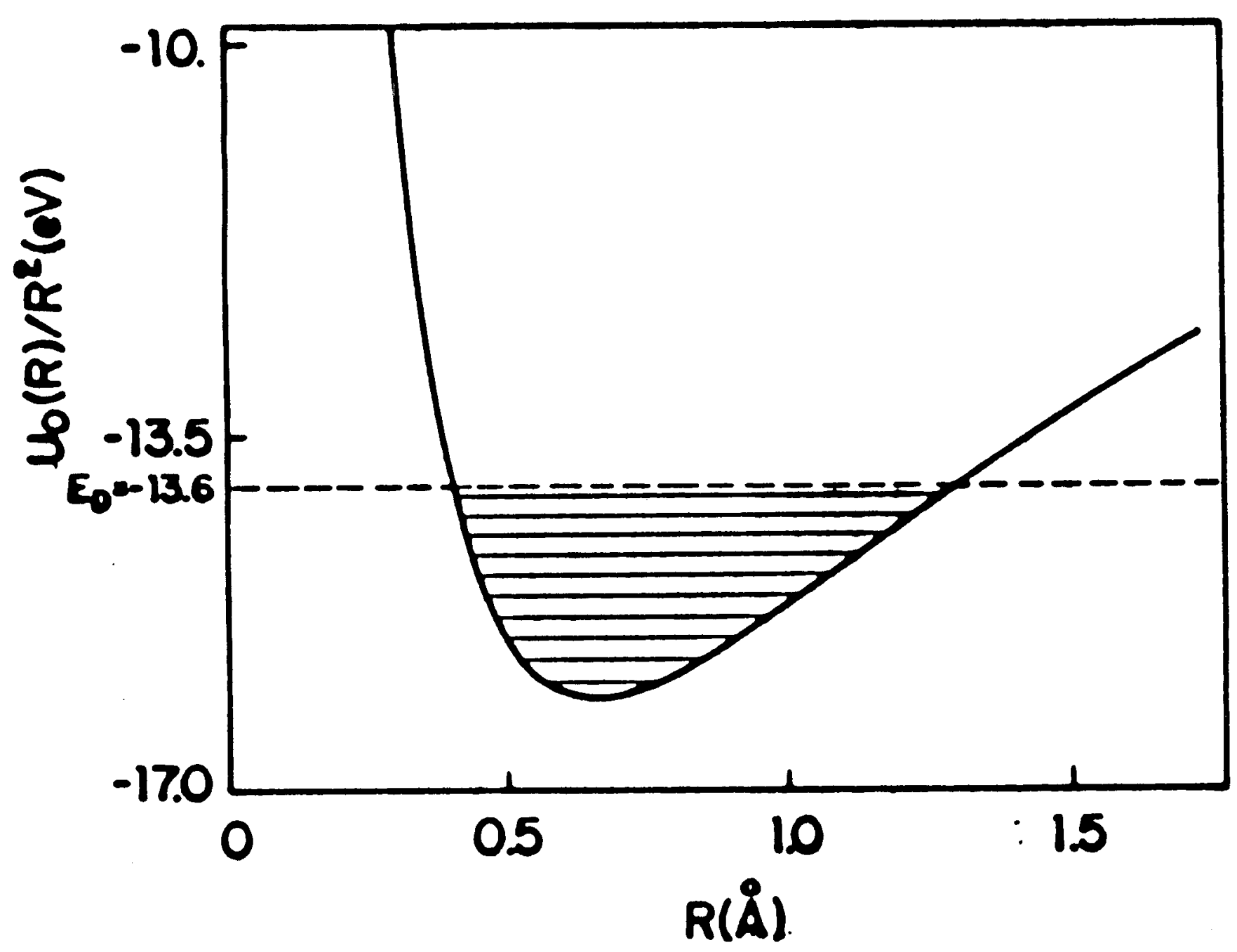

Figura 32: Curva de potencial mais baixa do $d_{2}^{+}$com energias olttidas na aproximação adialsática extrema. 
Capítulo 5

Conclusão 
Nesse trabalho foi desenvolvido um novo método que permite o cálculo de curvas de potencial para o problema Coulombiano de três corpos $\mathrm{cm}$ coordenadas hiperesféricas. Ele generaliza as técnicas baseadas na obtenção de uma solução analítica expandida em séries de potencia utilizada com sucesso na análise de átomos de dois elétrons. Inicialmente foran incorporados sistemas de massas variaiveis que exigem uma redefinição das condiçôes de contorno para o problema. Aplicaçôes ao problema de excitons aprisionados em impurezas em semicondutores decorreram desta generalização. Num segundo passo foram incorporados os efeitos de centro de massa, necessários para a análise de sistemas moleculares. Curvas de potencial para íons moleculares como $d_{2}^{+}, d d \mu$, e $P_{\mathcal{S}^{-}}$puderam ser calculadas.

Graças a eficiência das técnicas desenvolvidas foi possível uma reanálise das aplicações do método hiperesférico ao átomo de hélio, proporcionando um valor $-2.90372 a . u$. para a energia do estado fundamental, próximo do estado de arte, com apenas três curvas de potencial. Finalmente, uma técnica alternativa baseada na construção das funçẽs de Laguerre-Jacobi foi desenvolvida visando a aplicasào ao problema de muitos corpos.

A aplicaçào ao problena de excitons en semicondutores mostrou que a existência de una massa crítica para a qual nào existo estado ligado nesses sistemas é fruto de limitaçöes computacionais. Os resultados indican que na medida em que a precisão é aumentada, novos valores para a massa crítica sào obtidas

A eficiência do procedimento desenvolvido neste trabalho permite a curto prazo sua aplicaçào a vários problemas. Podem ser obtidas soluçôes não adiabáticas, estados resso- 
nantes de todos os sistemas descritos neste trabalho, além de outros como $d t_{\mu}, p d \mu, e c / \mu$ e sistemas confinados em pontos quânticos em semicondutores. Além disso, efeitos de centro de massa em excitons ligados, e o estudo da probabilidade de superposiçào das partículas para reagòes de aniquilancento on fusào, tambóm podem ser estudados. As técnicas desenvolvidas aqui serào futuramente generalizadas para problemas de mais corpos. 


\section{APÊNDICE A}

Cálculo dos Corficientess de Acoplamento Angular 
Para calcular os elementos de matriz de $v$ na equação (32), desenvolve-se o tensor

$$
\mathcal{T}_{\ell_{1} \ell_{2} \ell_{1}^{\prime} \ell_{2}^{\prime}}^{L M}(\alpha)=\int d \hat{\xi}_{1} d \hat{\xi}_{2} \frac{\mathcal{Y}_{\ell_{1}^{\prime} \ell_{2}^{\prime}}^{L M}\left(\hat{\xi}_{1}, \hat{\xi}_{2}\right)^{*} \mathcal{Y}_{\ell_{1} \ell_{2}}^{L M}\left(\hat{\xi}_{1}, \hat{\xi}_{2}\right)}{\sqrt{\operatorname{sen}^{2} \alpha+\eta_{ \pm}^{2} \cos ^{2} \alpha \pm \eta_{ \pm} \operatorname{sen} 2 \alpha \cos \theta}}
$$

O termo de raiz quadrada pode ser escrito na forma,

$$
f\left(1+t^{2} \pm 2 t x\right)^{-1 / 2}
$$

onde as expressòes para $f$ e $t$ sào fornecidas na tabola 11 .

\begin{tabular}{|c|c|c|c|c|}
\hline intervalo $\alpha$ & $f$ & $t$ & $f$ & $t$ \\
\hline \hline $0 \leq \alpha \leq \alpha_{-}$ & $\left(\eta_{+} \cos \alpha\right)^{-1}$ & $\operatorname{tg} \alpha / \eta_{+}$ & $\left(\eta_{-} \cos \alpha\right)^{-1}$ & $\operatorname{tg} \alpha / \eta_{-}$ \\
\hline$\alpha_{-} \leq \alpha \leq \alpha_{+}$ & $\left(\eta_{+} \cos \alpha\right)^{-1}$ & $\operatorname{tg} \alpha / \eta_{+}$ & $(\operatorname{sen} \alpha)^{-1}$ & $\eta_{-} \operatorname{colg} \alpha$ \\
\hline$\alpha_{+} \leq \alpha \leq \pi / 2$ & $(\operatorname{sen} \alpha)^{-1}$ & $\eta_{-} \operatorname{colg} \alpha$ & $(\operatorname{sen} \alpha)^{-1}$ & $\eta_{-} \operatorname{colg} \alpha$ \\
\hline
\end{tabular}

Tabela 11: Expressoes para te f da cequaçio (69).

Sabe-se pela definiçào dos polinômios de Legendre [21] que,

$$
\left(1+\ell^{2} \pm 2 l x\right)^{-1 / 2}=\sum_{L^{\prime}=0}^{\infty}(\mp)^{l^{\prime}} \iota^{l^{\prime}} P_{L^{\prime}}(x), \quad|\ell| \leq 1
$$

Usando o teorema da adigào para $P_{L}(x)$ e o falo que

$$
\mathcal{Y}_{\ell_{1} \ell_{2}}^{L M}\left(\hat{\xi}_{1}, \hat{\xi}_{2}\right)=\sum_{m_{1}, m_{2}}<L M \mid \ell_{1} m_{1} \ell_{2} m m_{2}>Y_{\ell_{1} m_{1}}\left(\hat{\xi}_{1}\right) Y_{\ell_{2} m_{2}}\left(\hat{\xi}_{2}\right)
$$

são obtidas as expressões

$$
<\hat{v}>=\sqrt{\frac{\mu_{1}}{\mu}} \frac{q_{1} q_{2}}{\operatorname{sen} \alpha} \delta_{\ell_{1} \ell_{1}^{\prime} \ell_{2} \ell_{2}^{\prime}}+\frac{m_{1}}{\sqrt{\mu \mu_{1}}} q_{1} q_{3} \Delta_{\ell_{1} \ell_{1}^{\prime} r_{2} r_{2}^{\prime}}^{+}(\alpha)+\frac{m_{2}}{\sqrt{\mu \mu_{1}}} q_{2} q_{3} \triangle_{\ell_{1} \ell_{1}^{\prime} \ell_{2} \ell_{2}^{\prime}}^{-}(\alpha)
$$

onde

$$
\triangle_{\ell_{1} \ell_{1}^{\prime} \ell_{2} \ell_{2}^{\prime}}^{ \pm}(\alpha)=\sum_{L^{\prime}=0}^{\infty}(\mp)^{L^{\prime}} \mathcal{C}_{\ell_{1} \ell_{1}^{\prime} \ell_{2} \ell_{2}^{\prime}}^{L L_{2}^{\prime}} \mathcal{D}_{L^{\prime}}^{ \pm}(\alpha)
$$




$$
\begin{array}{r}
\mathcal{C}_{\ell_{1} \ell_{1}^{\prime} \ell_{2} \ell_{2}^{\prime}}^{L L^{\prime}}=(-)^{L+L^{\prime}}\left[\left(2 \ell_{1}+1\right)\left(2 \ell_{1}^{\prime}+1\right)\left(2 \ell_{2}+1\right)\left(2 \ell_{2}^{\prime}+1\right)\right]^{1 / 2}\left[\begin{array}{ccc}
\ell_{1} & \ell_{1}^{\prime \prime} & \mathrm{L}_{1}^{\prime} \\
0 & 0 & 0
\end{array}\right] \\
{\left[\begin{array}{ccc}
\ell_{2}^{\prime} & \ell_{2}^{\prime} & \mathrm{I}^{\prime} \\
0 & 0 & 0
\end{array}\right]\left(\begin{array}{ccc}
\ell_{1}^{\prime} & \ell_{2} & \mathrm{~L} \\
\ell_{1}^{\prime} & \ell_{2}^{\prime} & \mathrm{L}^{\prime}
\end{array}\right)}
\end{array}
$$

onde aparecem os símbolos 3- $j$ e 6- $j$. Os termos de $\mathcal{D}_{l,}^{ \pm}$sào dados por

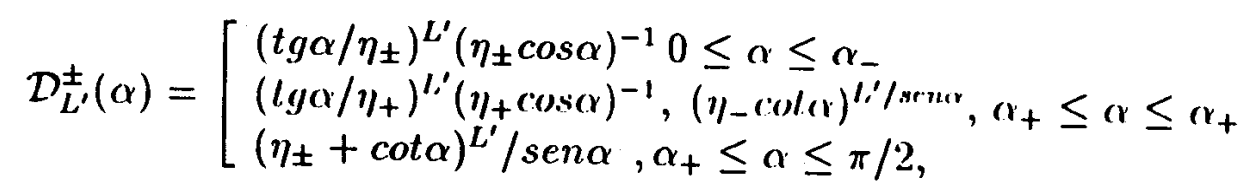

$\operatorname{com} \operatorname{tg} \alpha_{ \pm}=\eta_{ \pm}$. Em termos da variável $z, \mathcal{D}_{L^{\prime}}^{ \pm}(z)$ pode ser obtido notando que tgo $=$ $2 z /\left(1-z^{2}\right), \cot \alpha=\left(1-z^{2}\right) / 2 z, 1 / \operatorname{sen} \alpha=\left(1+z^{2}\right) / 2 z \mathrm{e} 1 / \cos \alpha=\left(1+z^{2}\right) /\left(1-z^{2}\right)$. 


\section{APÊNDICE B}

Hyperspherical Formulation of Inpurity-Bomud

Excitons in Semiconcluctors 


\title{
Hyperspherical formulation of impurity-bound excitons in semiconductors
}

J. J. De Groote and J. E. Hornos

Instituto de Física e Química de Sao Carlos, Universidade de Sao Paulo, 13560 Sao Carlos, Sao Paulo, Brazil

\section{H. T. Coelho}

Departamento de Fisica, Universidade Federal de Pernambuco, 50739 Recife, Pernambuco, Brazil

\author{
C. D. Caldwell \\ Department of Physics, University of Central Florida, Orlando, Florida 32816 \\ (Received 24 February 1992)
}

\begin{abstract}
A hyperspherical formalism is shown to be an appropriate approach for the investigation of the three-particle complex corresponding to an exciton bound to a Coulomb center in a semiconductor. The ground-and excited-state potential curves and binding energies are calculated as a function of the mass ratio of the hole and electron, and the concept of a critical mass is discussed. The results that we have obtained are in very good agreement with variational calculations for several semiconductor materials.
\end{abstract}

\section{INTRODUCTION}

The properties of excitons bound to imperfections in semiconductors have been the subject of numerous theoretical $^{1-5}$ and experimental ${ }^{1,6-8}$ investigations. Bonding can occur to both neutral and ionized impurities. The simplest form of the complex has a molecularlike structure, which gives rise to several lines in the spectra in addition to those originating from the free exciton. More complicated behavior can arise from clustering ${ }^{9}$ of excitons into biexcitons or when the particles are subjected to axial external fields (quantum wells) ${ }^{10}$ or confinement fields (quantum dots). ${ }_{1}$ While a simple hydrogenic approach is adequate for the description of the properties of the free exciton, bond formation, clustering, and the presence of external fields requires a more sophisticated mathematical approach to treat the many-body effects.

Spherical coordinates in higher dimensions, normally called hyperspherical coordinates (HC), have been used frequently to solve few-body quantum problems in strong-interaction physics. ${ }^{12}$ The same approach has also been used in some instances in atomic and molecular physics $^{13-18}$ to obtain wave functions for the three-body problem. Normally the use of HC leads to a difficult problem of a coupled system of differential equations of infinite dimension. This difficulty becomes even more severe if Coulomb forces are present.

A well-established and, in essence, exact procedure for handling physical systems within the HC scheme is the hyperspherical adiabatic approach (HAA). The literature $^{12-18}$ is full of examples illustrating the use of the HAA from the atomic to the nuclear scale. This approach is similar to the Born-Oppenheimer approximation used to calculate vibrational potential curves for diatomic molecules. In the application of this technique, the Schrödinger equation in $\mathrm{HC}$ is solved at each fixed hyperradius $R$, the coordinate that describes the overall size of the system, to generate a family of effectivepotential curves $U_{\lambda}(R)$ : The integer $\lambda$ characterizes the set of orbital angular momenta of the various particles in the system. For short-range forces, $U_{\lambda}(R)$ is successfully obtained by diagonalization procedures. ${ }^{12}$ However, for long-range forces this procedure does not prove to be practical. The rapidly oscillating Jacobi polynomials, the hyperspherical harmonic functions that are the analytic solutions to the radial equation at small $R$, give rise to instabilities with slow convergence. Slow convergence also results from the fact that the asymptotic solutions are not easily expanded in terms of hyperspherical harmonics.

One approach to removing this difficulty is through the introduction of another variable, $z=\tan (\alpha / 2)$, where $\alpha$ is one of the hyperspherical angles in the three-body problem. The resulting differential equation in terms of $z$ can then be written in canonical form and does not require an expansion in oscillating harmonic functions. Further improvement of the convergence can be obtained by incorporating the properties of the differential equations in the small and large $R$ limits in the initial ansatz, thereby further simplifying the form of the canonical equation. Once $U_{\lambda}(R)$ is known, the radial equation for $R$ can be solved to obtain the eigenenergies of the system.

The aim of this paper is to introduce the HAA to the realm of problems in exciton physics and to show that it forms an appropriate framework for the understanding of the difficulties outlined above. The specific system that will be addressed is the bonding of the exciton to an ionized donor. In the course of the calculation we will make use of a recently proven set of basic inequalities ${ }^{19}$ that provide a lower bound-upper bound relation for the ground-state energies of this system. The concept of a critical mass is also discussed in light of the exact method presented in this work. It seems that its existence is linked to the accuracy of the theoretical calculation.

This paper is organized in the following manner. In Sec. Il we present the general formalism as it exists in the 
literature. A method for obtaining potential curves similar to the Born-Oppenheimer approximation is given in Sec. 11I. The calculation of the binding energies and wave functions will be the subject of Sec. IV. Finally, in Sec. $V$ we will give the conclusions. Two appendixes are *also included.

\section{THE EFFECTIVE-MASS MODEL IN THE HYPERSPHERICAL APPROACH}

The binding of an exciton to an ionized impurity can be properly described in the effective-mass model by the Schrödinger equation for the motion of the electron-hole pair in the field of a fixed-impurity center of change $Z e$, as

$$
\begin{aligned}
{\left[-\frac{\hbar^{2}}{2 m_{e}^{*}} \nabla_{e}^{2}-\frac{\hbar^{2}}{2 m_{h}^{*}} \nabla_{h}^{2}-\frac{Z e^{2}}{\epsilon\left|\mathbf{r}_{e}\right|}+\frac{Z e^{2}}{\epsilon\left|\mathbf{r}_{h}\right|}\right.} \\
\left.-\frac{e^{2}}{\epsilon\left|\mathbf{r}_{e}-\mathbf{r}_{h}\right|}-E\right] \psi\left(\mathbf{r}_{e}, \mathbf{r}_{h}\right)=0 .
\end{aligned}
$$

The effects of the many-body interactions are represented in the effective masses of the electron $m_{e}^{*}$ and the hole $m_{h}^{*}$ and in the static dielectric constant $\epsilon$ of the material. Here $r_{e}$ and $r_{h}$ are the electron and hole distances from the ionized donor impurity, respectively. Instead of using the independent particle coordinates $\left(r_{e}, \theta_{e}, \varphi_{e}\right)$ and $\left(r_{h}, \theta_{h}, \varphi_{h}\right)$, we solve Eq. (1) by making a transformation to hyperspherical coordinates, as shown in a general procedure given in Appendix A. In Appendix $A$, if we set $m_{1} \rightarrow \infty, m_{2}=m_{e}^{*}, m_{3}=m_{h}^{*}, q_{1}=Z e, q_{2}=-e$, and $q_{3}=e$, and define $m=m_{h}^{*} / m_{e}^{*}$, we obtain

$$
\left[\frac{\partial^{2}}{\partial R^{2}}+\frac{\frac{1}{4}-U(R, \Omega)}{R^{2}}+\varepsilon\right] \Psi(R, \Omega)=0,
$$

where $\Psi$, the renormalized wave function, and $U(R, \Omega)$ are defined in Appendix A. by

$$
\begin{aligned}
\mathcal{D}(\alpha, \theta)= & -\left(\frac{m_{e}^{*}}{\mu}\right)^{1 / 2} \frac{Z e^{2}}{\epsilon \sin \alpha}+\left(\frac{m_{h}^{*}}{\mu}\right)^{1 / 2} \frac{Z e^{2}}{\epsilon \cos \alpha} \\
- & \left(\frac{m_{e}^{*}}{\mu}\right)^{1 / 2} \frac{e^{2}}{\epsilon}\left[\sin ^{2} \alpha+\frac{\cos ^{2} \alpha}{m}\right. \\
& \left.-\frac{\sin (2 \alpha) \cos \theta}{\sqrt{m}}\right)^{-1 / 2},
\end{aligned}
$$

where

$$
\hat{v}(\alpha, \theta)=\left(2 \mu / \hbar^{2}\right) \hat{V}(\alpha, \theta) .
$$

We will seek solutions to Eq. (2) that are characterized by a constant-total angular momentum $L$ and projection $M$. Then, the solution to the hyperspherical equations proceeds in the following way. First, a set of eigenvalues for the operator $U$ is determined as a function of the radial coordinate $R$ according to

$$
U(R, \Omega) \Phi_{\lambda}(R, \Omega)=U_{\lambda}(R) \Phi_{\lambda}(R, \Omega),
$$

where the index $\lambda$ denotes the set of integer labels $\left(L, M, l, l_{e}, l_{h}\right)$. The integers $l, l_{e}$, and $l_{h}$ derive from the characterization of the angular solution of Eq. (5) in the case of $R=0$. In this limit the equation can be solved ex. actly, ${ }^{13}$ yielding eigenvalues

$$
U_{\lambda}(0)=\left(2 l+l_{e}+l_{h}+2\right)^{2},
$$

and eigenfunctions

$$
\begin{aligned}
& \Phi_{\lambda}(0, \Omega)=\sum_{l_{e}^{\prime}, l_{h}^{\prime}}(\sin \alpha)^{l_{e}^{\prime}+1}(\cos \alpha)^{l_{h}^{\prime}+1} \\
& \quad \times P_{l}^{l_{e}^{\prime}+1 / 2, l_{h}^{\prime}+1 / 2}(\cos (2 \alpha)) \mathcal{Y}_{l_{e}^{\prime} l_{h}^{\prime}}^{L M}\left(\hat{\xi}_{e}, \hat{\xi}_{h}\right),
\end{aligned}
$$

where $P$ and $\mathscr{Y}$ are the Jacobi polynomials and the generalized spherical harmonics, respectively. Once the eigensolutions of Eq. (5) have been obtained, they can then be used as a basis for the expansion of the wave function $\Psi$ as

$$
\Psi(R, \Omega)=\sum_{\lambda} F_{\lambda}(R) \Phi_{\lambda}(R, \Omega)
$$

In the second step we return to the Schrödinger equation in order to obtain a set of radial equations

$$
\left[\frac{d^{2}}{d R^{2}}+\frac{\frac{1}{4}-U_{\lambda}(R)}{R^{2}}+\varepsilon\right] F_{\lambda}(R)+\sum_{\lambda^{\prime}} W_{\lambda \lambda^{\prime}}(R) F_{\lambda^{\prime}}(R)=0 \text {, }
$$

where

$$
W_{\lambda \lambda^{\prime}}(R)=2 P_{\lambda \lambda^{\prime}}(R) \frac{d}{d R}+Q_{\lambda \lambda^{\prime}}(R),
$$

and

$$
\begin{aligned}
& \left.P_{\lambda \lambda^{\prime}}(R)=\left\langle\Phi_{\lambda}\right| \frac{d \Phi_{\lambda^{\prime}}}{d R}\right)=\int_{\Omega} \Phi_{\lambda}^{*} \frac{d \Phi_{\lambda^{\prime}}}{d R} d \Omega \\
& Q_{\lambda \lambda^{\prime}}(R)=\left\langle\Phi_{\lambda} \mid \frac{d^{2} \Phi_{\lambda^{\prime}}}{d R^{2}}\right\rangle=\int_{\Omega} \Phi_{\lambda}^{*} \frac{d^{2} \Phi_{\lambda^{\prime}}}{d R^{2}} d \Omega
\end{aligned}
$$

Solutions to the set of Eq. (9) can be obtained within various approximations. The simplest of these, the extreme adiabatic approximation (EAA), neglects all coupling terms given by Eqs. (10)-(12). The energies calculated in this approximation form a lower bound to the exact value. ${ }^{19}$ Inclusion of the diagonal term $W_{\lambda \lambda}(R)$ gives the so-called uncoupled adiabatic approximation (UAA). While satisfactory results can be obtained in either of these limits, a systematic improvement can be achieved by the successive inclusion of additional off-diagonal coupling terms, giving rise to the coupled adiabatic approximation (CAA). The ground-state binding energies obtained in these adiabatic approximations satisfy the basic inequalities $^{19}$

$$
\varepsilon_{\mathrm{EAA}} \leq \varepsilon \leq \varepsilon_{\mathrm{CAA}} \leq \varepsilon_{\mathrm{UAA}}
$$




\section{POTENTIAL CURVES}

AND CHANNEL FUNCTIONS

In the solution of Eq. (5) to obtain the eigenpotentials $U_{\lambda}(R)$, it is necessary to develop an efficient procedure for handling the numerical calculations such that the $U_{\lambda}(R)$ 's are precisely obtained for the entire range of $R$. In order to achieve this in an efficient way, we begin by examining the kinetic term of Eq. (A7). That term has poles at $\alpha=0, \pi / 2$ that are independent of the masses of the system. On the other hand, the Coulomb interaction therm, given by Eq. (3), has poles at $\alpha=0$ and at $(\theta, \alpha)=\left(\pi, \alpha_{+}\right)$and $\left(0, \alpha_{-}\right)$, where $\tan \alpha_{ \pm}=\eta_{ \pm}$. In Appendix $A$, if we set $m_{1}=\infty$, we obtain $\alpha_{+}=\pi / 2$ and $\tan \alpha_{-1}=1 / \sqrt{m}$. Let us define $\alpha_{-}=\alpha_{c}$. Then, the porential curves are obtained by considering regular solutions in the two regions $0 \leq \alpha \leq \alpha_{c}$ and $\alpha_{c} \leq \alpha \leq \pi / 2$ and imposing continuity conditions at those boundary points. Notice that the presence of the poles at $\alpha=0$ and $\alpha=\pi / 2$ corresponds to the ionization limits, either the electron is at infinity or the hole is at infinity.

In this kind of investigation it is important to select the appropriate angles and the corresponding harmonic for use as a basis for the problem. There exist different ways to select those angles: these have been well studied by Smirnov and Shitikova. ${ }^{20}$ Each possibility is identified with a possible chain in which the group $O(6)$ can have its representation decomposed. As we seek solutions that are characterized by the total value of the angular momentum $L$, the most appropriate basis for the expression of the angular dependence is given by the twoparticle spherical harmonics. Thus, we have chosen the chain

$$
\begin{aligned}
& O(6) \supset O(3) \times O(3) \supset O(3) \supset O(2) \\
& \begin{array}{lllll}
1 & 1 & 1
\end{array} \\
& \left\{n l_{e} \ell_{h}\right\}\left\{\ell_{c}\right\} \quad\left\{l_{h}\right\} \quad\{L\} \quad\{M\} \text {, }
\end{aligned}
$$

which corresponds to the use of the three-dimensional two-particle spherical harmonics. The channel functions, i.e., the solutions to the eigenvalue equation (5) for each value of $R$, are then written as

$$
\begin{aligned}
\Phi_{\lambda}(R, \Omega)=\sum_{l_{e}, l_{h}} & (\sin \alpha)^{l_{e}+1}(\cos \alpha)^{l_{h}+1} \\
& \times \mathcal{Y}_{l_{e} l_{h}}^{L M_{e}}\left(\hat{\xi}_{e}, \hat{\xi}_{h}\right) g_{l_{e} l_{h}}^{\lambda}(R, \alpha),
\end{aligned}
$$

where $\mathcal{Y}_{l_{e} l_{h}}^{L M}$ is the usual two-particle coupled-orbital angular-momentum function $\left(\left|l_{e}-l_{h}\right| \leq L \leq l_{e}+l_{h}\right.$, and $\left.M=m_{e}+m_{h}\right)$. The basis functions $\mathscr{S}_{l_{e} l_{h}}^{\lambda}(R, \alpha)$ at each $R$ are obtained from the solution of Eq. (5). The strong singularities at $\alpha=0, \pi / 2$ are smoothed by the presence of the functions $\sin \alpha$ and $\cos \alpha$ in Eq. (15).

The motivation for the choice given by Eq. (15), instead of other possibilities (such as Euler angles ${ }^{14}$ or the canonical form used in nuclear physics ${ }^{12}$ ), is the following. The decomposition in the two $\mathrm{O}(3)$ groups preserves the individuality of the effective particles with respect to the angular motion in the $\left(\theta_{e}, \varphi_{e}\right),\left(\theta_{h}, \varphi_{h}\right)$ manifolds. However, the introduction of the variables $\alpha$ and $R$ will account for the collective effects.

We note that the function $\varrho_{l_{l} l_{h}}^{\lambda}(R, \alpha)$ in Eq. (15) was not further expanded in any basis. In this respect our approach differs from the usual techniques which are based on an expansion using orthonormal polynomials $12,14,16,17$ or another ${ }^{14}$ set of functions with faster convergence properties. As discussed elsewhere, ${ }^{13}$ our approach seems to be the most appropriate for long-range forces. For Coulombic systems the use of other approaches generates a number of problems, such as slow convergence, incorrect asymptotic behavior, nonorthonormal sets, etc. In order to avoid these problems we shall solve directly the infinite set of differential equations obtained by substituting Eq. (18) into Eq. (11), namely

$$
\left\{\frac{d^{2}}{d \alpha^{2}}+2\left[\left(l_{e}+1\right) \cot \alpha-\left(l_{h}+1\right) \tan \alpha\right] \frac{d}{d \alpha}-U_{\lambda}(R)-\left(l_{e}+l_{h}+2\right)^{2}\right\} g_{l_{e} l_{h}^{\prime}}^{\lambda}(R, \alpha)-R \sum_{l_{l}^{\prime}, l_{h}^{\prime}} \delta_{l_{e} l_{h} l_{e}^{\prime} l_{h}^{\prime}}^{L M}(\alpha) g_{l_{e}^{\prime} l_{h}^{\prime}}^{\lambda}(R, \alpha)=0,
$$

$$
\begin{aligned}
& \text { where } \\
& \nabla_{l_{e} l_{h} l_{e}^{\prime} l_{h}^{\prime}}^{L M}(\alpha)=(\sin \alpha)^{l_{e}^{\prime}-l_{e}}(\cos \alpha)^{l_{h}^{\prime}-l_{h}}\left\langle\mathcal{Y}_{l_{e} l_{h}^{L}}^{L M}|\hat{v}| Y_{l_{e}^{\prime} l_{h}^{\prime}}^{L M}\right\rangle
\end{aligned}
$$

The explicit expression for $\langle\hat{v}\rangle$ is given in Appendix B. In the solution of Eq. (16) it is necessary to keep in mind the symmetries imposed in the hyperspherical harmonic functions $S_{l_{e} I_{h}}^{\lambda}(R, \alpha)$. A discussion of this point is given, for example, in Refs. 12 and 13. Equation (16) is solved in the region $0 \leq \alpha \leq \alpha_{c}$. A totally analogous procedure applied to the region $\alpha_{c} \leq \alpha \leq \pi / 2$ leads to a similar equation.

We demonstrated earlier ${ }^{13}$ that instabilities which hamper the successful solution of the eigenvalue equation for long-range forces can be avoided by the introduction of another variable $z=\tan (\alpha / 2)$. This allowed us to rewrite the eigenvalue equation for $U_{\lambda}(R)$ in the "canonical" form, i.e., all coefficients are simple rational functions of the variable $z$. Therefore, a power series solution could be implemented via a finite recursion formula. We will exploit the same technique in the solution of the eigenvalue equation, Eq. (16). To this end we introduce the variable $z=\tan (\alpha / 2)$ in the two regions. The two equations in each region are analogous and take the form

$$
\begin{aligned}
{\left[\frac{d^{2}}{d z^{2}}+\mathcal{P}_{l_{e} l_{h}}(z) \frac{d}{d z}\right.} & \left.+Q_{l_{e} l_{h}}\right] g_{l_{e} l_{h}}^{\lambda}(R, z) \\
& +R \sum_{l_{e}^{\prime} l_{h}^{\prime}} R_{l_{e} l_{e}^{\prime} l_{h} l_{h}^{\prime}} g_{l_{e}^{\prime} l_{h}^{\prime}}^{\lambda}(R, z)=0,
\end{aligned}
$$

where 


$$
\begin{aligned}
P_{l_{e} l_{h}}(z)= & \frac{2}{1+z^{2}}\left[z+\frac{\left(l_{e}+1\right)\left(1-z^{2}\right)}{z}\right. \\
& \left.-\frac{4\left(l_{h}+1\right) z}{1-z^{2}}\right], \\
Q_{l_{e} l_{h}}(z)= & -4\left[\left(l_{e}+l_{h}+2\right)^{2}+U_{\lambda}(R)\right] /\left(1+z^{2}\right)^{2} \\
& +\left[\frac{m_{e}^{*}}{\mu}\right]^{1 / 2} \frac{Z e^{2}}{\epsilon} \frac{2 R}{z\left(1+z^{2}\right)} \\
& -\left(\frac{m_{h}^{*}}{\mu}\right]^{1 / 2} \frac{Z e^{2}}{\epsilon} \frac{4 R}{\left(1+z^{2}\right)\left(1-z^{2}\right)},
\end{aligned}
$$

and

$$
\begin{aligned}
R_{\left.l_{e} l_{e}^{\prime} l_{h}^{\prime} l_{h}^{(} z\right)=} & \frac{4}{\left(1+z^{2}\right)^{2}}\left|\frac{2 z}{1+z^{2}}\right|^{l_{e}^{\prime}-l_{e}} \\
& \left.\times\left(\frac{1-z^{2}}{1+z^{2}}\right)^{l_{h}^{\prime}-l_{h}} \mid \frac{m_{e}^{*}}{\mu}\right)^{1 / 2} \frac{e^{2}}{\epsilon} \Delta_{l_{e} l_{e}^{\prime} l_{h} l_{h}^{\prime}}
\end{aligned}
$$

Equation (18) can be solved in each region of space through a series expansion in the variable $z$. In contrast to the simpler helium system with two electrons, care must be taken in evaluating the series, as the angle $\alpha_{c}$ can become large for large values of the mass ratio. This implies that it may be necessary to use many more terms in the expansion to ensure convergence in one or the other region.

The solution of Eq. (18) involves the truncation of the expansion in the angular momenta $l_{e}$ and $l_{h}$. However, in contrast to the situation obtained in atomic physics, the formation of the bound state for the system described here is critically dependent on the presence of the correlation. Thus, it is necessary to include many more terms in the angular expansion than the three or four which typically deliver good results in the more familiar system.

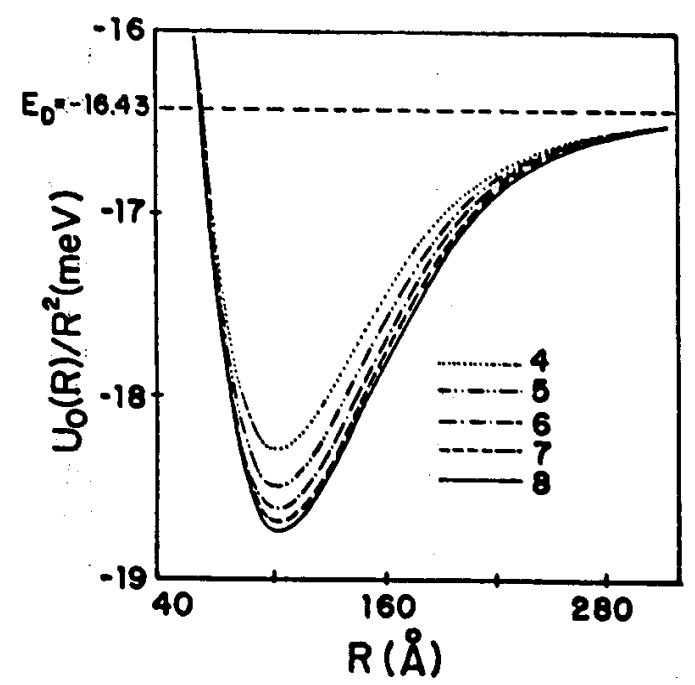

FIG. 1. Potential curves as a function of the number of angular channels for $\mathrm{ZnSe}$. Graphs here are given for 4-8 channels. Note that convergence requires the use of a large number of channels in the calculation. The deeper the curve the larger the number of channels required.
In order to understand the critical role that correlation plays in the bonding of the exciton to the impurity, we compare it with the helium problem. ${ }^{13}$ In the case of helium, a zeroth-order approximation in which the particle-particle correlation is totally neglected produces a bound state. In the case of the exciton, the neglect of the correlation (electron-hole) interaction produces a scattering system in which one particle is captured by the impurity and the other is free in the crystal. When the electron-hole interaction is turned on and a systematic inclusion of higher- and higher-angular-momentum channels is carried out, the minimum of the potential curve is uniformly lowered. As a result, the three-body system is bound together. This can be seen clearly in Fig. 1, which gives the convergence of the potential for the semiconductor $\mathrm{ZnSe}$ as a function of the number of angularmomentum channels used. A considerable change in the potential minimum is still taking place for the jump from four to five channels. Typically, we use eight angularmomentum channels in our calculations.

Another effect of the truncation of the number of angular-momentum channels included in the expansion is the violation of the inequalities given by Eq. (13). The validity of these inequalities is derived on the basis of a variational principle which assumes completeness of the angular-momentum expansion. If the contributions of only one or two channels in the expansion closely represent the minimum of the potential curve, then the inequalities remain valid even when truncation is performed. However, if convergence is not achieved in the expansion, then the completeness requirement is not even approximately fulfilled, and the inequalities are violated.

Figure 2 shows the potential curves for several different semiconductors having different electron-hole mass ratios. The overall depth and location of the minima of these curves vary in a uniform fashion with respect to the ratio $m$ of the hole mass to the electron mass. In particular, a detailed study of this variation yields the re-

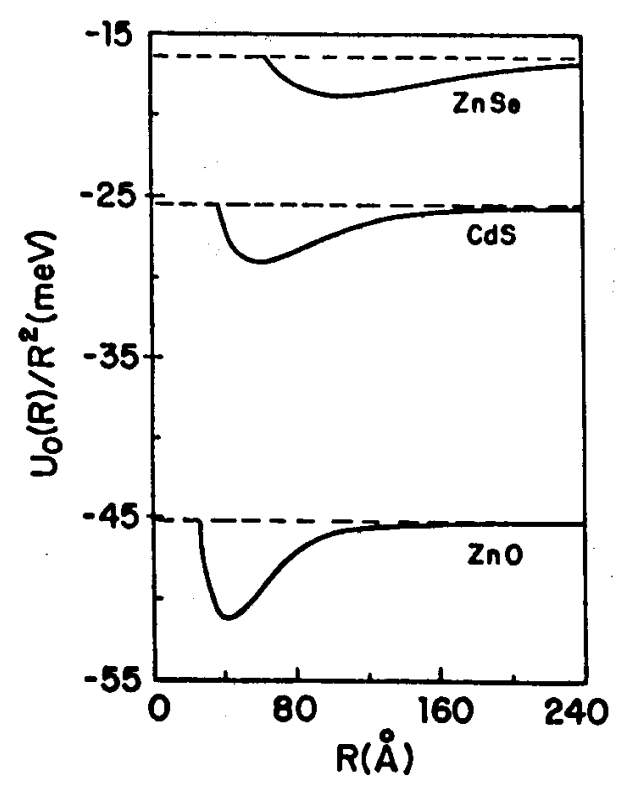

FIG. 2. Potential curves for selected semiconductors having different effective masses. The limits for large $R$ correspond to the donor energies $E_{D}$ when the hole is moving freely. 
ull that there is a nearly linear relationship between both and $U_{0}(R) / R^{2}$ at $R=R_{\min }$ and $m$. The influence of ais behavior will be apparent in the results obtained for whe binding energies in the next section.

\section{BOUND STATE ENERGIES AND WAVE FUNCTIONS}

In the EAA the radial equations, Eq. (9), reduce to a single uncoupled equation for each of the curves $U_{\lambda}(R)$ of interest. Using potential curves $U_{\lambda}(R)$, of the type given in Fig. 1 as input to Eq. (9), we calculated binding energies in the EAA. Table I gives the results that we have obtained for several prominent semiconductors. A comparison of our results with earlier variational calculations is also given. We note that in all cases, the binding energies are a small fraction of the threshold energy if we compare them with realistic molecular systems like $\mathrm{H}_{2}{ }^{+}$ or $\mathrm{D}_{2}{ }^{+}$. This places considerable demands on the requirements for saturation in the angular-momentum expansion. In fact, in the case of InSb, the sample with the beaviest-hole mass ratio, the result violates the inequality expressed in Eq. (13). It is, indeed, higher than the $10.30 \%$ clearly signaling lack of convergence in the expansion.

When this HC method is compared to other apgroaches with regard to the influence of the hole mass, ve see that it is, in some sense, complementary to the variational approach. In the $\mathrm{HC}$ case, larger hole masses require the inclusion of more angular-momentum components, thereby introducing difficulties and increasing errors. In a typical variational calculation, the region most difficult to handle is the region closer to the instability (light-hole mass) in which the expansion basis must contain both bound and scattering states. The last two columns can then be interpreted in light of Eq. (13) as establishing limits for the lower and upper bounds, respectively, on the binding energies. Within the limitations of the HC method, however, the calculated energies agree reasonably well with both the experimental and the theoretical resi: ts. The binding energies as a function of the mass ratio $m$ in Fig. 3 are plotted. The energies are given as percentages of the binding energy of the donor in the limit of large $J$ for the potential curve. For large values of $m$ the system approaches that of the $\mathrm{H}_{2}^{+}$molecule. For the range of values $2 \leq m \leq 6$ corresponding to the common semiconductors, the fractional binding ener-

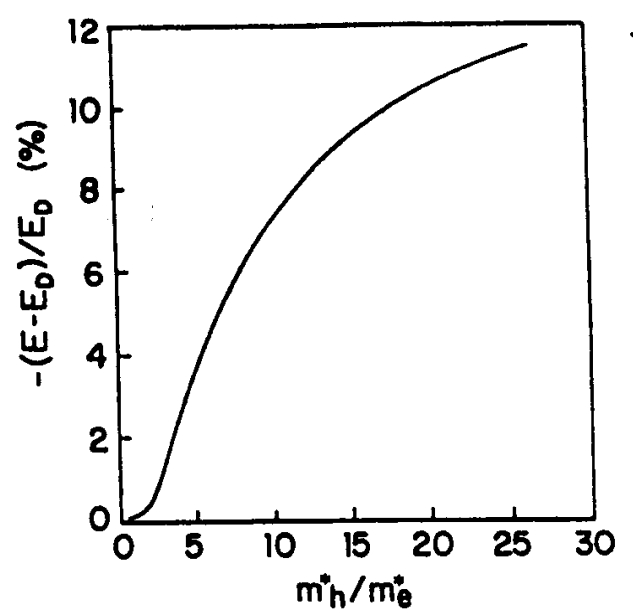

FIG. 3. Binding energy of the exciton-ionized donor complex as a function of the hole-electron mass ratio. $E_{D}$ is the energy of the donor when the hole is at $R=\infty$.

gy shows a very nearly linear variation with respect to $m$. For values smaller than 2.0 , the fractional binding energy drops abruptly. This implies that other interactions which have been neglected in this calculation will likely perturb the system and can cause dissociation. Thus, bound states having energy this low may not be observed experimentally in semiconductors haviag holes of small mass.

The occurrence of bonding of the exciton to the ionized impurity is intimately connected to the value of the mass ratio $m$. This is easy to visualize $\mathrm{x}$ hen one notes that the bonding of the exciton to the ionized impurity will lead to a localization of the wave function in the region of the center. As the bonding is largely determined by the correlation between the electron and the hole, this wave function will have a much greater extent than is generally the case for the ground states of the atomic systems. As the mass of the hole increases, a transition takes place from a local mass region, in which the molecular behavior is dominant, to a delocalized sector, in which the wave function is spread. In order to understand the delocalization pattern, we have plotted in Fig. 4 the radial wave function $\left|F_{0}(R)\right|^{2}$ for several values of $m$. As $m$ decreases, the maximum value of the wave function is lowered, and it tends to spread to larger values of $\boldsymbol{R}$. Another way of looking at this behavior is given in Fig. 5, in which the mean value of the hyperspherical values, $\langle R\rangle$, is plotted as a function of $m$. For small values of $m$

TABLE I. Binding energies for several semiconductors. $E_{D}$ is the dissociation energy. The indices refer to variational calculations. The static dielectric constants $\epsilon$ are taken from Ref. 3.

\begin{tabular}{crcccccccc}
\hline \hline Material & $\epsilon$ & $m_{e}^{*}$ (amu) & $m_{h}^{*}$ (amu) & $m_{e}^{*} / m_{h}^{*}$ & $E_{D}(\mathrm{meV})$ & $E-E_{D}(\mathrm{meV})$ & $\left(E-E_{D}\right) / E_{D}(\%)$ \\
\hline InSb & 16.80 & 0.02 & 0.30 & 0.067 & 0.9641 & 0.0893 & $0.0993^{*}$ & 9.26 & $10.30^{\circ}$ \\
$\mathrm{ZnSe}$ & 9.10 & 0.10 & 0.60 & 0.167 & 16.43 & 0.751 & $0.676^{*}$ & 4.57 & $4.12^{\circ}$ \\
CdS & 10.33 & 0.20 & 1.00 & 0.20 & 25.50 & 0.918 & $0.791^{\mathrm{b}}$ & 3.60 & $3.10^{\mathrm{b}}$ \\
$\mathrm{ZnO}$ & 8.50 & 0.24 & 1.14 & 0.21 & 45.19 & 1.514 & $1.220^{\mathrm{b}}$ & 3.35 & $2.70^{\mathrm{b}}$ \\
CdSe & 10.66 & 0.13 & 0.59 & 0.22 & 15.56 & 0.486 & $0.348^{*}$ & 3.12 & $2.23^{\mathrm{a}}$ \\
\hline \hline
\end{tabular}

Seet Ref. 3.

See Ref. 4. 


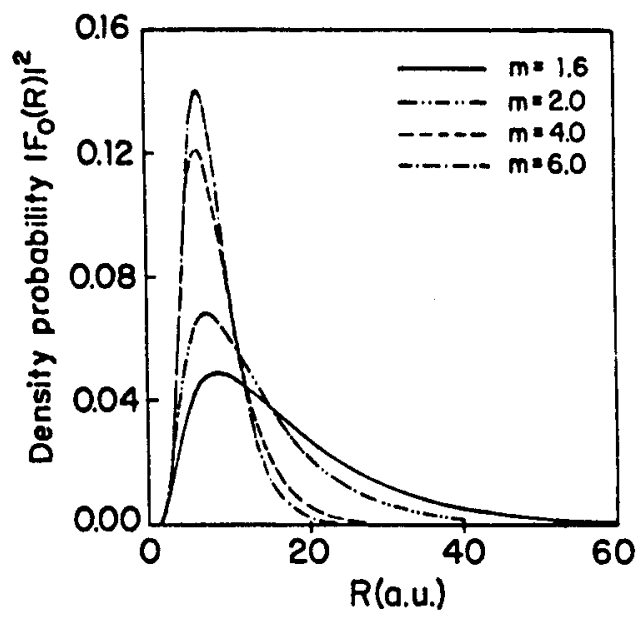

FIG. 4. Probability density $\left|F_{\lambda}(R)\right|^{2}$ for materials having different hole-electron mass ratios and the same dielectric constant. Note how the effect of the correlation distends the wave function as the hole mass become lighter.

the mean value increases sharply, while for larger values of $m$ the mean value approaches a constant value, in keeping with the anticipated molecular like behavior. For $m=5$, the Coulombic system reaches its minimum size. This corresponds to the $m$ value close to that for those semiconductors for which bound excitons have been observed.

Several values for the so-called "critical mass" at which bond formation will occur are found in the literature. ${ }^{1,2,22,23}$ It seems that the concept of a critical mass is inextricably linked to the accuracy with which the calculation can be performed in the mass region around the critical mass.

We should also mention here the effect of the nonadiabatic couplings. The inclusion of the $W_{\lambda \lambda^{\prime}}(R)$ terms [see Eq. (10)] will certainly increase the binding energy. It will certainly make quantitative changes in the other ob-

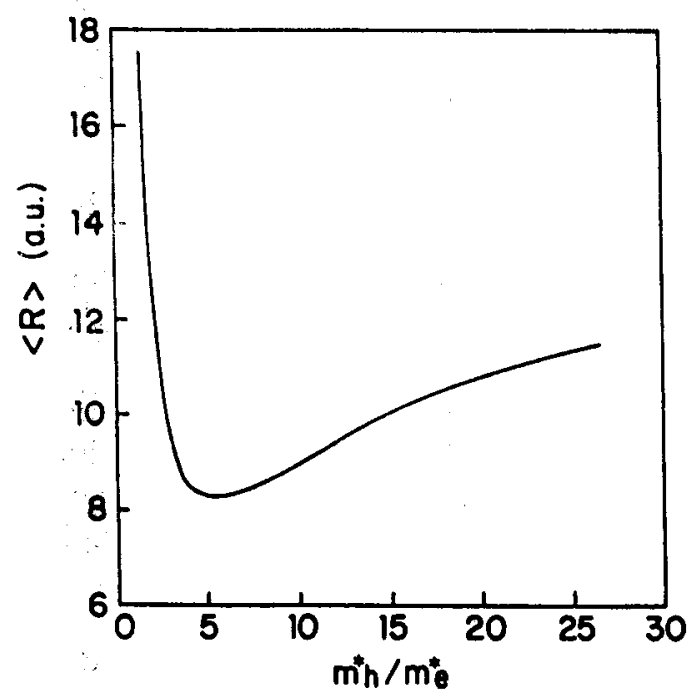

FIG. 5. Mean value of the hyperspherical radius in the effective atomic unit defined in the text. For small values of the mass ratio the size of the system increases drastically. Most of the semiconductors lie near the minimum of this curve. servables as a function of $m$. However, the basic arguments discussed above are, of course, not dependent on the inclusion of such nonadiabatic corrections but on the analytical properties of our equations.

\section{CONCLUSIONS}

In this work we have shown that the mathematical ap. proach of the use of hyperspherical coordinates in the adiabatic approximation (HAA) is successful in describ. ing excitons bound to imperfections in semiconductors even though the bonding is weak. The results that we ob. tain in the simplest EAA approach to the calculation are in very good agreement with variational calculations for several semiconductor materials. In light of the fact that the binding energies calculated in the EAA should represent a lower bound for the ground-state energy of the system, our results are a determination of this lower bound for a number of semiconductors. Only for those semiconductors for which the truncation of angularmomentum channels included in the calculation limits convergence is the inequality equation (13) not valid. For this case the EAA result is not a lower bound.

The concept of an overall size of the bound state contained in the definition of the hyper-radius $R$ allows us to describe the bond formation through the localization of the hole and electron in the vicinity of the impurity. This overall size is shown to be dependent on the ratio $m$ of the mass of the hole to that of the clectron. This behavior is clearly illustrated in the behavior of the binding energies (Fig. 3) and the mean value of the hyper-radius $(R)$ (Fig. 5) as a function $m$. It is apparent from the localized behavior of the wave function:;, as illustrated in Fig. 4, that bonding is possible for any value of the mass ratio $m$. This seems to imply that the emergence of a critical mass beyond which bonding cannot occur is an artifact of the computational accuracy and is not a fundamental limitation imposed by the physics of the problem. What appears in the calculation as a type of critical mass is actually a minimum in the size of the bound state, as reflected in the value of $\langle R\rangle$ at a specific value of $m$.

This approach to the use of hyperspherical coordinates in the calculation of bonding of excitons to charged impurities has been performed with all radial coupling terms neglected. Currently, more precise calculations including these terms are underway. While we anticipate that the results obtained with these terms included should be more accurate, the general behavior of the bonding and the localization should not be significantly altered. In particular, we expect the conclusion on the nonexistence of a critical mass to be unaffected by more accurate calculations that will be performed.

\section{ACKNOWLEDGMENTS}

One of the authors (H.T.C.) would like to thank Dr. Y. Nogami for useful discussions and the kind hospitality received at McMaster University where part of the manuscript was written. We would also like to thank Dr. M. Degani and Dr. O. Hipólito for useful discussions. This work was partially supported by Conselho Nacional de Desenvolvimento Científico e Tecnológico (CNPq), 
ıdação de Amparo à Pesquisa de Săo Paulo PESP), Fundaçào de Amparo à Ciência e Tecnologia Pernambuco (FACEPE), e Financiadora de Estudos e jetos (FINEP) (Brazilian agencies).

\section{APPENDIX A: THE HYPERSPHERICAL COORDINATES}

Jacobi coordinates is an appropriate set for the threedy problem. Let $\mathbf{r}_{i}$ be the coordinate of particle $i$ in e laboratory frame, which has mass $m_{i}$. Let $\rho_{1}$ be the ctor from particle 1 to particle 2 with reduced mass

$$
\mu_{1}=m_{1} m_{2} /\left(m_{1}+m_{2}\right)
$$

he second vector $\rho_{2}$ is from the center of mass (c.m.) of irticles 1 and 2 to particle 3 , with reduced mass

$$
\mu_{2}=\left(m_{1}+m_{2}\right) m_{3} /\left(m_{1}+m_{2}+m_{3}\right) \text {. }
$$

his procedure can easily be generalized to $N$-body sysms. $^{14}$ It is also useful to introduce a set of masseighted coordinates $\xi_{i}=\left(\mu_{i} / \mu\right)^{1 / 2} \rho_{i}$, where $\mu$ is an arbirary mass. The choice of Jacobi coordinates is not nique, ${ }^{14}$ but normally dictated by the nature of the physsal problem under consideration.

We can explicitly write down our choice of coordilates, used in this work, as

$$
\begin{aligned}
& \xi_{0}=\left(m_{1} \mathbf{r}_{1}+m_{2} \mathbf{r}_{2}+m_{3} \mathbf{r}_{3}\right) /\left(m_{1}+m_{2}+m_{3}\right), \\
& \xi_{1}=\left(\mu_{1} / \mu\right)^{1 / 2}\left(\mathbf{r}_{1}-\mathbf{r}_{2}\right), \\
& \xi_{2}=\left(\mu_{2} / \mu\right)^{1 / 2}\left(\frac{m_{1} \mathbf{r}_{1}+m_{2} \mathbf{r}_{2}}{m_{1}+m_{2}}-\mathbf{r}_{3}\right) .
\end{aligned}
$$

The Hamiltonian of our three-body system interacting via Coulomb forces is written as

$$
\begin{aligned}
H= & -\frac{\hbar^{2}}{2\left(m_{1}+m_{2}+m_{3}\right)} \nabla_{\xi_{0}}^{2}-\frac{\hbar^{2}}{2 \mu}\left(\nabla_{\xi_{1}}^{2}+\nabla_{\xi_{2}}^{2}\right) \\
& +V\left(\xi_{1}, \xi_{2}\right)
\end{aligned}
$$

where

$$
\begin{aligned}
\hat{V}(\alpha, \theta)= & {\left[\frac{\mu_{1}}{\mu}\right]^{1 / 2} \frac{q_{1} q_{2}}{\epsilon \sin \alpha}+\frac{m_{1}}{\epsilon \sqrt{\mu \mu_{1}}} q_{1} q_{3}\left[\sin ^{2} \alpha+\eta_{+}^{2} \cos ^{2} \alpha+\eta_{+} \sin (2 \alpha) \cos \theta\right]^{-1 / 2} } \\
& +\frac{m_{2}}{\epsilon \sqrt{\mu \mu_{1}}} q_{2} q_{3}\left[\sin ^{2} \alpha+\eta_{-}^{2} \cos ^{2} \alpha-\eta_{-} \sin (2 \alpha) \cos \theta\right]^{-1 / 2}
\end{aligned}
$$

and $\cos \theta=\hat{\boldsymbol{\xi}}_{1} \cdot \hat{\boldsymbol{\xi}}_{2}$. Note that

$$
\hat{v}(\alpha, \theta)=\left(2 \mu / \hbar^{2}\right) \hat{V}(\alpha, \theta) \text {. }
$$

\section{APPENDIX B: EVALUATION}

\section{OF THE CHANNEL-MIXING COEFFICIENTS}

To calculate the matrix elements of $v$ in Eq. (17), berance of the form of Eq. (5), we first need to evaluate the

$$
\begin{aligned}
V\left(\xi_{1}, \xi_{2}\right)= & \left(\frac{\mu_{1}}{\mu}\right)^{1 / 2} \frac{q_{1} q_{2}}{\epsilon \xi_{1}}+\frac{m_{1}}{\sqrt{\mu \mu_{1}}} \frac{q_{1} q_{3}}{\epsilon\left|\xi_{1}+\eta_{+} \xi_{2}\right|} \\
& +\frac{m_{2}}{\sqrt{\mu \mu_{1}}} \frac{q_{2} q_{3}}{\epsilon\left|\xi_{1}-\eta_{-} \xi_{2}\right|},
\end{aligned}
$$

and

$$
\begin{aligned}
& \eta_{+}=\left[m_{1}\left(m_{1}+m_{2}+m_{3}\right) / m_{2} m_{3}\right]^{1 / 2}, \\
& \eta_{-}=\left[m_{2}\left(m_{1}+m_{2}+m_{3}\right) / m_{1} m_{3}\right]^{1 / 2}
\end{aligned}
$$

The symbol $q_{i}$ stands for the particle electric charges and $\epsilon$ for the static dielectric constant. Note that the use of Jacobi coordinates separated the c.m. motion in the kinetic energy operator. In this way we can consider only the resulting Schrödinger equation in the c.m. At this point it is appropriate to introduce hyperspherical coordinates by defining a hyperspherical radius $R$

$$
R^{2}=\xi_{1}^{2}+\xi_{2}^{2},
$$

and a hyperspherical angle $\alpha$

$$
\begin{aligned}
& \xi_{1}=R \sin \alpha, \\
& \xi_{2}=R \cos \alpha, \quad 0 \leq \alpha \leq \pi / 2 .
\end{aligned}
$$

The Schrödinger equation, $H \psi=E \psi$ in hyperspherical coordinates can be written as ${ }^{12}$

$$
\left[\frac{\partial^{2}}{\partial R^{2}}-\frac{U(R, \Omega)-\frac{1}{4}}{R^{2}}+\varepsilon\right] \psi(R, \Omega)=0,
$$

where $\Psi(R, \Omega)$ is the normalized wave function,

$$
\Psi(R, \Omega)=R^{5 / 2} \sin \alpha \cos \alpha \psi(R, \Omega),
$$

$\varepsilon=\left(2 \mu / \hbar^{2}\right) E, E$ being the energy eigenvalue, and

$$
\Omega=\left\{a, \hat{\xi}_{1}=\left(\theta_{1}, \varphi_{1}\right), \hat{\xi}_{2}=\left(\theta_{2}, \varphi_{2}\right)\right\} .
$$

$U(R, \Omega)$, is given by

$U(R, \Omega)=-\frac{\partial^{2}}{\partial \alpha^{2}}+\frac{\hat{l}_{1}^{2}\left(\hat{\xi}_{1}\right)}{\sin ^{2} \alpha}+\frac{\hat{l}_{2}^{2}\left(\hat{\xi}_{2}\right)}{\cos ^{2} \alpha}+R \hat{v}(\alpha, \theta)$.

The quantities $\hat{l}_{i}^{2}\left(\hat{\xi}_{i}\right)(i=1$ and 2$)$ stand for the usual angular-momentum operators. Defining $v=\left(2 \mu / \hbar^{2}\right) V$, and $V(R, \Omega)=\hat{V}(\alpha, \theta) / R$, where

$$
\begin{aligned}
& \text { tensor } \\
& \begin{aligned}
\mathcal{T}_{l_{e} l_{e}^{\prime} l_{h} l_{h}^{\prime}}^{L M}(\alpha)= & \int d \hat{\xi}_{e} d \hat{\xi}_{h} Y_{l_{e}^{\prime} l_{h}^{\prime}}^{L M}\left(\hat{\xi}_{e}, \hat{\xi}_{h}\right)^{*} \\
& \times\left[\sin ^{2} \alpha+\eta_{-}^{2} \cos ^{2} \alpha-\eta_{-} \sin (2 \alpha) \cos \theta\right]^{-1 / 2} \\
& \times \mathcal{Y}_{l_{e} I_{h}}^{L M}\left(\hat{\xi}_{e}, \hat{\xi}_{h}\right) .
\end{aligned}
\end{aligned}
$$


Notice that the square-root term can always be written in the form

$$
f\left(1+t^{2}-2 t x\right)^{-1 / 2}, x=\cos \theta,
$$

where the expressions for $f$ and $t$ are given in Table II.

We also know by the definition of Legendre polynomials $^{21}$ that

$$
\left(1+t^{2}-2 t x\right)^{-1 / 2}=\sum_{L^{\prime}=0}^{\infty} t^{L^{\prime}} P_{L^{\prime}}(x), \quad|t|<1 .
$$

Because of the selection rules used below, the condition $|t|<1$ is not mandatory. Using the addition theorem for $P_{L}(x)$ and the fact that

$$
\begin{aligned}
Y_{l_{e} l_{h}}^{L M}\left(\hat{\xi}_{e}, \hat{\xi}_{h}\right)=\sum_{m_{e}, m_{h}} & \left(L M\left|l_{e} m_{e} l_{h} m_{h}\right\rangle\right. \\
& \times Y_{l_{e} m_{e}}\left(\hat{\xi}_{e}\right) Y_{l_{h} m_{h}}\left(\hat{\xi}_{h}\right),
\end{aligned}
$$

we obtain, after some straightforward algebra, the expres. sion

$$
\begin{aligned}
\langle\hat{v}\rangle= & -\left(\frac{m_{e}^{*}}{\mu}\right)^{1 / 2} \frac{Z e^{2}}{\epsilon \sin \alpha} \delta_{e} l_{e}^{\prime} l_{h} l_{h}^{\prime} \\
& +\left(\frac{m_{h}^{*}}{\mu}\right)^{1 / 2} \frac{Z e^{2}}{\epsilon \cos \alpha} \delta_{l_{e} l_{e}^{\prime} l_{h} l_{h}^{\prime}} \\
& -\left(\frac{m_{e}^{*}}{\mu}\right)^{1 / 2} \frac{e^{2}}{\epsilon} \Delta_{l_{e} l_{e}^{\prime} l_{h} l_{h}^{\prime}}(\alpha),
\end{aligned}
$$

TABLE 11. Expressions for $t$ and $f$ appearing in Eq. (B2).

\begin{tabular}{ccc}
\hline$\alpha$ interval & $f$ & $t$ \\
\hline $0 \leq \alpha \leq \alpha_{c}$ & $\left(\eta_{-} \cos \alpha\right)^{-1}$ & $\tan \alpha / \eta_{-}$ \\
$\alpha_{c} \leq \alpha \leq \pi / 2$ & $(\sin \alpha)^{-1}$ & $\eta_{-} \cot \alpha$ \\
\hline
\end{tabular}

where

$$
\Delta_{l_{e} l_{e}^{\prime} l_{h}^{\prime} l_{h}^{\prime}}(\alpha)=\sum_{L^{\prime}}(-1)^{L^{\prime}} C_{l_{e} l_{e}^{\prime} l_{h}^{\prime} l_{h}^{\prime L^{\prime}}} D_{L^{\prime}}(\alpha)
$$

and

$$
\begin{aligned}
C_{l_{e} l_{e}^{\prime} l_{h}^{\prime} l_{h}^{\prime}}^{L L^{\prime}}=(-1)^{L+L^{\prime}}\left[\left(2 l_{e}+1\right)\left(2 l_{e}^{\prime}+1\right)\left(2 l_{h}+1\right)\right. \\
\left.\times\left(2 l_{h}^{\prime}+1\right)\right]^{1 / 2} \\
\times\left(\begin{array}{lll}
l_{e} & l_{e}^{\prime} & L^{\prime} \\
0 & 0 & 0
\end{array}\right)\left[\begin{array}{lll}
l_{h} & l_{h}^{\prime} & L^{\prime} \\
0 & 0 & 0
\end{array}\right)\left\{\begin{array}{lll}
l_{e} & l_{h} & L \\
l_{h}^{\prime} & l_{e}^{\prime} & L^{\prime}
\end{array}\right\},
\end{aligned}
$$

where the usual $3-j$ and $6-j$ symbols appear. The $D_{L^{\prime}}$ terms are given by

$D_{L^{\prime}}(\alpha)=\left\{\begin{array}{l}\left(\tan \alpha / \eta_{-}\right)^{L^{\prime}}\left(\eta_{-} \cos \alpha\right)^{-1}, \quad 0 \leq \alpha \leq \alpha_{c} \\ \left(\eta_{-} \cot \alpha\right)^{L^{\prime}} / \sin \alpha, \quad \alpha_{c} \leq \alpha \leq \pi / 2,\end{array}\right.$

where $\tan \alpha_{+}=+\infty$ and $\tan \alpha_{-}=\tan \alpha_{c}=1 / \sqrt{m}$. In terms of the variable $z, D_{L^{\prime}}(z)$ can easily be obtained by noticing that $\tan \alpha=2 z /\left(1-z^{2}\right), \quad \cot \alpha=\left(1-z^{2}\right) / 2 z$, $(\sin \alpha)^{-1}=\left(1+z^{2}\right) / 2 z$, and $(\cos \alpha)^{-1}=\left(1+z^{2}\right) /\left(1-z^{2}\right)$.

28, 1812 (1983); T. K. Das, H. T. Coelho, and M. Fabre de la Ripelle, ibid. 26, 2281 (1982).

${ }^{13}$ J. E. Hornos, S. W. Mac Dowell, and C. D. Caldwell, Phys. Rev. A 33, 2212 (1986); H. T. Coelho, J. J. de Groote, and J. E. Hornos (unpublished).

${ }^{14}$ C. D. Lin and Xian-Hu Liu, Phys. Rev. A 37, 2749 (1988).

${ }^{15}$ C. D. Lin, Adv. Mod. Phys. 22, 77 (1986).

${ }^{16}$ J. H. Macek, J. Phys. B 1, 831 (1968).

${ }^{17}$ V. P. Brito, H. T. Coelho, and T. K. Das, Phys. Rev. A 40, 3346 (1989); S. K. Adhikari, V. P. Brito, H. T. Coelho, and T. K. Das, Nuovo Cimento (to be published).

${ }^{18}$ C. H. Greene, Phys. Rev. A 23, 661 (1981); V. Fano, Rep. Prog. Phys. 46, 97 (1983).

${ }^{19}$ H. T. Coelho and J. E. Hornos, Phys. Rev. A 43, 6379 (1991).

${ }^{20}$ Yu. F. Smirnov, and K. V. Shitikova, Fiz. Elem. Chastits At. Yadra, 8, 847 (1977) [Sov. J. Part. Nucl. 8 (4), 344 (1977)].

${ }^{21}$ Handbook of Mathematical Functions, edited by $M$. Abranowitz and I. A. Stegun, 3rd ed. (Dover, New York, 1965).

${ }^{22}$ Tsin-Fu Jiang, Solid State Commun. 74, 899 (1990).

${ }^{23}$ F. H. Gertler, H. B. Snodgrass, and L. Spruch, Phys. Rev. 172, 110 (1968). 


\section{APÊNDICE C}

General Theoretical Approach to Coulombic Threc-Body

Systems by the Hyperspherical Formalism 


\title{
General theoretical approach to Coulombic three-body systems by the hyperspherical formalism
}

\author{
H. T. Coelho \\ Departamento de Física, Universidade Federal de Pernambuco, 50739 Recife, Pernambuco, Brazil
}

\author{
J. J. De Groote and J. E. Hornos \\ Instituto de Física e Química de Sao Carlos, Universidade de Sao Paulo, 13560 \\ Sao Carlos, Sao Paulo, Brazil \\ (Received 13 August 1991; revised manuscript received 19 November 1991)
}

\begin{abstract}
Coulombic three-body systems are investigated using the hyperspherical adiabatic approach. By using a suitable variable $z=\tan (\alpha / 2)$ in the angular differential equation for the determination of the potential curves, we are able to obtain stable series-expansion solutions, valid for small and large values of the hyperspherical radius. The analysis of the mathematical singularities of the differential equations in the variable $z$ offers an insight into the physics of the problem and into the determination of stable converging solutions as well. In order to illustrate our investigation, we apply this study to several carefully chosen systems: $\mathrm{He}, d d \mu, d_{2}{ }^{+}$, and excitons bound to a Coulomb center in different semiconductors.
\end{abstract}

PACS number(s): $31.10 .+2,03.65 . G e$

\section{INTRODUCTION}

The study of three-body systems interacting via Coulomb forces is of great importance due to a large variety of physical systems found in atomic and molecular physics. In atomic physics the nuclear motion is neglected and the system is approximately described by the independent-electron model, where each electron is subject to an averaged screening potential. In the case of molecular physics, the traditional Born-Oppenheimer (BO) approximation is mostly used. Due to the large difference between the masses of protons and electrons, the BO approximation is well suited. In this approximation each internuclear separation is taken as fixed for the motion of the electron. These two approximate methods have their own limitations [1]. In the nonrelativistic case the hyperspherical approach [2] (HA) appears to be a method to treat three-body systems in a rigorous and transparent way. Normally the HA leads to a difficult solution of a coupled system of differential equations of infinite dimension. This problem becomes even more severe if Coulomb forces are present [3]. A wellestablished procedure $[4-10]$ to handle such physical systems within the HA is the hyperspherical adiabatic approach (HAA). This is similar to the BO approximation in diatomic molecules. The Schrödinger equation in hyperspherical coordinates is solved at each fixed hyperradius $R$ to generate a family of effective potential curves. These potential curves, similar to the molecular potentials, contain essential information about the structure of the three-body system. As a result, we are required to search for a procedure to obtain in an efficient way the potentials $U_{\lambda}(R)$ for the whole range of $R$ and for a variety of physical systems. The literature $[4-10]$ is full of works illustrating the use of the HAA from the atomic to the nuclear scale. Technical problems involve the process of obtaining $U_{\lambda}(R)$, mostly because of the slow convergence of the hyperspherical harmonic functions. For short-range forces, $U_{\lambda}(R)$ is successfully obtained by $\mathrm{di}$ agonalization procedures [8]. However for long-range forces this procedure appears not to be practical, mostly because of the highly oscillating Jacobi polynomials present in the hyperspherical-harmonic functicns, generating slow convergence. This slow convergence is also attributed to the fact that the asymptotic solutions are not easily expanded in terms of hyperspherical harmonics. A way to solve this difficulty is presented in this paper. Instead of the diagonalization procedure, ve look directly for solutions of the angular differential equations. This is partially accomplished by incorporating the properties of the differential equations in the small- as well as large- $R$ limits. These differential equations depend only on the hyperspherical angle $\alpha$ and contain trigonometric functions as coefficients of the derivatives. The hyperspherical radius $R$ is also present but taken as the adiabatic variable. In this paper we present a general formulation of the Coulombic three-body problem in the spirit of the authors of Ref. [7]. In that reference only the simplest system, the He atom, was considered. By developing an analytical solution of the angular differential equations in a power-series expansion in a suitable variable, namely, $z=\tan (\alpha / 2)$, we can transform the differential equations to a form where the Cauchy theorem can be applied. This basically implies that the above-mentioned coefficients become rational functions in the new variable z. Another resulting advantage of this transformation is to incorporate the topological properties of the mathematical singularities of the differential equations in order to obtain physical insights and generate stable converging solutions.

The next step after the precise determination of $U_{\lambda}(R)$ is to obtain the energies and wave functions of Coulombic three-body systems. That is achieved by solving a onedimensional Schrödinger-type equation in the variable $R$. In the HAA one makes use of a recently proved set of basic inequalities [12], which provide for the first time a 
lower-bound-upper-bound relation for the ground-state energies.

For the purpose of this work we use mass-weighted hyperspherical coordinates, to study Coulombic systems of arbitrary masses. Concerning the location of the singularities, we have carefully chosen several systems to illustrate our approach: $\mathrm{He}, d d \mu, d_{2}{ }^{+}$, and a three-particle complex corresponding to an exciton bound to a Coulomb center in a semiconductor. In the last case, results are calculated as a function of the mass ratio of the hole and electron, for several semiconductor materials.

In Sec. II we outline the HAA. In Sec. III we discuss how to obtain the potentials $U_{\lambda}(R)$. In Sec. IV we study several different Coulombic three-body systems, one of them (excitons) of varying mass. Finally, in Sec. $V$ we present our conclusions. Some sections are divided into subsections in order to make the article more pedagogical. An appendix is included for completeness of the sections.

\section{HYPERSPHERICAL ADIABATIC APPROACH}

\section{A. Hyperspherical formalism}

Jacobi coordinates form an appropriate set for the three-body problem. Let $\mathbf{r}_{i}$ be the coordinate of particle $i$ in the laboratory frame, which has mass $m_{i}$. Let $\rho_{1}$ be the vector from particle 1 to particle 2 with reduced mass $\mu_{1}=m_{1} m_{2} /\left(m_{1}+m_{2}\right)$. The second vector $\rho_{2}$ is from the center of mass (c.m.) of particles 1 and 2 to particle 3 , with reduced mass $\mu_{2}=\left(m_{1}+m_{2}\right) m_{3} /\left(m_{1}+m_{2}+m_{3}\right)$. This procedure can easily be generalized to $N$-body systems [11]. It is also useful to introduce a set of massweighted coordinates $\xi_{i}=\left(\mu_{i} / \mu\right)^{1 / 2} \rho_{i}$, where $\mu$ is an arbitrary mass. The choice of Jacobi coordinates is not unique [11], but normally dictated by the nature of the
physical problem under consideration.

We can explicitly set down our choice of coordinates used in this work:

$$
\begin{aligned}
& \xi_{0}=\left(m_{1} \mathbf{r}_{1}+m_{2} \mathbf{r}_{2}+m_{3} \mathbf{r}_{3}\right) /\left(m_{1}+m_{2}+m_{3}\right), \\
& \xi_{1}=\left(\mu_{1} / \mu\right)^{1 / 2}\left(\mathbf{r}_{1}-\mathbf{r}_{2}\right), \\
& \xi_{2}=\left(\mu_{2} / \mu\right)^{1 / 2}\left(\frac{m_{1} \mathbf{r}_{1}+m_{2} \mathbf{r}_{2}}{m_{1}+m_{2}}-\mathbf{r}_{3}\right) .
\end{aligned}
$$

The Hamiltonian of our three-body system interacting via Coulomb forces is written as

$$
H=-\frac{\hbar^{2}}{2\left(m_{1}+m_{2}+m_{3}\right)} \nabla_{\xi_{0}}^{2}-\frac{\hbar^{2}}{2 \mu}\left(\nabla_{\xi_{i}}^{2}+\nabla_{\xi_{2}}^{2}\right)+V\left(\xi_{1}, \xi_{2}\right),
$$

where

$$
\begin{aligned}
V\left(\xi_{1}, \xi_{2}\right)= & \left(\frac{\mu_{1}}{\mu}\right)^{1 / 2} \frac{q_{1} q_{2}}{\xi_{1}}+\frac{m_{1}}{\sqrt{\mu \mu_{1}}} \frac{q_{1} q_{3}}{\left|\xi_{1}+\eta_{+} \xi_{2}\right|} \\
& +\frac{m_{2}}{\sqrt{\mu \mu_{1}}} \frac{q_{2} q_{3}}{\left|\xi_{1}-\eta_{-} \xi_{2}\right|}
\end{aligned}
$$

and

$$
\begin{aligned}
& \eta_{+}=\left[m_{1}\left(m_{1}+m_{2}+m_{3}\right) / m_{2} m_{3}\right]^{1 / 2}, \\
& \eta_{-}=\left[m_{2}\left(m_{1}+m_{2}+m_{3}\right) / m_{1} m_{3}\right]^{1 / 2} .
\end{aligned}
$$

The symbols $q_{i}$ stand for the particle electric charges. Notice that the use of Jacobi coordinates separated the c.m. motion in the kinetic-energy operator. In this way we can consider only the resulting Schrödinger equation in the c.m. At this point it is appropriate to introduce hyperspherical coordinates by defining a hyperspherical radius $R$,

$$
R^{2}=\xi_{1}^{2}+\xi_{2}^{2},
$$

and a hyperspherical angle $\alpha$,

$$
\begin{aligned}
& \xi_{1}=R \sin \alpha, \\
& \xi_{2}=R \cos \alpha, 0 \leq \alpha \leq \pi / 2 .
\end{aligned}
$$

The Schrödinger equation $H \Psi=E \Psi$ in hyperspherical coordinates can be written as [7]

$$
\left\lfloor\frac{\partial^{2}}{\partial R^{2}}-\frac{U(R, \Omega)-\frac{1}{4}}{R^{2}}+\varepsilon\right\rfloor \psi(R, \Omega)=0,
$$

where $\varepsilon=\left(2 \mu / \hbar^{2}\right) E, E$ being the energy eigenvalue, and $\Omega=\left\{\alpha, \hat{\boldsymbol{\xi}}_{1}=\left(\theta_{1}, \varphi_{1}\right), \hat{\boldsymbol{\xi}}_{2}=\left(\theta_{2}, \varphi_{2}\right)\right\}$. Notice that $\psi(R, \Omega)$ is the renormalized wave function given by $\psi(R, \Omega)=R^{5 / 2} \sin \alpha \cos \alpha \Psi(R, \Omega)$. To define $U(R, \Omega)$, let us call

$$
U(0)=-\frac{\partial^{2}}{\partial \alpha^{2}}+\frac{\hat{l}_{1}^{2}\left(\hat{\xi}_{1}\right)}{\sin ^{2} \alpha}+\frac{\hat{l}_{2}^{2}\left(\hat{\xi}_{2}\right)}{\cos ^{2} \alpha} .
$$

The quantities $\hat{l}_{i}^{2}\left(\hat{\xi}_{i}\right) \quad(i=1,2)$ stand for the usual angular-momentum operators. Defining $v=\left(2 \mu / \hbar^{2}\right) V$ and $V(R, \Omega)=\hat{V}(\alpha, \theta) / R$, where

$$
\begin{aligned}
\hat{V}(\alpha, \theta)= & \left(\frac{\mu_{1}}{\mu}\right)^{1 / 2} \frac{q_{1} q_{2}}{\sin \alpha} \\
& +\frac{m_{1}}{\sqrt{\mu \mu_{1}}} q_{1} q_{3} /\left(\sin ^{2} \alpha+\eta_{+}^{2} \cos ^{2} \alpha\right. \\
& \left.+\eta_{+} \sin 2 \alpha \cos \theta\right)^{1 / 2} \\
& +\frac{m_{2}}{\sqrt{\mu \mu_{1}}} q_{2} q_{3} /\left(\sin ^{2} \alpha+\eta_{-}^{2} \cos ^{2} \alpha\right.
\end{aligned}
$$

and $\cos \theta=\hat{\xi}_{1} \cdot \hat{\xi}_{2}$, we finally have a useful relation:

$$
U(R, \Omega)=U(0)+R^{2} v(R, \Omega)=U(0)+R \hat{v}(\alpha, \theta) .
$$

\section{B. Hyperspherical adiabatic approach}

To solve Eq. (6) we can use a procedure known as the hyperspherical adiabatic approach with $R$ treated as an adiabatic parameter. A revision of the HAA can be found in Refs. [4-11]. The idea is to expand $\psi$ as follows:

$$
\psi(R, \Omega)=\sum_{\lambda} F_{\lambda}(R) \Phi_{\lambda}(R, \Omega)
$$


where the channel function $\Phi_{\lambda}$ satisfies the eigenvalue equation

$$
U(R, \Omega) \Phi_{\lambda}(R, \Omega)=U_{\lambda}(R) \Phi_{\lambda}(R, \Omega)
$$

for fixed $R$. The set of quantum numbers required to label the channel functions, here represented by $\lambda$, will be discussed in the next section. $F_{\lambda}(R)$ satisfies the equation

$\left\lfloor\frac{d^{2}}{d R^{2}}-\frac{U_{\lambda}(R)-\frac{1}{4}}{R^{2}}+\varepsilon_{\lambda}\right\rfloor F_{\lambda}(R)+\sum_{\lambda^{\prime}} W_{\lambda \lambda^{\prime}}(R) F_{\lambda^{\prime}}(R)=0$,

where the nonadiabatic terms are given by

$$
W_{\lambda \lambda^{\prime}}=2 P_{\lambda \lambda^{\prime}}(R) \frac{d}{d R}+Q_{\lambda \lambda^{\prime}}(R) \text {, }
$$

where

$$
\begin{aligned}
& P_{\lambda \lambda^{\prime}}(R)=\left\langle\Phi_{\lambda}\left|\frac{d}{d R}\right| \Phi_{\lambda^{\prime}}\right\rangle, \\
& Q_{\lambda \lambda^{\prime}}(R)=\left\langle\Phi_{\lambda}\left|\frac{d^{2}}{d R^{2}}\right| \Phi_{\lambda^{\prime}}\right\rangle .
\end{aligned}
$$

Differentiating $P_{\lambda \lambda^{\prime}}(R)$ with respect to $R$, we can relate $P_{\lambda \lambda^{\prime}}$ and $Q_{\lambda \lambda^{\prime}}$ by

$$
\frac{d P_{\lambda \lambda^{\prime}}}{d R}=Q_{\lambda \lambda^{\prime}}+R_{\lambda \lambda^{\prime}}
$$

where

$$
R_{\lambda \lambda^{\prime}}(R)=\left\langle\frac{d \Phi_{\lambda}}{d R} \mid \frac{d \Phi_{\lambda^{\prime}}}{d R}\right\rangle .
$$

If completeness is assumed $R_{\lambda \lambda^{\prime}}=P_{\lambda \lambda^{\prime}}^{2}$, and this furnishes a test for the completeness of the channel functionis. The solution of Eq. (10) will furnish the eigenenergies and the wave functions of our system.

\section{Adiabatic approximations and the basic inequalities}

The set of coupled equations (12) is still exact. If truncation is done, the result is the so-called coupled adiabatic approximation (CAA) [6]. Neglecting the coupling terms $W_{\lambda \lambda^{\prime}}(R), \lambda \neq \lambda^{\prime}$ in Eq. (12), the result is the uncoupled adiabatic approximation (UAA) [6]. In the UAA, the neglect of $W_{\lambda \lambda}(R)$ leads to the extreme adiabatic approximation (EAA) [6]. It can be proved [12] that the basic inequalities $\varepsilon_{\mathrm{EAA}} \leq \varepsilon \leq \varepsilon_{\mathrm{CAA}} \leq \varepsilon_{\mathrm{UAA}}$ hold for the ground-state energies. The EAA is a lower bound while UAA and CAA are upper bounds. An inequality similar to that exists for the extreme Born-Oppenheimer approximation (BOA) [12], namely, $\varepsilon_{\mathrm{BOA}} \leq \varepsilon$.

\section{POTENTIAL CURVES}

\section{A. Basis functions}

In order to solve Eq. (12) we first need to obtain the eigenpotentials $U_{\lambda}(R)$ from Eq. (11). We are interested in an efficient procedure to handle the numerical calculation such that the $U_{\lambda}(R)$ 's are precisely obtained for the whole range of $R$ and a wide range of masses of the three-body systems. In order to achieve it in an efficient way let us first look at the kinetic term of Eq. (7). That term has poles at $\alpha=0, \pi / 2$ which are independent of the masses of the system. On the other hand, the Coulomb interaction term, given by Eq. (8), has poles at $\alpha=0$ and at $(\theta, \alpha)=\left(\pi, \alpha_{+}\right)$and $\left(0, \alpha_{-}\right)$, where $\tan \alpha_{ \pm}=\eta_{ \pm}$. Thus the potential curves are obtained by considering regular solutions in the three regions $0 \leq \alpha \leq \alpha_{-}, \alpha_{-} \leq \alpha \leq \alpha_{+}$, and $\alpha_{+} \leq \alpha \leq \pi / 2$, and imposing continuity conditions at those boundary points.

In this kind of investigation it is important to select the appropriate angles and the corresponding harmonic functions as a basis for the problem. There exist different ways for selecting those angles. This was well studied by Smirnov and Shitikova [13]. Each possibility is identified with a possible chain in which the group $\mathrm{O}(6)$ can have its representation decomposed. We have chosen the chain

$$
\begin{array}{cccccc}
\mathrm{O}(6) & \supset \mathrm{O}(3) \times \mathrm{O}(3) \supset \mathrm{O}(3) \supset \mathrm{O}(2) \\
\downarrow & \downarrow & \downarrow & \downarrow & \downarrow \\
\left\{n l_{1} l_{2}\right\} & \left\{l_{1}\right\} & \left\{l_{2}\right\} & \{L\} & \{M\}
\end{array},
$$

which corresponds to the use of the tridimensional twoparticle spherical harmonics. The channel functions are then written as

$$
\begin{aligned}
\Phi_{\lambda}(R, \Omega)= & \sum_{l_{1}, l_{2}}(\sin \alpha)^{l_{1}+1}(\cos \alpha)^{l_{2}+1} \\
& \times \mathcal{Y}_{l_{1}, l_{2}}^{L_{M}}\left(\hat{\xi}_{1}, \hat{\xi}_{2}\right) g_{l_{1} l_{2}}^{\lambda}(R, \alpha),
\end{aligned}
$$

where $\mathcal{Y}_{l_{1} I_{2}}^{L M}$ is the usual two-particle coupled-orbital angular-momentum function $\left(\left|l_{1}-l_{2}\right| \leq L \leq l_{1}+l_{2}\right.$ and $\left.M=m_{1}+m_{2}\right)$. The basis functions $g_{l_{1} l_{2}}^{\lambda}(R, \alpha)$ at each $R$ are obtained from the solution of Eq. (11).

The motivation for the choice given by Eq. (18) instead of other possibilities (such as Euler angles [11] or the canonical form used in nuclear physics [8]) is the following. The decomposition into two $\mathrm{O}(3)$ groups preserves the individuality of the effective particles with respect to the angular motion in the $\left(\theta_{1}, \varphi_{1}\right),\left(\theta_{2}, \varphi_{2}\right)$ manifolds. However the introduction of the variables $\alpha$ and $R$ will account for the collective effects

We note that the function $g_{l_{1} l_{2}}(R, \alpha)$ in Eq. (18) was not further expanded in any basis and will be calculated by a new method presented in this paper. In this point our approach differs from the usual techniques which are based on some kind of expansion using orthonormal polynomials [4-6,8-11] or another [11] set of functions with faster convergence properties.

The use of any kind of polynomial set assumes the separability of the variables $\alpha$ and $R$, i.e.,

$$
\Phi_{\lambda}(R, \Omega)=\sum_{n, l_{1}, l_{2}} \chi_{n l_{1} l_{2}}^{\lambda}(R) Y_{n l_{1} l_{2}}^{L M}(\Omega)
$$

where the complete orthonormal set $\left\{\mathscr{Y}_{n I_{1} I_{2}}^{L M}\right\}$ are hyperspherical harmonics [2]. The hyperspherical-harmonic approach corresponds to the prescription 
$\mathcal{S}_{l_{1} l_{2}}^{\lambda}(R, \alpha)=\sum_{n} \chi_{n l_{1} l_{2}}^{\lambda}(R) P_{n}^{l_{1}+1 / 2, l_{2}+1 / 2}(\cos (2 \alpha))$

where $P_{n}^{l_{1}+1 / 2, l_{2}+1 / 2}$ is the usual Jacobi polynomial. Thus the solution of Eq. (11) using (20) reduces the prob. lem to a matrix diagonalization $[6,8]$. This way, although quite straightforward, has its limitations. For instance, a known limitation is due to the slow convergence because asymptotic solutions are not easily expanded in terms of $Y_{n l_{1} l_{2}}^{L M}(\Omega)$. This problem becomes very severe for longrange forces. However for short-range forces, such as the ones encountered in nuclear physics, this procedure is quite successful [8]. To overcome this problem in Coulombic three-body systems, Lin and Liu [11] considered a new basis function set but it has the disadvan. tage of not being an orthonormal set. In addition numer. ical difficulties are not completely removed for large- $R$ values. The situation is in some sense dramatic if we no. tice that for a truncated calculation, using Jacobi polynomials, potential curves necessarily appear with wrong behavior for large $R$. In order to avoid these problems we shall solve directly the infinite set of differential equations obtained by substituting Eq. (18) into Eq. (11), namely,

$$
\left\{\frac{d^{2}}{d \alpha^{2}}+2\left[\left(l_{1}+1\right) \cot \alpha-\left(l_{2}+1\right) \tan \alpha\right] \frac{d}{d \alpha}-U_{\lambda}(R)-\left(l_{1}+l_{2}+2\right)^{2}\right\} g_{l_{1} l_{2}}^{\lambda}(R, \alpha)-R \sum_{l_{1}^{\prime} l_{2}^{\prime}} \widetilde{v}_{l_{1} l_{2} l_{1}^{\prime} l_{2}^{\prime}}^{L M}(\alpha) S_{l_{1}^{\prime} l_{2}^{\prime}}^{\lambda}(R, \alpha)=0,
$$

where

$$
\begin{aligned}
\delta_{l_{1} l_{2} l_{1}^{\prime} l_{2}^{\prime}}^{L M}(\alpha)= & (\sin \alpha)^{l_{1}^{\prime}-l_{1}}(\cos \alpha)^{l_{2}^{\prime}-l_{2}} \\
& \times\left\langle\mathcal{Y}_{l_{1} l_{2}}^{L M}|\hat{v}| y_{l_{1}^{\prime} l_{2}^{\prime}}^{L M}\right\rangle .
\end{aligned}
$$

An explicit expression for $\langle\hat{v}\rangle$ is given in the Appendix. To solve Eq. (21) we should be aware of the symmetries imposed in the hyperspherical-harmonic functions $S_{l_{1} l_{2}}^{\lambda}(R, \alpha)$. A discussion of this point can be seen for instance in Refs. [7] and [11].

\section{B. Small- and large- $R$ behaviors}

It is remarkable that Eq. (21) admits exact solutions in the limit $R=0$. For instance, in that limit and using a new variable $\alpha=\alpha^{\prime} / 2$ and the transformation

$$
\widetilde{\mathfrak{S}}_{l_{1} l_{2}}^{\lambda}(R, \alpha)=(\sin \alpha)^{l_{1}+1}(\cos \alpha)^{l_{2}+1} g_{l_{1} l_{2}}^{\lambda}(R, \alpha),
$$

we obtain

$$
\begin{aligned}
& \left\lfloor-\frac{d^{2}}{d \alpha^{\prime 2}}+\frac{4 l_{1}\left(l_{1}+1\right)}{16 \sin ^{2}\left(\alpha^{\prime} / 2\right)}+\frac{4 l_{2}\left(l_{2}+1\right)}{16 \cos ^{2}\left(\alpha^{\prime} / 2\right)}\right. \\
& \left.+\frac{1}{4} U_{\lambda}(0)\right] S_{l_{1} l_{2}}^{\lambda}(0, \alpha)=0,
\end{aligned}
$$

which compared with the canonical form of the Jacobi equation [14]

$$
\begin{gathered}
{\left[-\frac{d^{2}}{d \alpha^{\prime 2}}-\frac{1-4 a^{2}}{16 \sin ^{2}\left(\alpha^{\prime} / 2\right)}-\frac{1-4 b^{2}}{16 \cos ^{2}\left(\alpha^{\prime} / 2\right)}\right.} \\
\left.-\left[\lambda+\frac{a+b+1}{2}\right]^{2}\right] Q_{\lambda}^{(a, b)}\left(\cos \alpha^{\prime}\right)=0
\end{gathered}
$$

has the regular solution

$$
\begin{aligned}
Q_{\lambda}^{(a, b)}\left(\cos \alpha^{\prime}\right)= & {\left[\sin \left(\alpha^{\prime} / 2\right)\right]^{a+1 / 2}\left[\cos \left(\alpha^{\prime} / 2\right)\right]^{b+1 / 2} } \\
& \times P_{\lambda}^{(a, b)}\left(\cos \alpha^{\prime}\right),
\end{aligned}
$$

where $P_{\lambda}^{(a, b)}$ are Jacobi polynomials.
Therefore the potential curves at $R=0$ have the form

$$
U_{\lambda}(0)=\left(2 n+j_{1}+j_{2}+2\right)^{2},
$$

and the $\widetilde{\mathscr{S}}$ functions are given by

$$
\begin{aligned}
\widetilde{g}_{n j_{1} j_{2}}^{L M}(0, \alpha)= & (\sin \alpha)^{l_{1}+1}(\cos \alpha)^{l_{2}+1} \\
& \times P_{n}^{l_{1}+1 / 2, l_{2}+1 / 2}(\cos (2 \alpha)) \delta_{l_{1} j_{1}} \delta_{l_{2} j_{2}} .
\end{aligned}
$$

Since there are no spin interactions, the spatial wave function has well-defined quantum numbers, $L, M$, spin $S$, and parity. At $R=0$, we see explicitly from Eqs. (26) and (27) that the potential curves are labeled by $\lambda=\left(n, j_{1}, j_{2}\right)$. For notational simplicity we have omitted the conserved quantum numbers, $L, M, S$, and parity. The allowed values of $j_{1}$ and $j_{2}$ are dictated by the triangular relation with $L$ and the required symmetry of the problem imposed by the parity. There is no restriction on the integer values of $n$. If the nonadiabatic couplings $W_{\lambda \lambda^{\prime}}(R)$ [see Eq. (12)] are neglected, $\lambda=\left(n, j_{1}, j_{2}\right)$ will be conserved.

The inclination of the curve $U_{\lambda}(R)$ at $R=0$ is obtained by calculating the derivative of Eq. (11) and using Eq. (9). As a result, we get

$$
\begin{aligned}
\hat{v}(\alpha, \theta) \Phi_{\lambda}(0, \Omega)+\left[U(0)-U_{\lambda}(0)\right]\left(\Phi_{\lambda}^{\prime}\right)_{R}=0 \\
=\left(U_{\lambda}^{\prime}\right)_{R}=0 \Phi_{\lambda}(0, \Omega) .
\end{aligned}
$$

The prime symbol on $\Phi_{\lambda}$ and $U_{\lambda}$ indicates the derivative with respect to $R$ of those quantities. Multiplying Eq. (28) by $\Phi_{\lambda^{*}}^{*}(0, \Omega)$ and integrating over $d \Omega$ we obtain

$$
\begin{aligned}
\left\langle\Phi_{\lambda^{\prime}}(R, \Omega)|\hat{v}(\alpha, \theta)| \Phi_{\lambda}(R, \Omega)\right\rangle_{R=0} & \\
+\left\langle\Phi_{\lambda^{\prime}}(R, \Omega)\left|\left[U(0)-U_{\lambda}(R)\right]\right|\right. & \left|\Phi_{\lambda}^{\prime}(R, \Omega)\right\rangle_{R=0} \\
= & \left(U_{\lambda}^{\prime}\right)_{R=0} \delta_{\lambda^{\prime} \lambda} .
\end{aligned}
$$

For $\lambda^{\prime}=\lambda$,

$$
\left(U_{\lambda}^{\prime}\right)_{R=0}=\left\langle\Phi_{\lambda}|\hat{v}(\alpha, \theta)| \Phi_{\lambda}\right\rangle_{R=0},
$$

where $\lambda=\left(n, j_{1}, j_{2}\right)$.

For $\lambda^{\prime} \neq \lambda$, using Eq. (14), we have 
$\left(\Phi_{\lambda^{\prime}}|\hat{U}(\alpha, \theta)| \Phi_{\lambda}\right\rangle_{R=0}=-\left(U_{\lambda^{\prime}}-U_{\lambda}\right)_{R=0} P_{\lambda^{\prime} \lambda}(0)$

or equivalently,

$P_{\lambda^{\prime} \lambda}(0)=-\left\langle\Phi_{\lambda^{\prime}}|\hat{v}(\alpha, \theta)| \Phi_{\lambda}\right\rangle_{R=0} /\left(U_{\lambda^{\prime}}-U_{\lambda}\right)_{R=0}$.

The analysis for large $R$ is discussed in Refs. $[4,7,11]$.

\section{Set of differential equations with rational coefficients}

Although solutions for Eq. (21) in the variable $\alpha$ (for fixed $R$ ) exist, the form of that equation is not appropriate for the use of the Cauchy theorem. The reason lies in the nonpolynomial structure of the trigonometric functions which appear there and the intractability of the series expansions of such functions. Thus we have searched for a new variable which can transform Eq. (21) into a form whose coefficients are rational functions. Therefore analytical solutions can be obtained in the usual power-series expansion. That variable is $z=\tan (\alpha / 2)$. As a result, the expansion coefficients given by recursion formulas can be calculated numerically for fixed values of $R$. In the variable z, Eq. (21) reads

$$
\begin{aligned}
& \left\lfloor\frac{d^{2}}{d z^{2}}+\mathcal{P}_{l_{1} l_{2}}(z) \frac{d}{d z}+Q_{l_{1} l_{2}}(R, z)\right\rfloor g_{l_{1} l_{2}}^{\lambda}(R, z) \\
& \quad+R \sum_{l_{1}^{\prime}, l_{2}^{\prime}} R_{l_{1} l_{1}^{\prime} l_{2} l_{2}^{\prime}}(z) \mathcal{S}_{l_{1}^{\prime} l_{2}^{\prime}}^{\lambda}(R, z)=0,
\end{aligned}
$$

where

$$
P_{l_{1} l_{2}}(z)=\frac{2}{1+z^{2}}\left[z+\frac{\left(l_{1}+1\right)\left(1-z^{2}\right)}{z}-\frac{4\left(l_{2}+1\right) z}{1-z^{2}}\right],
$$

$$
\begin{aligned}
Q_{l_{1} l_{2}}(R, z)= & -4\left[\left(l_{1}+l_{2}+2\right)^{2}+U_{\lambda}(R)\right] /\left(1+z^{2}\right)^{2} \\
& -\left(\frac{\mu_{1}}{\mu}\right]^{1 / 2} q_{1} q_{2} \frac{2}{z\left(1+z^{2}\right)}
\end{aligned}
$$

and

$$
\begin{aligned}
\mathcal{R}_{l_{1} l_{1}^{\prime} l_{2} l_{2}^{\prime}(z)=-} & \frac{4}{\left(1+z^{2}\right)^{2}}\left(\frac{2 z}{1+z^{2}}\right)^{l_{1}^{\prime}-l_{1}}\left(\frac{1-z^{2}}{1+z^{2}}\right)^{l_{2}^{\prime}-l_{2}} \\
\times & {\left[\frac{m_{1}}{\sqrt{\mu \mu_{1}}} q_{1} q_{3} \Delta_{l_{1} l_{1}^{\prime} l_{2} l_{2}^{\prime}(z)}^{+}\right.} \\
& \left.+\frac{m_{2}}{\sqrt{\mu \mu_{2}}} q_{2} q_{3} \Delta_{l_{1} l_{1}^{\prime} l_{2} l_{2}^{\prime}}^{-}(z)\right]
\end{aligned}
$$

The expressions for $\Delta^{+}$and $\Delta^{-}$are given in the AppenThe singular points of Eq. (8) occur at $\mathrm{n}\left(\alpha_{ \pm} / 2\right)$. The singular points of $\mathrm{Eq}$. (33) are $\pm 1, \pm i$. The singular points are drawn in the plex-z plane shown in Fig. 1. As $0 \leq \alpha \leq \pi / 2$, it is imthat $0 \leq z \leq 1$. Thus only the region $\operatorname{Re}(z) \geq 0$ is of sical interest in the solution of Eq. (33). That equaa can be solved by a local power-series method for the ction $g_{l_{1} l_{2}}^{\lambda}(R, z)$ in each interval $\left[0, z_{-}\right],\left[z_{-}, z_{+}\right]$, and $\left.z_{+}, 1\right]$. Its solution has an exponential behavior [7] for

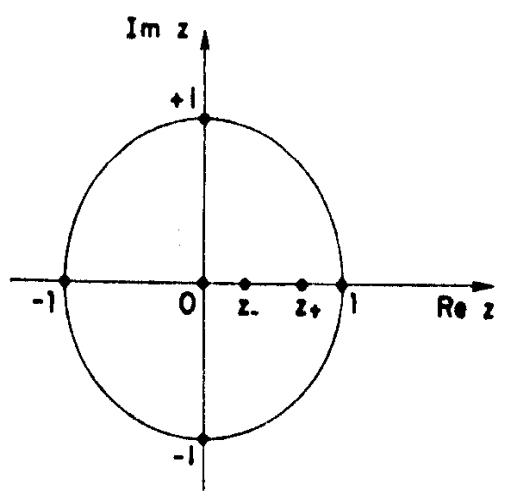
(33).

FIG. 1. Complex-z plane showing the singular points of Eq.

large $R$, which can be built in. The resulting series is therefore rapidly convergent. Another aspect of Eq. (33) is its behavior at small $R$. In order to get a polynomial form at $R=0$, we should introduce [7] a factor $\left(1+z^{2}\right)^{s}$, where $s=l_{1}+l_{2}+2-\sqrt{U_{\lambda}(0)}$, and $U_{\lambda}(0)$ is given by Eq. (26). That series-expansion procedure generates an algebraic set of recursion relations for the series coefficients. To obtain the recursion relations from Eq. (33) is straightforward but tedious, so a computer code was written to extract it algebraically without performing floating-point operations. The same code also generates a FORTRAN program to solve the relations on a $\mu \mathrm{VAX}$ computer. One of the advantages of this method is to isolate two types of error. One comes from the truncation of the solution of Eq. (33) through the series-convergence procedure for a maximum number $N$ of terms, and the other appears in the successive inclusion of angularmomentum channels until a limit value $l_{\max }$. Thus the coefficients are numerically calculated in an exact way. That can be verified afterward using quadruple precision floating-point arithmetics. The procedure of calculation, once the truncation limits are stated, begins with a tentative value for the potential $U_{\lambda}(R)$ at a point $R$, selected in practice by the application of the Newton forwarddifference method [14] to the previously calculated points. Once a good tentative point is selected, the recursion relations are solved for arbitrary initial conditions in the regions $\left[0, z_{-}\right]$and $\left[z_{+}, 1\right]$. The singular behavior of the differential equation in those points are then exactly incorporated in the algorithm without causing any error or instability. Finally the equations are solved in the in. termediate region by imposing continuity conditions which will simultaneously select the regular combination of the already calculated independent solutions and the potential curve. The determinantal condition which emerges from the procedure described above is used for the update of the value of $U_{\lambda}(R)$ and the process continues until a desired precision (machine precision) is reached. The same strategy is then repeated for the next value of $R$. As a result we have controlled truncation errors. The fundamental property of the chosen variable $z$ is that the differential equation can undergo an arbitrary translation transformation without losing its polynomial character. This fact allows us to select smaller grids in the $z$ space different from the natural ones of the three regions. Hence the method described above can also be 
combined with finite-difference techniques without losing its "exact" structure. This will be particularly important for systems with very different masses.

The singularities of Eq. (33) are shown in Fig. 1. The poles at \pm 1 and $\pm i$ define a convergence circle of unity radius in which a regular solution at $z=0$ exists. Depending on the constituent masses of a given Coulombic system, the singularities $z_{+}$and $z_{-}$will move along $\operatorname{Re}(z)$ defining two inner circles, in which Eq. (33) must be solved. Pole locations for several systems are shown in Table 1. We can see that for systems with large mass ratio $\left(m_{2} / m_{3} \gg 1\right)$, like $d_{2}{ }^{+}$, singularities will be pushed to the border limit, causing the known "large-mass" problem [9] in the calculation of the potential curves.

We should stress that machine precision in the truncation procedure was obtained with typical $N$ values between 50 and 200 depending on the system, allowing us to carefully analyze the convergence trends in the angularmomentum expansion.

The solution of Eq. (33) will then give $U_{\lambda}(R)$ in a precise way. With this information at hand we can finally solve Eq. (12), by standard procedures, to obtain the energies and wave functions of the problem.

\section{APPLICATIONS}

We give and discuss below the numerical results for several carefully chosen physical systems. This choice was mainly concerned with the topological locations of $\left(z_{-}, z_{+}\right)$when compared with the border singularities $(0,1)$.

\section{A. He}

In order to appraise the accuracy of our approach, we present in Fig. 2 the potential curve for the helium ground state, calculated by our method and by matrix diagonalization. In the second method, the channel functions are expanded in $16,20,25$, and $40 \mathrm{Jacobi}$ polynomials, respectively. We can see that matrix diagonalization furnishes slow convergence even in the intermediate range of $R$. In addition, the asymptotic values of $U_{0}(R) / R^{2}$ are not correct, i.e., the truncated curves cross the ionization limit, resulting in unphysical results at large $R$.

In Table II we give the values of $U_{0}(R)$ at its minimum as a function of $l_{\max }=1, \ldots, 30$, and $N=70,90,130$. The

TABLE I. Pole locations for several physical systems. Only $\left(\alpha_{-}, \alpha_{+}\right)$and $\left(z_{-}, z_{+}\right)$are listed.

\begin{tabular}{ccccc}
\hline \hline System & $\alpha_{-}$ & $\alpha_{+}$ & $z_{-}$ & $z_{+}$ \\
\hline $\mathrm{He}$ & $\pi / 4$ & $\pi / 2$ & 0.41421 & 1 \\
$d d_{\mu}$ & 1.40677 & $\alpha_{-}$ & 0.84809 & $z_{-}$ \\
$d_{2}{ }^{+}$ & 1.55913 & $\alpha_{-}$ & 0.98840 & $z_{+}$ \\
$\mathrm{InSb}$ & 1.31751 & $\pi / 2$ & 0.77411 & 1 \\
$\mathrm{ZnSe}$ & 1.18285 & $\pi / 2$ & 0.67162 & 1 \\
$\mathrm{CdS}$ & 1.15026 & $\pi / 2$ & 0.64823 & 1 \\
$\mathrm{ZnO}$ & 1.14110 & $\pi / 2$ & 0.64174 & 1 \\
$\mathrm{CdSe}$ & 1.13222 & $\pi / 2$ & 0.63549 & 1 \\
$p d \mu$ & 1.437 & 1.308 & 0.874 & 0.767 \\
\hline \hline
\end{tabular}

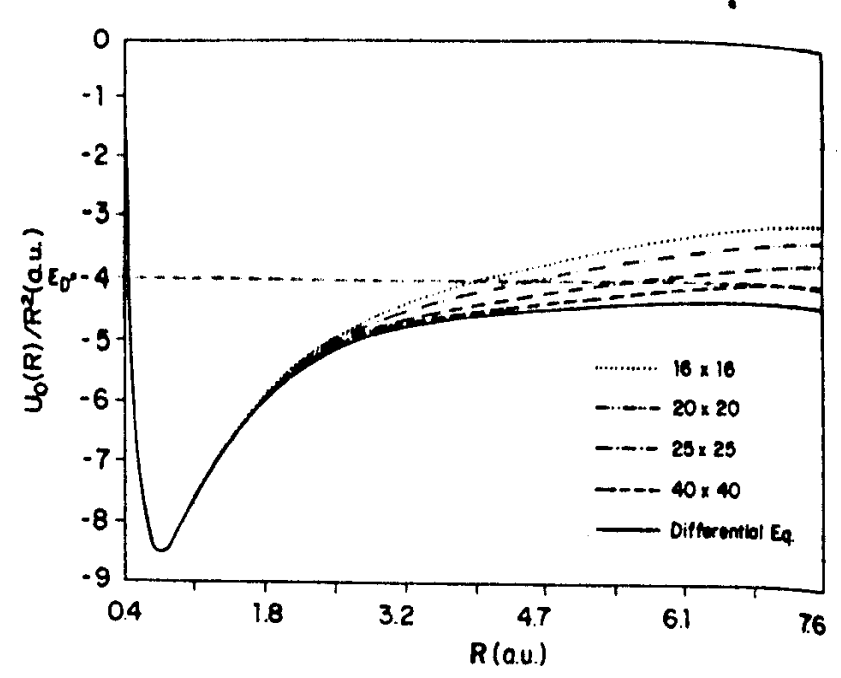

FIG. 2. Convergence trends of the solutions obtained by the diagonization procedure and by the solution of the angular differential equations for the $\mathrm{He}$ atom. The second method gives precise convergence. For each curve, obtained by the diagonization procedure, matrix dimensionality is given.

agreement between $N=90$ and 130 is of a few parts in $10^{8}$. No difference can be observed in the potential curves if a larger value of $N$ is taken. Numerical errors have been searched for using REAL* 16 precision and no difference was observed. The exponential factor mentioned in the preceding section causes a regular and stable behavior at large $R$ even with a small number of terms in the series expansion. The angular-momentum convergence up to 30 channels is also shown in Table II. The convergence is much slower and we expect corrections of few parts in $10^{8}$ if more angular-momentum channels are considered. The computation time is roughly proportional to $\mathrm{Nl}_{\max }^{2}$.

\section{B. Excitons}

The behavior of excitons trapped by ions in semiconductors can be treated as the motion of dressed electrons and holes under the influence of the ion field in a medium of dielectric constant $\varepsilon$. Changing the semiconductor material, we can create different Coulombic systems with distinct mass ratios $m_{2} / m_{3}$. In Table III we list the properties of several semiconductors. In Fig. 3, we plot $U_{0}(R) / R^{2}$ for several semiconductor materials. The

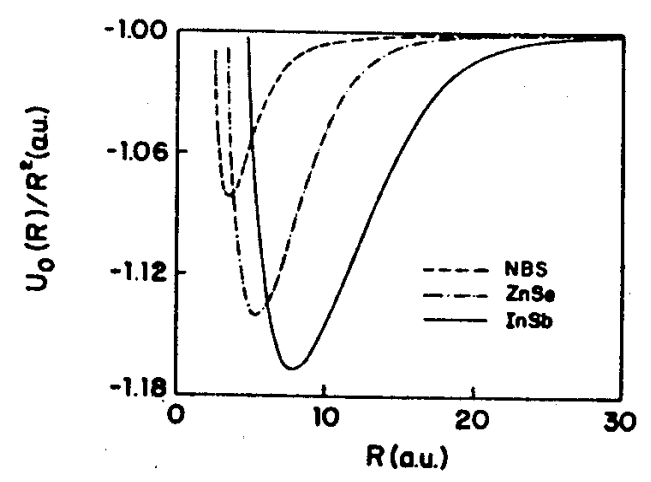

FIG. 3. Plot of $U_{0}(R) / R^{2}$ vs $R$ for several semiconductor materials. The material "crit" is defined in the text. Units are in $\hbar=e^{2} / \varepsilon=m_{e}=1$. 
TABLE II. Calculated values of $U_{0}(R)$ (in $\mathrm{eV}$ ) at its minimum as a function of $N$ (the maximum number of terms of the power-series expansion) and $l_{\max }$ (maximum value of the angular momentum $\left.l_{1}=l_{2}(L=0)\right]$ for He.

\begin{tabular}{|c|c|c|c|}
\hline$N$ & 70 & 90 & 130 \\
\hline 2 & -8.48231553211019 & -8.48231553211019 & -8.482315532110192 \\
\hline 3 & -8.48920864599310 & -8.48920864599310 & -8.489208645993092 \\
\hline 4 & -8.49095792163346 & -8.49095792163346 & -8.490957921633461 \\
\hline 5 & -8.49159146281478 & -8.49159146281478 & -8.491591462814778 \\
\hline 6 & -8.49187363748107 & -8.49187363748107 & -8.491873637481055 \\
\hline 7 & -8.49201766919034 & -8.49201766919017 & -8.492017669190149 \\
\hline 8 & -8.49209866347905 & -8.49209866347691 & -8.492098663476909 \\
\hline 9 & -8.49214762996351 & -8.49214762994565 & -8.492147629945638 \\
\hline 10 & -8.49217894537702 & -8.49217894526849 & -8.492178945268362 \\
\hline 11 & -8.49219989265177 & -8.49219989220362 & -8.492199892202962 \\
\hline 12 & -8.49221442887031 & -8.49221442762526 & -8.492214427621516 \\
\hline 13 & -8.49222482952850 & -8.49222482709776 & -8.492224827078434 \\
\hline 14 & -8.49223246596052 & -8.49223246235439 & -8.492232462276760 \\
\hline 15 & -8.49223819843634 & -8.49223819373397 & -8.492238193504505 \\
\hline 16 & -8.49224258666084 & -8.49224257938334 & -8.492242578877736 \\
\hline 17 & -8.49224600889160 & -8.49224599208467 & -8.492245991220818 \\
\hline 18 & -8.49224873364987 & -8.49224868722191 & -8.492248686020558 \\
\hline 19 & -8.49225096455925 & -8.49225084376133 & -8.492250842335194 \\
\hline 20 & -8.49225286536512 & -8.49225258971338 & -9.492252588186838 \\
\hline 21 & -8.49225456546763 & -8.49225401843237 & -8.492254016755217 \\
\hline 22 & -8.49225614809178 & -8.49225519948088 & -8.492255196956057 \\
\hline 23 & -8.49225763318729 & -8.49225618630452 & -8.492256180500316 \\
\hline 24 & -8.49225897601781 & -8.49225702202973 & -8.492257006698077 \\
\hline 25 & -8.49226009609408 & -8.49225774390441 & -8.492257705790296 \\
\hline 26 & -8.49226092750891 & -8.49225838606801 & -8.492258301298996 \\
\hline 27 & -8.49226146016671 & -8.49225897970837 & -8.492258811709149 \\
\hline 28 & -9.49226174528647 & -8.49225954987691 & -8.492259251683967 \\
\hline 29 & -8.49226186650240 & -8.49226010979849 & -8.492259632955843 \\
\hline 30 & -8.49226190234978 & -8.49226065585292 & -8.492259964987300 \\
\hline
\end{tabular}

word "crit" [17] appearing in Fig. 3 stands for a fictitious material for which there is no bound state. In the last two columns of Table III we show the calculated ionization energies in the EAA. We compare them with variational calculations done by other authors. The bound states of those systems are very close to the ionization threshold. They get closer to it as the mass ratio $m=m_{h}^{*} / m_{e}^{*}$ decreases. Notice that $m_{e}^{*}$ and $m_{h}^{*}$ are the dressed masses of the electron and the hole, respectively. The calculated energies, as we have proved elsewhere [12], should satisfy the basic inequalities. This is verified in Table III with the exception of InSb. In this case the considered number of angular-momentum channels was not large enough to achieve energy convergence. On the other hand a precise calculation concerning this problem was done for $\mathrm{ZnSe}$ where up to ten angular-momentum channels $(l=0,1,2, \ldots, 9)$ were considered in order to achieve convergence. The results for $\mathrm{ZnSe}$ are shown in Fig. 4. In Table IV the convergence is also studied for $\mathrm{ZnSe}$ for $N=80,100,120$ and $l$ up to 9 . As we expect, the convergence is in general slowed when the mass ratio departs from unity and the singularities approach the convergence limit. We can see that a relatively large number of channels should be considered in order to

TABLE III. Binding energies for several semiconductors. $E_{D}$ is the dissociation energy. The superscripts refer to variational calculations. Notice that $\varepsilon$ is the static dielectric constant of the material and the values are taken from Ref. [15].

\begin{tabular}{|c|c|c|c|c|c|c|c|c|c|}
\hline Material & & $m_{e}^{*}(\mathrm{amu})$ & $m_{h}^{*}$ (amu) & $m_{e}^{*} / m_{h}^{*}$ & $E_{D}(\mathrm{meV})$ & \multicolumn{2}{|c|}{$E-E_{D}(\mathrm{meV})$} & \multicolumn{2}{|c|}{$\left(E-E_{D}\right) / E_{D}(\%)$} \\
\hline InSb & 16.80 & 0.02 & 0.30 & 0.067 & 0.9641 & 0.0893 & $0.0993^{\star}$ & 9.26 & $10.30^{n}$ \\
\hline $\mathrm{ZnSe}$ & 9.10 & 0.10 & 0.60 & 0.167 & 16.43 & 0.751 & $0.676^{\circ}$ & 4.57 & $4.12^{\prime \prime}$ \\
\hline CdS & 10.33 & 0.20 & 1.00 & 0.20 & 25.50 & 1.161 & $1.0^{b}$ & 3.60 & $3.10^{b}$ \\
\hline $\mathrm{ZnO}$ & 8.50 & 0.24 & 1.14 & 0.21 & 45.19 & 2.357 & $1.9^{b}$ & 3.35 & $2.70^{b}$ \\
\hline CdSe & 10.66 & 0.13 & 0.59 & 0.22 & 15.56 & 0.486 & $0.348^{\circ}$ & 3.12 & $2.23^{\circ}$ \\
\hline
\end{tabular}

See Ref. [15].

'See Ref. [16]. 
TABLE IV. The same as Table II for $\mathrm{ZnSe}$, but energies are in meV.

\begin{tabular}{cccc}
\hline \hline$l_{\max }$ & 80 & 100 & 120 \\
\hline 3 & -18.28664525363347 & -18.28664532411643 & -18.28664532493239 \\
4 & -18.49081944068719 & -18.49081992721156 & -18.49081995106005 \\
5 & -18.61172684274993 & -18.61172541504315 & -18.61172566280509 \\
6 & -18.68610842091153 & -18.68608452757457 & -18.68608562348481 \\
7 & -18.73371095301742 & -18.73360063611852 & -18.73360257476791 \\
8 & -18.76539459421911 & -18.76507912451675 & -18.76507693102769 \\
9 & -18.78731472430117 & -18.78663596187230 & -18.78661326029223 \\
\hline
\end{tabular}

reach convergence. With our results, the variational calculations $[15,16]$, and the knowledge of the basic inequalities [12], we can establish lower- and upper-bound relations for those systems. However one should have in mind that the lower-bound character of the EAA will only be granted if convergence in the angular momentum is achieved.

\section{C. $d d \mu$ and $d_{2}+$}

Muon catalyzed fusion [18-20] has in part motivated us in the calculation of the potential curves of the quasilinear $d d \mu$ molecule. Jacobi coordinates $\left(\xi_{1}, \xi_{2}\right)$ are chosen such that one of them, namely, $\xi_{1}$, is taken as the nuclear separation (deuteron-deuteron distance). With the use of this "Born-Oppenheimer"-type approximation, the potential curve goes to zero as $R$ gets large as it should. The convergence trend of the potential curve is shown in Fig. 5. The graphs are for 1-5 channels. In this case, due again to different masses, the convergence is achieved only with a precision of $10 \mathrm{eV}$. The consideration of $d d \mu$ and $d_{2}{ }^{+}$in this work is due to the fact that they are the ones most troublesome to analyze within the HAA. Therefore this is a crucial test for the

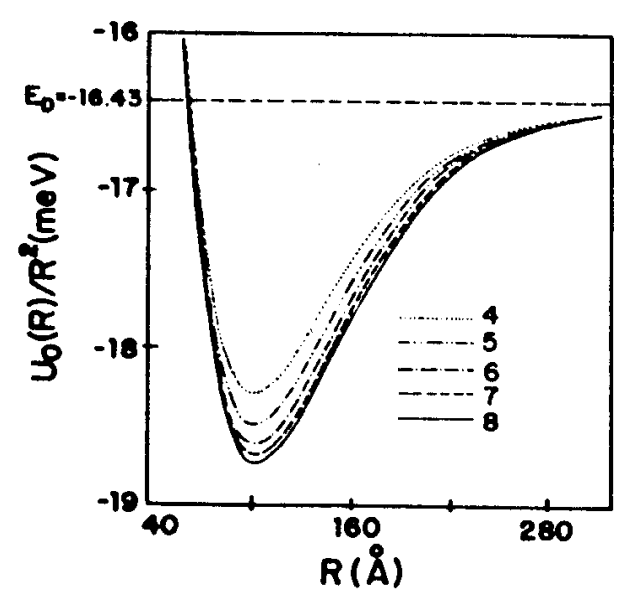

FIG. 4. Potential curves as a function of the number of angular channels for $\mathrm{ZnSe}$. Graphs here are given for 4-8 channels. Note that convergence requires the use of a large number of channels in the calculation. The deeper the curve the larger the number of channels required. use of the present techniques. The $d d \mu$ system can only be handled by the present approach if the finite-difference technique mentioned in the last section is used. In Table $\mathrm{V}$ we give the values of $U_{0}(R) / R^{2}$ at its minimum as a function of $N$ and $l_{\max }$.

As a final example we give the preliminary results for the $d_{2}{ }^{+}$molecule. The potential curve for $U_{0}(R) / R^{2}$ is shown in Fig. 6. We have chosen the system $d_{2}{ }^{+}$because it illustrates well the topology of the problem. Its singularity $\left(z_{-}=z_{+}\right)$is very close to $z=1$ (see Fig. 1), causing slow convergence in the series, requiring therefore more computer CPU time. The inclusion of more channels should improve the results, as it happened for the $d d \mu$ system. We are presently working on this calculation.

\section{v. CONCLUSIONS}

We present in this paper a general method of handling the troublesome Coulombic three-body systems through the hyperspherical adiabatic approach. For long-range forces no matter what method is chosen we might face convergence problems in the determination of the solutions. This may not be different even if the exact HAA is

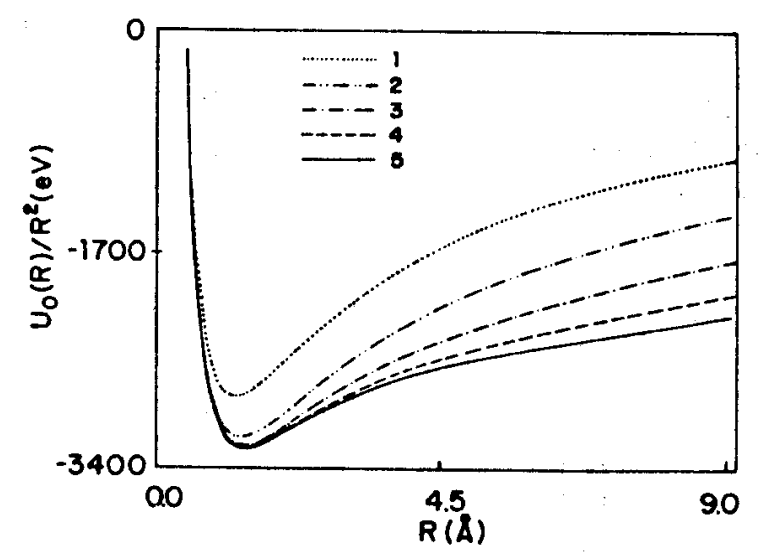

FIG. 5. Potential curves as a function of the number of angular channels for $d d \mu$. Graphs here are given for $1-5$ channels. Note that convergence requires the use of a large number of channels in the calculation. The deeper the curve the larger the number of channels required. The abscissa is the hyperspherical radius multiplied by $\left(2 m_{e} / m_{D}\right)^{1 / 2}$ in order to establish a correspondence with the internuclear separation [see Eq. (1)]. $R$ is in units of $\hbar^{2} / m_{\mu} e^{2}$. 
TABLE V. Calculated values of $U_{0}(R) / R^{2}$ at its minimum as a function of $N$ (the maximum number of terms of the power-series expansion) and $l_{\max }$ [maximum value of the angular momentum $\left.l_{1}=l_{2}(L=0)\right]$ for $d d \mu$.

\begin{tabular}{lcccc}
\hline \hline$N$ & 150 & 250 & 350 & 450 \\
\hline$l_{\max }$ & & & & \\
\hline 1 & 2816.118270341 & 2816.118270612 & 2816.118270612 & 2816.118270612 \\
2 & 3154.341168834 & 3154.340757821 & 3154.340757821 & 3154.340757821 \\
3 & 3219.416388365 & 3219.397817965 & 3219.397819028 & 3219.397819024 \\
4 & 3237.506651807 & 3237.413893992 & 3237.413849613 & 3237.413846365 \\
5 & 3244.219681145 & 3244.050239226 & 3244.049625397 & 3244.049383539 \\
\hline \hline
\end{tabular}

used. However by using a suitable variable, namely, $z=\tan (\alpha / 2)$, Eq. (11) can be transformed into the important Eq. (33), which can be solved by a power-series expansion, giving rise to a stable and converging solution valid for small and large values of the hyperspherical radius $R$. The solution of Eq. (33) furnishes simultaneously and in a precise way $U_{\lambda}(R)$, which is basically the ultimate physical goal in the calculation of the Coulombic three-body systems. Analysis of the mathematical singularities of Eq. (33) offers an insight into the physics of the problem and in the determination of stable converging solutions as well.

The approach presented here is exact, theoretically transparent, and general enough to be used successfully in the description of three-body systems. Although developed here for long-range forces, it can be applied to intermediate- and short-range forces as well. This version of the HAA permits the exact calculation of potential curves, binding energies, resonances, and possibly scattering states. In order to illustrate our approach we have considered several Coulombic three-body systems: $\mathrm{He}, d d \mu, d_{2}{ }^{+}$, and excitons bound to a Coulomb center in different semiconductors.

The theoretical description of Coulombic three-body systems is no longer hampered by lack of an efficient

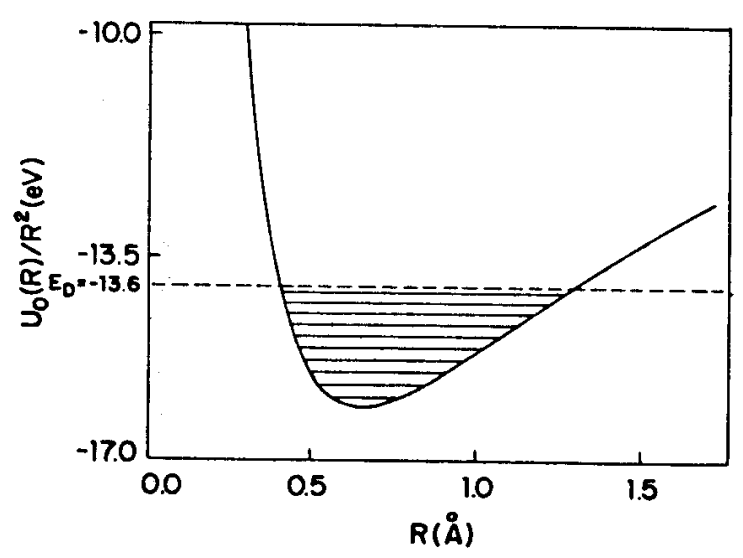

FIG. 6. Plot of $U_{0}(R) / R^{2}$ vs $R$ for $d_{2}{ }^{+}$. Horizontal lines represent some of the lowest bound states supported by $U_{0}(R)$. They are below the dissociation energy $E_{D}=-13.605 \mathrm{eV}$. The abscissa is the hyperspherical radius multiplied by $\left(2 m_{e} / m_{D}\right)^{1 / 2}$ in order to establish a correspondence with the internuclear separation [see Eq. (1)]. $R$ is in units of $\hbar^{2} / m_{\mu} e^{2}$. method for calculating potential curves and channel functions. The approach presented in this work, in our view, will prove to be useful.

\section{ACKNOWLEDGMENTS}

This work was partially supported by Conselho $\mathrm{Na}$ cional de Desenvolvimento Cientifico e Tecnológico $(\mathrm{CNPq})$, Financiadora de Estudos e Projetos (FINEP), Fundação de Amparo à Pesquisa de Săo Paulo (FAPESP), and Fundação de Amparo à Ciência e Tecnologia de Pernambuco (FACEPE) (Brazilian agencies).

\section{APPENDIX: EVALUATION OF THE CHANNEL MIXING COEFFICIENTS}

To calculate the matrix elements of $v$ in Eq. (22), because of the form of Eq. (8), we first need to evaluate the tensor

$$
\begin{aligned}
T_{l_{1} l_{1}^{\prime} l_{2}^{\prime} l_{2}^{\prime}}^{L M \pm}(\alpha)=\int d \hat{\xi}_{1} d \hat{\xi}_{2}\left[Y_{l_{1}^{\prime} l_{2}^{\prime}}^{L M}\left(\hat{\xi}_{1}, \hat{\xi}_{2}\right)\right]^{*} \\
\quad \times\left(\sin ^{2} \alpha+\eta_{ \pm}^{2} \cos ^{2} \alpha\right. \\
\left.\quad \pm \eta_{ \pm} \sin 2 \alpha \cos \theta\right)^{-1 / 2} Y_{l_{1} l_{2}}^{L M}\left(\hat{\xi}_{1}, \hat{\xi}_{2}\right)
\end{aligned}
$$

Notice that the square-root term can always be written in the form

$$
f\left(1+t^{2} \pm 2 t x\right)^{-1 / 2}, \quad x=\cos \theta,
$$

where the expressions for $f$ and $t$ are given in Table VI.

We also know by the definition of the Legendre polynomials [14] that

$$
\left(1+t^{2} \pm 2 t x\right)^{-1 / 2}=\sum_{L^{\prime}=0}^{\infty}(\mp)^{L^{\prime}} t^{L^{\prime}} P_{L^{\prime}}(x), \quad|t|<1 .
$$

Because of the selection rules used below, the condition $|t|<1$ is not mandatory. Using the addition theorem for $P_{L}(x)$ and the fact that

$$
\mathcal{Y}_{l_{1} l_{2}}^{L M}\left(\hat{\xi}_{1}, \hat{\xi}_{2}\right)=\sum_{m_{1}, m_{2}}\left\langle L M \mid l_{1} m_{1} l_{2} m_{2}\right\rangle Y_{l_{1} m_{1}}\left(\hat{\xi}_{1}\right) Y_{l_{2} m_{2}}\left(\hat{\xi}_{2}\right)
$$

we obtain, after some straightforward algebra, the expression 
TABLE VI. Expressions for $t$ and $f$ appearing in Eq. (A2).

\begin{tabular}{lcccc}
\hline & \multicolumn{2}{c}{$(+)$ sign } & & $(-)$ sign \\
\cline { 2 - 5 }$\alpha$ interval & $f$ & 1 & & $f$ \\
\hline $0 \leq \alpha \leq \alpha_{-}$ & $\left(\eta_{+} \cos \alpha\right)^{-1}$ & $\tan \alpha / \eta_{+}$ & $\left(\eta_{-} \cos \alpha\right)^{-1}$ & $\tan \alpha / \eta_{-}$ \\
$\alpha \leq \alpha \leq \alpha_{+}$ & $\left(\eta_{+} \cos \alpha\right)^{-1}$ & $\tan \alpha / \eta_{+}$ & $(\sin \alpha)^{-1}$ & $\begin{array}{c}\eta_{-} \cot \alpha \\
\alpha_{+} \leq \alpha \leq \pi / 2\end{array}$ \\
\hline
\end{tabular}

$$
\langle\hat{v}\rangle=\left(\frac{\mu_{1}}{\mu}\right)^{1 / 2} \frac{q_{1} q_{2}}{\sin \alpha} \delta_{l_{1} l_{l}^{\prime} l_{2} l_{2}^{\prime}}+\frac{m_{1}}{\sqrt{\mu \mu_{1}}} q_{1} q_{3} \Delta_{l_{1} l_{1}^{\prime} l_{2} l_{2}^{\prime}}^{+}(\alpha)+\frac{m_{2}}{\sqrt{\mu \mu_{2}}} q_{2} q_{3} \Delta_{l_{1} l_{1}^{\prime} l_{2} l_{2}^{\prime}}^{-}(\alpha),
$$

where

$$
\Delta_{l_{1} l_{1}^{\prime} l_{2} l_{2}^{\prime}}^{ \pm}(\alpha)=\sum_{L^{\prime}}( \pm)^{L^{\prime}} C_{l_{1} l_{1}^{\prime} l_{2} l_{2}^{\prime}}^{L L_{2}^{\prime}} D_{L^{\prime}}^{ \pm}(\alpha)
$$

and

$$
\mathcal{C}_{l_{1} l_{1}^{\prime} l_{2} l_{2}^{\prime}}^{L L^{\prime}}=(-)^{L+L^{\prime}}\left[\left(2 l_{1}+1\right)\left(2 l_{1}^{\prime}+1\right)\left(2 l_{2}+1\right)\left(2 l_{2}^{\prime}+1\right)\right]^{1 / 2}\left(\begin{array}{ccc}
l_{1} & l_{1}^{\prime} & L^{\prime} \\
0 & 0 & 0
\end{array}\right)\left(\begin{array}{ccc}
l_{2} & l_{2}^{\prime} & L^{\prime} \\
0 & 0 & 0
\end{array}\right)\left\{\begin{array}{lll}
l_{1} & l_{2} & L \\
l_{2}^{\prime} & l_{1}^{\prime} & L^{\prime}
\end{array}\right\},
$$

where the usual $3-j$ and $6-j$ symbols appear. The $D_{L}^{ \pm}$terms are given by

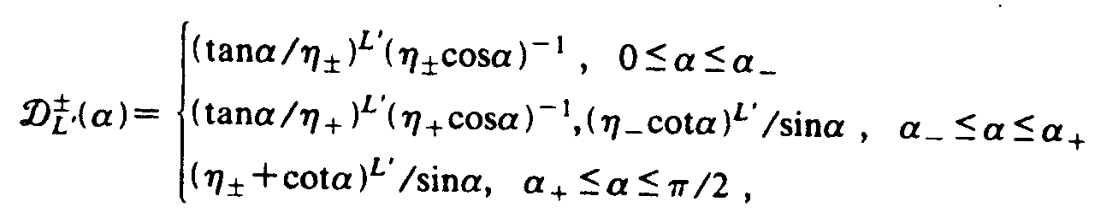
where $\tan \alpha_{ \pm}=\eta_{ \pm}$. In terms of the variable $z, D_{L^{\prime}}^{ \pm}(z)$ can easily be obtained by noticing that $\tan \alpha=2 z /\left(1-z^{2}\right)$,
$\cot \alpha=\left(1-z^{2}\right) / 2 z$, ( $\left.\sin \alpha\right)^{-1}=\left(1+z^{2}\right) / 2 z$, and $(\cos \alpha)^{-1}=\left(1+z^{2}\right) /\left(1-z^{2}\right)$.

[1] Z. Chen and C. D. Lin, Phys. Rev. A 42, 18 (1990).

[2] Yu. A. Simonov, in Proceedings of the International Sym. posium on the Present Status and Novel Developments in the Nuclear Many-body Problems, Rome, 1972, edited by F. Calogero and C. Ciofi degli Atti (Editrice Compositori, Bologna, 1973), p. 527; L. M. Delves, Nucl. Phys. 9, 391 (1959); Nucl. Phys. 20, 268 (1962); W. Zickendrath, Ann. Phys. (N.Y.) 35, 18 (1965); Phys. Rev. 159, 1448 (1967); F. T. Smith, ibid. 120, 1058 (1960).

[3] M. I. Haftel and V. B. Mandelzweig, Phys. Rev. A 38, 5995 (1988)

[4] J. H. Macek, J. Phys. B 1, 831 (1968).

[5] J. H. Macek, Phys. Rev. A 31, 2162 (1985); J. Macek and K. A. Jerjian, ibid. 33, 233 (1986).

[6] V. P. Brito, H. T. Coelho, and T. K. Das, Phys. Rev. A 40, 3346 (1989); S. K. Adhikari, V. P. Brito, H. T. Coelho, and T. K. Das, Nuovo Cimento B (to be published).

[7] J. E. Hornos, S. W. MacDowell, and C. D. Caldwell, Phys. Rev. A 33, 2212 (1986).

[8] H. T. Coelho, T. K. Das, and M. R. Robilotta, Phys. Rev. C 28, 1812 (1983); T. K. Das, H. T. Coelho, and M. Fabre de la Ripelle, Phys. Rev. C 26, 2281 (1982).
[9] C. H. Greene, Phys. Rev. A 23, 661 (1981).

[10] U. Fano, Rep. Prog. Phys. 46, 97 (1983).

[11] C. D. Lin and X. Liu, Phys. Rev. A 37, 2749 (1988).

[12] H. T. Coelho and J. E. Hornos, Phys. Rev. A 43, 6379 (1991).

[13] Yu. F. Smirnov and K. V. Shitikova, Sov. J. Part. Nucl. 8(4), 344 (1977) [Fiz. Elem. Chastits At. Yadra 8, 847 (1977)].

[14] Handbook of Mathematical Functions, edited by $\mathbf{M}$. Abramowitz and I. A. Stegun, 3rd ed. (Dover, New York, 1965), Chap. 22.

[15] M. Suffczynski, W. Gorzkowski, and X. Kowalczyk, Phys. Lett. 24A, 453 (1967).

[16] T. Skettrup, M. Suffczynski, and W. Orzkowski, Phys. Rev. B 4, 512 (1971).

[17] J. J. de Groote, J. E. Hornos, H. T. Coelho, and C. D. Caldwell, Phys. Rev. B 46, 2102 (1992).

[18] Ya. Zel'dovich and S. S. Gershein, Sov. Phys. Usp. 3, 593 (1961) [Usp. Fiz. Nauk 71, 581 (1960)].

[19] S. Hara and T. Ishihara, Phys. Rev. A 40, 4232 (1989).

[20] J. E. Pollard, D. J. Trevor, J. E. Reult, Y. T. Lee, and D. A. Shirley, J. Chem. Phys. 77 (1), 34 (1982). 


\section{Bibliografia}




\section{Referências}

[1] U. Fano, Rep. Prog. Phys. 46, 97 (1983).

[2] J. H. Macek, J. Phys. B 1, 831 (1968).

[3] 'T.K. Das, H.T. Coelho, and M. Frabe de la Ripelle, Phys.Rev. C 26, 2281 (1982)

[4] H.T. Coelho, T.K. Das, and M.R. Robilota, Phys.Rev. (: 28, 1812 (1983)

[5] U. Fano, Physics Today 29, $N_{0} .9,32$ (1976).

[6] C.H. Greene, Phys. Rev. A 23, 661 (1981).

[7] C.D. Lin, Phys. Rev A 10, 1986 (1974)

[8] Z. Chem and C.D. Lin, Phys. Rev. A 42, 18 (1990)

[9] C.D. Lin and X. Liu, Phys. Rev. A 37, 2749 (1988).

[10] J. E. Hornos, S. W. MacDowell, and C. D. Caldwell, Phys. Rev. A 33, 2212 (1986).

[11] H. T. Coelho and J. E. Hornos, Phys. Rev. A 43, 6379 (1991).

[12] J. J. De Groote, J. E. Hornos, H. T. Coelho, and C. D. Caldwell, Phys. Rev. B 46, 2102 (1992).

[13] J. J. De Groote, M. Masili, and J. E. Hornos, Analytical functions for the calculation of hyperspherical potential curves of atomic and molecular systems (em preparação).

[14] M. Masili, J. J. De Groote, and J. E. Hornos, Non-adiabatic coupling in the hyperspherical approach: precise wave functions for llelium (cm preparaça)).

[15] J. H. Bartlett, Phys. Rev. 51, 661 (1937).

[16] V. A. Fock, Izv. Akad. Nauk SSSR, Ser. Fiz. 18, 161 (1954).

[17] E. M. Ermolaev and G. B. Sochilin, Dokl. Akad. Nauk SSSR 155, 1050 (1964).

[18] K. Frankowski and C. L. Pekeris, Phys. Rev. 146, 16 (1966).

[19] H.T. Coelho, J.J. De Groote and J.E. Hornos, Phys. Rev. A 46, 5443 (1992)

[20] M. Masili, Dissertação de Mestrado, Acoplamentos Não Adiabáticos pelo Método Hiperesférico, USP (1993)

[21] Handbook of Mathematical Functions, edited by M. Abramowilz and I. A. Stegun, 3rd ed. (Dover, New York, 1965)

[22] C. L. Pekeris, Phys. Rev. 115, 1216 (1959).

[23] J.J. Hopfield, Proccedings of the seventh international Confernce on the Physics of Semiconductor, Paris, 1964 (Dundod, Paris, 1964, P.725) 
[24] R.R. Sharma and S. Rodriguez, Phys. Rev. 153, 823 (1967)

[25] M. Suffczynski, W. Gorzkowski, and Kowalczyk, Phys. Lett. 24A, 453 (1967)

[26] T. Skettrup, M. Suffczynski, and W. Orzkowski, Phys. Rev. B 4, 512 (1971)

[27] Tsin-Fu Jiang, Solid State Commun. 74, 899 (1990)

[28] J:A. Wheeler, Ann. NY Acad. Sci. 48, 219 (1946)

[29] A.P. Mills, Jr., Phys. Rev. Lett. 46, 717 (1981)

[30]. J.M. Rost and D. Wintgen, Phys. Rev. Lett. 69, 2499 (1992)

[31] P. Petelenz and J.H. Smith, Phys. Rev. A 36, 5125 (1987)

[32] F.C. Frank, Nature 160, 525 (1947)

[33] A.D. Sakharov, Report of the Physics Institute, Academy of Sciences, (1948).

[34] Alvarez et al., Phys. Rev. 105, 1127 (1957) 
[24] R.R. Sharma and S. Rodriguez, Phys. Rev. 153, 823 (1967)

[25] M. Suffczynski, W. Gorzkowski, and Kowalczyk, Phys. Lett. 24A, 453 (1967)

[26] T. Skettrup, M. Suffczynski, and W. Orzkowski, Phys. Rev. B 4, 512 (1971)

[27] Tsin-Fu Jiang, Solid State Commun. 74, 899 (1990)

[28] J.A. Wheeler, Ann. NY Acad. Sci. 48, 219 (1946)

[29] A.P. Mills, Jr., Phys. Rev. Lett. 46, 717 (1981)

[30] J.M. Rost and D. Wintgen, Phys. Rev. Lett. 69, 2499 (1992)

[31] P. Petelenz and J.H. Smith, Phys. Rev. A 36, 5125 (1987)

[32] F.C. Frank, Nature 160, 525 (1947)

[33] A.D. Sakharov, Report of the Physics Institute, Academy of Sciences, (1948).

[34] Alvarez et al., Phys. Rev. 105, 1127 (1957) 